\title{
ERROR ANALYSIS OF A FULLY DISCRETE FINITE ELEMENT METHOD FOR VARIABLE DENSITY INCOMPRESSIBLE FLOWS IN TWO DIMENSIONS
}

\author{
Wentao CaI ${ }^{1}$, BuYAng $\mathrm{LI}^{2, *}$ AND Ying $\mathrm{LI}^{3}$
}

\begin{abstract}
An error estimate is presented for a fully discrete, linearized and stabilized finite element method for solving the coupled system of nonlinear hyperbolic and parabolic equations describing incompressible flow with variable density in a two-dimensional convex polygon. In particular, the error of the numerical solution is split into the temporal and spatial components, separately. The temporal error is estimated by applying discrete maximal $L^{p}$-regularity of time-dependent Stokes equations, and the spatial error is estimated by using energy techniques based on the uniform regularity of the solutions given by semi-discretization in time.
\end{abstract}

Mathematics Subject Classification. 65N30, 76M05.

Received November 18, 2018. Accepted April 17, 2020.

\section{INTRODUCTION}

The time-dependent incompressible flow with variable density is governed by the partial differential equations (PDEs)

$$
\begin{aligned}
\partial_{t} \rho+\nabla \cdot(\rho \mathbf{u}) & =0 \\
\rho \partial_{t} \mathbf{u}+\rho \mathbf{u} \cdot \nabla \mathbf{u}+\nabla p-\mu \Delta \mathbf{u} & =0 \\
\nabla \cdot \mathbf{u} & =0
\end{aligned}
$$

where $\rho, \mathbf{u}$ and $p$ denote the density, velocity and pressure of the fluid, respectively, and $\mu>0$ the viscosity constant. We consider (1.1)-(1.3) in a convex polygon $\Omega \subset \mathbb{R}^{2}$ up to a given time $T$, with the following boundary and initial conditions:

$$
\begin{aligned}
& \mathbf{u}=0 \quad \text { on } \quad \partial \Omega \times[0, T], \\
& \rho=\rho^{0} \quad \text { and } \quad \mathbf{u}=\mathbf{u}^{0} \quad \text { at } \quad t=0,
\end{aligned}
$$

where $\rho^{0}$ and $\mathbf{u}^{0}$ are given functions, and $\partial \Omega$ the boundary of the domain $\Omega$. For given smooth initial data $\rho^{0}$ and $\mathbf{u}^{0}$ with positive density, i.e.,

$$
\min _{x \in \Omega} \rho^{0}(x)>0
$$

Keywords and phrases. Navier-Stokes, variable density, finite element, convergence, maximal $L^{p}$-regularity.

1 Department of Mathematics, School of Sciences, Hangzhou Dianzi University, Hangzhou 310018, P.R. China.

2 Department of Applied Mathematics, The Hong Kong Polytechnic University, Kowloon, Hong Kong.

3 School of Computer Engineering and Science, Shanghai University, Shanghai 2000444, P.R. China.

${ }^{*}$ Corresponding author: buyang.li@polyu.edu.hk 
the existence and uniqueness of smooth solutions of (1.1)-(1.4) have been proved in [14,32,44]. In particular, this hyperbolic-parabolic system does not generate shock wave (at least in 2D).

Many efficient numerical methods have been developed for solving (1.1)-(1.4), including the projection finite element methods (FEMs) [5,7,23,40,46], the fractional-step methods with pressure Poisson equation [24,25], the BDF stepping methods [41], the finite volume method [11], and the discontinuous Galerkin (DG) method [42]. However, there are very few work on rigorous error analysis of the numerical methods.

As mentioned in [46], the variable density introduces considerable difficulties for the analysis of the accuracy of the numerical solutions, mainly due to the strong nonlinearities and the coupling of the equations. The main difficulty in the error analysis of such nonlinear problems is to prove certain boundedness of the numerical solutions uniformly with respect to the step size and mesh size, while the presence of the hyperbolic density equation increases the difficulty: it requires the numerical solution $\mathbf{u}_{h}^{n}$ of the velocity equation to be bounded in a very strong norm, i.e.,

$$
\tau \sum_{n=1}^{N}\left\|\nabla \cdot \mathbf{u}_{h}^{n}\right\|_{L^{\infty}(\Omega)} \leq c,
$$

where $c$ should be independent of the temporal step size $\tau$ and spatial mesh size $h$.

An error estimate for the velocity equations (1.2) and (1.3) was presented in [26] for the numerical methods developed in $[24,25]$ by assuming that the numerical solutions $\rho_{h}^{n}, n=1, \ldots, N$, of the density equation have the following properties (cf. [26], Conjectures in Rem. 4.2):

(A1) Positivity and boundedness: $c_{1} \leq \min _{x \in \bar{\Omega}} \rho_{h}^{n}(x) \leq \max _{x \in \bar{\Omega}} \rho_{h}^{n}(x) \leq c_{2}, n=1,2, \ldots, N$, where $c_{1}$ and $c_{2}$ are positive constants independent of the step size and mesh size.

(A2) Error estimate of $\rho_{h}^{n}$ in terms of $\mathbf{u}_{h}^{n}$ (numerical solution of the velocity equation):

$$
\begin{aligned}
& \tau \sum_{n=1}^{N}\left(\left\|\rho\left(\cdot, t_{n}\right)-\rho_{h}^{n}\right\|_{L^{2}(\Omega)}^{2}+\left\|\partial_{t} \rho\left(\cdot, t_{n}\right)-\frac{\rho_{h}^{n}-\rho_{h}^{n-1}}{\tau}\right\|_{H^{-1}(\Omega)}^{2}\right) \\
& \quad \leq c_{\epsilon}\left(\tau+h^{m}\right)^{2}+\tau \sum_{n=1}^{N}\left(c_{\epsilon}\left\|\mathbf{u}\left(\cdot, t_{n}\right)-\mathbf{u}_{h}^{n}\right\|_{L^{2}(\Omega)}^{2}+\epsilon\left\|\mathbf{u}\left(\cdot, t_{n}\right)-\mathbf{u}_{h}^{n}\right\|_{H^{1}(\Omega)}^{2}\right),
\end{aligned}
$$

where $\epsilon \in(0,1)$ can be arbitrarily small, and the constant $c_{\epsilon}$ depends on $\epsilon$.

However, a rigorous proof of (A1) and (A2) remains open. Thus a complete error estimate for the coupled system (1.1)-(1.4) is still missing.

The stability of the numerical solutions was investigated in $[23,40,46]$ for different discretizations of the stabilized equations

$$
\begin{aligned}
\partial_{t} \rho+\mathbf{u} \cdot \nabla \rho+\frac{1}{2} \rho \nabla \cdot \mathbf{u} & =0, \\
\rho \partial_{t} \mathbf{u}+\rho \mathbf{u} \cdot \nabla \mathbf{u}+\frac{1}{2} \partial_{t} \rho \mathbf{u}+\frac{1}{2} \nabla \cdot(\rho \mathbf{u}) \mathbf{u}-\Delta \mathbf{u}+\nabla p & =0, \\
\nabla \cdot \mathbf{u} & =0
\end{aligned}
$$

which are theoretically equivalent to (1.1)-(1.3) but preserve the energy conservation upon discretization with the FEMs. The stability property of numerical schemes does not imply error estimates, but can be used to prove the convergence of DG via a compactness argument [42] (without explicit order of convergence).

In this article, we present a complete error estimate for a linearized and stabilized FEM for solving the coupled system (1.1)-(1.4). The techniques include discrete maximal $L^{p}$-regularity of parabolic equations recently established in [30] and an error splitting technique developed in $[17,35,36]$.

The discrete maximal $L^{p}$-regularity, established in a series of articles $[6,18,30,33,34,38]$, is a mathematical tool for analysis of both time discretizations [3,4,31] and finite element spatial discretizations [37] of nonlinear 
parabolic equations. The technique used in this paper extends the result of $[3,4]$ to parabolic equations with a time-dependent coefficient in front of the time derivative (see Lem. 4.2). This extension is needed to treat the velocity equation in the presence of the variable density $\rho$ in front of the time derivative.

The Helmholtz-Weyl decomposition, also known as Hodge decomposition, decomposes a vector field into a sum of its divergence-free and curl-free parts. It was used for numerical analysis of many different partial differential equations, including the Maxwell equations [10], the Ginzburg-Landau equations [39], and the Navier-Stokes equations with constant density $[1,12,27,45,47-49]$. In the presence of a variable density, the term $\rho \partial_{t} \mathbf{u}$ is no long divergence-free, and thus the projection of (1.2) onto the divergence-free subspace is more complicated.

The rest of this paper is organized as follows. In the next section, we introduce the notations, numerical scheme and main theorem. We present an overview for the proof of the main theorem in Section 3 and present the details in Sections 4 and 5. Numerical results are presented in Section 6 to support the theoretical analysis.

\section{Notations AND MAin RESUlts}

\subsection{Function spaces}

For any integer $k \geq 0$ and real number $1 \leq p \leq \infty, W^{k, p}(\Omega)$ denotes the conventional Sobolev space of functions defined on the domain $\Omega$, with the abbreviations $H^{k}(\Omega)=W^{k, 2}(\Omega)$ and $L^{p}(\Omega)=W^{0, p}(\Omega)$, and

$$
L_{0}^{p}(\Omega)=\left\{q \in L^{p}(\Omega): \int_{\Omega} q \mathrm{~d} x=0\right\} .
$$

The space of continuous functions on $\bar{\Omega}$ is denoted by $C(\bar{\Omega})$, and the space of infinitely differentiable functions with compact support in $\Omega$ is denoted by $C_{0}^{\infty}(\Omega)$. The closure of $C_{0}^{\infty}(\Omega)$ in $W^{k, p}(\Omega)$ is denoted by $W_{0}^{k, p}(\Omega)$, with the abbreviation $H_{0}^{k}(\Omega)=W_{0}^{k, 2}(\Omega)$. The vector-valued extensions of these function spaces are denoted by

$$
\mathbf{W}^{k, p}(\Omega)=W^{k, p}(\Omega) \times W^{k, p}(\Omega), \quad \mathbf{W}_{0}^{k, p}(\Omega)=W_{0}^{k, p}(\Omega) \times W_{0}^{k, p}(\Omega),
$$

with the abbreviations $\mathbf{H}^{k}(\Omega)=\mathbf{W}^{k, 2}(\Omega)$ and $\mathbf{L}^{p}(\Omega)=\mathbf{W}^{0, p}(\Omega)$.

For the simplicity of notations, the inner products of both $L^{2}(\Omega)$ and $\mathbf{L}^{2}(\Omega)$ are denoted by $(\cdot, \cdot)$, namely,

$$
\begin{aligned}
(w, v) & =\int_{\Omega} u(x) v(x) \mathrm{d} x, \quad \forall w, v \in L^{2}(\Omega), \\
(\mathbf{w}, \mathbf{v}) & =\int_{\Omega} \mathbf{w}(x) \cdot \mathbf{v}(x) \mathrm{d} x, \quad \forall \mathbf{w}, \mathbf{v} \in \mathbf{L}^{2}(\Omega) .
\end{aligned}
$$

Similarly, the norms of both $W^{k, p}(\Omega)$ and $\mathbf{W}^{k, p}(\Omega)$ are denoted by $\|\cdot\|_{W^{k, p}}$.

Following the notations of [4] (also see [3]), for any given sequence of functions $v^{n} \in W^{k, q}(\Omega), n=1,2, \ldots, m$, we define the norm

$$
\left\|\left(v^{n}\right)_{n=1}^{m}\right\|_{L^{p}\left(W^{k, q}(\Omega)\right)}= \begin{cases}\left(\tau \sum_{n=1}^{m}\left\|v^{n}\right\|_{W^{k, q}(\Omega)}^{p}\right)^{\frac{1}{p}} & \text { if } \quad 1 \leq p<\infty, \\ \max _{1 \leq n \leq m}\left\|v^{n}\right\|_{W^{k, q}(\Omega)} & \text { if } \quad p=\infty .\end{cases}
$$

Similarly, for a function $v$ defined on $\Omega \times(0, T)$ we define the following Borchner norm:

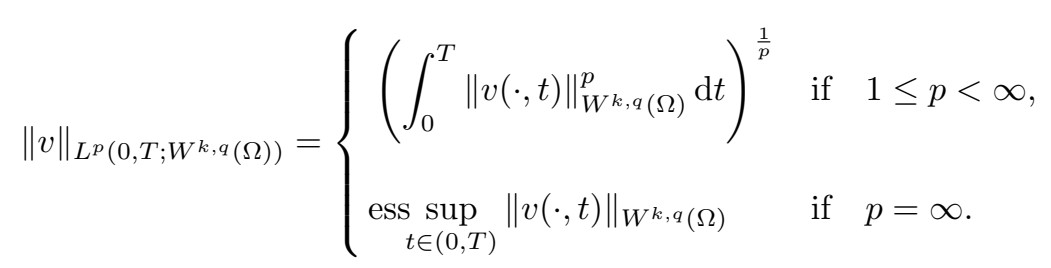




\subsection{Variational equations and stabilization}

We define the bilinear form

$$
B((\mathbf{u}, p),(\mathbf{v}, q)):=(\mu \nabla \mathbf{u}, \nabla \mathbf{v})-(p, \nabla \cdot \mathbf{v})+(\nabla \cdot \mathbf{u}, q), \quad \forall(\mathbf{u}, p),(\mathbf{v}, q) \in \mathbf{H}_{0}^{1}(\Omega) \times L_{0}^{2}(\Omega) .
$$

Note that if $(\rho, \mathbf{u}, p)$ is a solution of (1.1)-(1.3) then $(\rho, \mathbf{u}, p+c)$ is also a solution of (1.1)-(1.3), where $c$ can be an arbitrary constant. Besides, any sufficiently smooth solution of (1.1)-(1.3) satisfies (1.5)-(1.7) and thus the following variational equations:

$$
\begin{array}{r}
\left(\partial_{t} \rho, \varphi\right)+(\mathbf{u} \cdot \nabla \rho, \varphi)+\frac{1}{2}(\nabla \cdot \mathbf{u} \rho, \varphi)=0 \\
\left(\rho \partial_{t} \mathbf{u}+\rho \mathbf{u} \cdot \nabla \mathbf{u}, \mathbf{v}\right)+\frac{1}{2}\left(\partial_{t} \rho \mathbf{u}, \mathbf{v}\right)+\frac{1}{2}(\nabla \cdot(\rho \mathbf{u}) \mathbf{u}, \mathbf{v})+B((\mathbf{u}, p),(\mathbf{v}, q))=0
\end{array}
$$

where $\varphi \in L^{2}(\Omega)$ and $(\mathbf{v}, q) \in \mathbf{H}_{0}^{1}(\Omega) \times L_{0}^{2}(\Omega)$ are arbitrary test functions.

In the equations above, the extra terms $\frac{1}{2}(\nabla \cdot \mathbf{u} \rho, \varphi)$ and $\frac{1}{2}\left(\partial_{t} \rho \mathbf{u}, \mathbf{v}\right)+\frac{1}{2}(\nabla \cdot(\rho \mathbf{u}) \mathbf{u}, \mathbf{v})$ vanish due to (1.3) and (1.1), respectively. These extra terms will stabilize the finite element solutions to be defined in the next subsection.

\subsection{Spatial and temporal discretizations}

Let $\mathscr{T}_{h}$ be a quasi-uniform triangulation of the convex polygon $\Omega$ into triangles $\mathcal{T}_{j}, j=1, \ldots, M$, with mesh size $h=\max _{1 \leq j \leq M} \operatorname{diam}\left(\mathcal{T}_{j}\right)$. Let $P_{r}\left(\mathcal{T}_{j}\right)$ denote the space of polynomials of degree $\leq r$ on the triangle $\mathcal{T}_{j}$, and define the following finite element spaces:

$$
\begin{array}{ll}
\mathbf{X}_{h}^{r}=\left\{u_{h} \in H_{0}^{1}(\Omega)^{2}:\left.u_{h}\right|_{\mathcal{T}_{j}} \in P_{r}\left(\mathcal{T}_{j}\right)^{2},\right. & \left.\forall \mathcal{T}_{j} \in \mathscr{T}_{h}\right\}, \\
M_{h}^{r}=\left\{q_{h} \in H^{1}(\Omega):\left.q_{h}\right|_{\mathcal{T}_{j}} \in P_{r}\left(\mathcal{T}_{j}\right),\right. & \left.\forall \mathcal{T}_{j} \in \mathscr{T}_{h}\right\}, \\
\stackrel{\circ}{h}_{h}^{r}=\left\{q_{h} \in M_{h}^{r}: \int_{\Omega} q_{h}(x) \mathrm{d} x=0\right\} . &
\end{array}
$$

Consequently, $\mathbf{X}_{h}^{2} \times \stackrel{\circ}{M}_{h}^{1}$ is the lowest order Taylor-Hood finite element space, which satisfies the following inf-sup condition ( $c f$. [8], Thm. 4.1)

$$
\left\|q_{h}\right\|_{L^{2}(\Omega)} \leq c \sup _{\mathbf{v}_{h} \in \mathbf{X}_{h}^{2}} \frac{\left|\left(\nabla q_{h}, \mathbf{v}_{h}\right)\right|}{\left\|\nabla \mathbf{v}_{h}\right\|_{L^{2}(\Omega)}}, \quad \forall q_{h} \in \stackrel{\leftrightarrow}{M}_{h}^{1}
$$

We choose the finite element space $M_{h}^{2} \times \mathbf{X}_{h}^{2} \times \stackrel{\circ}{M}_{h}^{1}$ for the approximation of the solution $(\rho, \mathbf{u}, p)$.

For any given function $\varphi$, we define the truncated function

$$
\widehat{\varphi}=\max \left(\frac{1}{2} \min _{x \in \bar{\Omega}} \rho^{0}(x), \varphi\right) .
$$

Then $\widehat{\varphi} \geq \frac{1}{2} \min _{x \in \bar{\Omega}} \rho^{0}(x)>0$. Let $t_{n}=n \tau, n=0,1, \ldots, N$, be a uniform partition of the time interval $[0, T]$ with step size $\tau=T / N$. Based on the variational equations (2.4) and (2.5), we approximate the solution $\left(\rho\left(\cdot, t_{n}\right), \mathbf{u}\left(\cdot, t_{n}\right), p\left(\cdot, t_{n}\right)\right)$ by the finite element function $\left(\rho_{h}^{n}, \mathbf{u}_{h}^{n}, p_{h}^{n}\right) \in M_{h}^{2} \times \mathbf{X}_{h}^{2} \times \stackrel{M}{h}_{h}^{1}$, defined as the solution of the following equations:

$$
\begin{gathered}
\left(D_{\tau} \rho_{h}^{n}, \varphi_{h}\right)+\left(\mathbf{u}_{h}^{n-1} \cdot \nabla \rho_{h}^{n}, \varphi_{h}\right)+\frac{1}{2}\left(\nabla \cdot \mathbf{u}_{h}^{n-1} \rho_{h}^{n}, \varphi_{h}\right)=0 \\
\left(\hat{\rho}_{h}^{n-1} D_{\tau} \mathbf{u}_{h}^{n}, \mathbf{v}_{h}\right)+\frac{1}{2}\left(D_{\tau} \widehat{\rho}_{h}^{n} \mathbf{u}_{h}^{n}, \mathbf{v}_{h}\right)+\frac{1}{2}\left(\nabla \cdot\left(\rho_{h}^{n} \mathbf{u}_{h}^{n-1}\right) \mathbf{u}_{h}^{n}, \mathbf{v}_{h}\right) \\
+\left(\rho_{h}^{n} \mathbf{u}_{h}^{n-1} \cdot \nabla \mathbf{u}_{h}^{n}, \mathbf{v}_{h}\right)+B\left(\left(\mathbf{u}_{h}^{n}, p_{h}^{n}\right),\left(\mathbf{v}_{h}, q_{h}\right)\right)=0
\end{gathered}
$$


where $\left(\varphi_{h}, \mathbf{v}_{h}, q_{h}\right) \in M_{h}^{2} \times \mathbf{X}_{h}^{2} \times \stackrel{\circ}{M}_{h}^{1}$ are test functions, and $D_{\tau} \rho_{h}^{n}:=\left(\rho_{h}^{n}-\rho_{h}^{n-1}\right) / \tau$ denotes the backward Euler difference. The initial data $\rho_{h}^{0}$ and $\mathbf{u}_{h}^{0}$ are defined as

$$
\rho_{h}^{0}=\Pi_{h} \rho^{0} \quad \text { and } \quad \mathbf{u}_{h}^{0}=\Pi_{h} \mathbf{u}^{0}
$$

where $\Pi_{h}: C(\bar{\Omega}) \rightarrow M_{h}^{2}$ and $\Pi_{h}: \mathbf{C}(\bar{\Omega}) \rightarrow \mathbf{X}_{h}^{2}$ denote the scalar- and vector-valued Lagrange interpolation operators, respectively.

If $\left(\rho_{h}^{k}, \mathbf{u}_{h}^{k}\right)$ are given for $k=0, \ldots, n-1$, then $\left(\rho_{h}^{n}, \mathbf{u}_{h}^{n}\right)$ can be solved in the following order:

(1) $\rho_{h}^{n}$ can be solved from $(2.11)$;

(2) $\widehat{\rho}_{h}^{n-1}$ and $\widehat{\rho}_{h}^{n}$ can be defined by (2.10);

(3) $\left(\mathbf{u}_{h}^{n}, p_{h}^{n}\right)$ can be solved from (2.12).

Remark 2.1. The stabilization terms $\frac{1}{2}\left(\nabla \cdot \mathbf{u}_{h}^{n-1} \rho_{h}^{n}, \varphi_{h}\right)$ and $\frac{1}{2}\left(D_{\tau} \widehat{\rho}_{h}^{n} \mathbf{u}_{h}^{n}, \mathbf{v}_{h}\right)+\frac{1}{2}\left(\nabla \cdot\left(\rho_{h}^{n} \mathbf{u}_{h}^{n-1}\right) \mathbf{u}_{h}^{n}, \mathbf{v}_{h}\right)$ in the numerical scheme (2.11) and (2.12) guarantees that the method is unconditionally stable, i.e., substituting $\varphi_{h}=\rho_{h}^{n}$ and $\mathbf{v}_{h}=\mathbf{u}_{h}^{n}$ into (2.11) and (2.12) yields the following energy equalities:

$$
\begin{aligned}
\left\|\rho_{h}^{n}\right\|^{2} & =\left\|\rho_{h}^{n-1}\right\|^{2}, \\
\int_{\Omega} \frac{1}{2} \widehat{\rho}_{h}^{n}\left|\mathbf{u}_{h}^{n}\right|^{2} \mathrm{~d} x+\tau \mu\left\|\nabla \mathbf{u}_{h}^{n}\right\|^{2} & =\int_{\Omega} \frac{1}{2} \widehat{\rho}_{h}^{n-1}\left|\mathbf{u}_{h}^{n-1}\right|^{2} \mathrm{~d} x .
\end{aligned}
$$

Since $\widehat{\rho}_{h}^{n}$ and $\widehat{\rho}_{h}^{n-1}$ are bounded from both below and above due to the truncation in (2.10), the two equalities above prove that both $\rho_{h}^{n}$ and $\mathbf{u}_{h}^{n}$ are bounded in the $L^{2}$ norm for all time levels without any restriction on the time stepsize $\tau$ and spatial mesh size $h$.

\subsection{Convergence of the numerical solutions}

In this work, we assume that the initial density $\rho^{0}(x)$ is positive (no vacuum) and that problem (1.1)-(1.4) has a sufficiently smooth solution, i.e.,

$$
\begin{aligned}
& 0<\min _{x \in \bar{\Omega}} \rho^{0}(x) \leq \max _{x \in \bar{\Omega}} \rho^{0}(x)<\infty, \\
& \rho \in C\left([0, T] ; H^{3}(\Omega) \cap W^{2, \infty}(\Omega)\right) \cap C^{2}\left([0, T] ; H^{2}(\Omega)\right), \\
& \mathbf{u} \in C\left([0, T] ; \mathbf{H}^{3}(\Omega)\right) \cap C^{1}\left([0, T] ; \mathbf{W}^{2, q}(\Omega)\right) \cap C^{2}\left([0, T] ; \mathbf{L}^{\infty}(\Omega)\right) \quad \text { for some } q>2, \\
& p \in C\left([0, T] ; H^{2}(\Omega)\right),
\end{aligned}
$$

and investigate the convergence of the numerical solutions defined in Section 2.3. In particular, we prove the following theorem.

Theorem 2.2. Suppose that the solution of (1.1)-(1.4) is sufficiently smooth, satisfying (2.14) and (2.15). Then the discrete problem (2.11)-(2.13) has a unique finite element solution $\left(\rho_{h}^{n}, \mathbf{u}_{h}^{n}, p_{h}^{n}\right) \in M_{h}^{2} \times \mathbf{X}_{h}^{2} \times M_{h}^{1}$, $n=1,2, \ldots, N$. Moreover, there exist positive constants $\tau_{*}$ and $h_{*}$ such that for $\tau \leq \tau_{*}$ and $h \leq h_{*}$ the following error estimates hold:

$$
\begin{array}{r}
\max _{1 \leq n \leq N}\left(\left\|\rho\left(\cdot, t_{n}\right)-\rho_{h}^{n}\right\|_{L^{2}}+\left\|\mathbf{u}\left(\cdot, t_{n}\right)-\mathbf{u}_{h}^{n}\right\|_{L^{2}}\right) \leq c\left(\tau+h^{2}\right), \\
\left(\tau \sum_{n=1}^{N}\left\|p\left(\cdot, t_{n}\right)-p_{h}^{n}\right\|_{L^{2}}^{2}\right)^{\frac{1}{2}} \leq c \sqrt{\tau+h^{2}},
\end{array}
$$

where $c$ is some positive constant independent of the temporal step size $\tau$ and spatial mesh size $h$ (possibly dependent on $T$ ). 
Remark 2.3. For the Navier-Stokes equations with constant density, the finite element solutions with the Taylor-Hood elements should have 3rd-order convergence. However, for the model with variable density, finite element solutions of the scalar hyperbolic density equation generally have lower-order convergence ( $c f$. [15], Rem. 3.14, 2nd-order or 2.5th-order convergence, depending on the stabilization techniques), which further polluted the order of convergence of the velocity $\mathbf{u}_{h}^{n}$ through the coupling of the equations.

Remark 2.4. The truncation $\widehat{\rho}_{h}^{n}$ in (2.12) is used only to guarantee the existence and uniqueness of finite element solutions for large step size $\tau$ and mesh size $h$. For sufficiently small $\tau$ and $h$, we have $\hat{\rho}_{h}^{n}=\rho_{h}^{n}$ (see the explanation in Sect. 5.3). Thus the truncation operation does not play a role in deriving the error estimates (2.16) and (2.17).

Remark 2.5. On the one hand, we have assumed that the solution is sufficiently smooth and investigate the convergence of numerical solutions. In particular, the $H^{3}$ regularity of solution is essential (due to the hyperbolic equation of $\rho$ ) in our analysis to obtain second-order convergence for the numerical solutions. On the other hand, we have assumed that the domain is convex polygonal in order to avoid approximating the boundary by piecewise linear lines (or quadratic curves), which leads to new errors that will make analysis more complicated. It is known that the solution can have $H^{2}$ regularity when the initial value is sufficiently smooth in a convex polygon such as rectangle. Whether the solution can have $H^{3}$ regularity in a convex polygon is not clear in general. In some special cases, the solution can have $H^{3}$ regularity if there are additional source terms in the equation (as shown in the numerical example of Sect. 6), which will not affect the error analysis in this paper.

Alternatively, if we assume that the domain is smooth and convex (instead of convex polygonal), then the solution can be sufficiently smooth as assumed in (2.14). In this case, the triangulated domain $\Omega_{h}$ is not equal to $\Omega$. For a triangle $\mathcal{T}_{j}$ with two vertices on the boundary, we can define $\widetilde{\mathcal{T}}_{j}$ to be the extension of $\mathcal{T}_{j}$ to a curved triangle which fits the boundary $\partial \Omega$ exactly. For a triangle $\mathcal{T}_{j}$ with at most one vertex on the boundary, we simply denote $\widetilde{\mathcal{T}}_{j}=\mathcal{T}_{j}$. We assume that a diffeomorphic map from $\mathcal{G}_{j}: \mathcal{T}_{j} \rightarrow \widetilde{\mathcal{T}}_{j}$ is known, satisfying

$$
\left\|\nabla^{l} \mathcal{G}_{j}\right\|_{L^{\infty}\left(\mathcal{T}_{j}\right)} \leq C \quad \text { and } \quad\left\|\nabla^{l} \mathcal{G}_{j}^{-1}\right\|_{L^{\infty}\left(\widetilde{\mathcal{T}}_{j}\right)} \leq C, \quad l=1, \ldots, 3 .
$$

Such a map exists if the domain $\Omega$ is convex and the boundary $\partial \Omega$ is sufficiently smooth. We denote by $\mathcal{G}: \Omega_{h} \rightarrow \Omega$ the corresponding global map such that $\left.\mathcal{G}\right|_{\mathcal{I}_{j}}=\mathcal{G}_{j}$. Then the finite element spaces

$$
\begin{array}{ll}
\mathbf{X}_{h}^{r}=\left\{u_{h} \in H_{0}^{1}(\Omega)^{2}:\left.\left(u_{h} \circ \mathcal{G}\right)\right|_{\mathcal{T}_{j}} \in P_{r}\left(\mathcal{T}_{j}\right)^{2},\right. & \left.\forall \mathcal{T}_{j} \in \mathscr{T}_{h}\right\} \\
M_{h}^{r}=\left\{q_{h} \in H^{1}(\Omega):\left.\left(q_{h} \circ \mathcal{G}\right)\right|_{\mathcal{I}_{j}} \in P_{r}\left(\mathcal{T}_{j}\right),\right. & \left.\forall \mathcal{T}_{j} \in \mathscr{T}_{h}\right\} \\
\stackrel{\circ}{h}_{h}^{r}=\left\{q_{h} \in M_{h}^{r}: \int_{\Omega} q_{h}(x) \mathrm{d} x=0\right\} &
\end{array}
$$

have the same approximation properties (and satisfies the inf-sup condition) as the standard finite element spaces on a convex polygon. As a result, the error analysis presented in this paper also holds for such special finite elements in a smooth and convex domain.

To prove Theorem 2.2, we investigate the temporal and spatial discretizations in Sections 4 and 5, separately. Throughout this paper, we denote by $c$ a generic positive constant and $\epsilon$ is a generic small positive constant, both are independent of $n, h$ and $\tau$, possibly different at each occurrence.

\section{An OVERVIEW FOR THE PROOF OF THEOREM 2.2}

In this Section, we present an overview for the proof of Theorem 2.2 for the readers' convenience. The proof consists of analysis of temporal and spatial discretizations, respectively, which we briefly introduce below. The details are presented in Sections 4 and 5. 


\subsection{Analysis of temporal discretization}

We introduce a semi-discrete problem

$$
\begin{aligned}
D_{\tau} \rho_{\tau}^{n}+\mathbf{u}_{\tau}^{n-1} \cdot \nabla \rho_{\tau}^{n} & =0, \\
\rho_{\tau}^{n-1} D_{\tau} \mathbf{u}_{\tau}^{n}+\rho_{\tau}^{n} \mathbf{u}_{\tau}^{n-1} \cdot \nabla \mathbf{u}_{\tau}^{n}+\nabla p_{\tau}^{n}-\mu \Delta \mathbf{u}_{\tau}^{n} & =0, \\
\nabla \cdot \mathbf{u}_{\tau}^{n} & =0,
\end{aligned}
$$

with the boundary and initial conditions

$$
\begin{array}{rlll}
\mathbf{u}_{\tau}^{n}=0 & \text { on } & & \partial \Omega, \quad n=1,2, \ldots, N, \\
\rho_{\tau}^{0}=\rho^{0} & \text { and } & \mathbf{u}_{\tau}^{0}=\mathbf{u}^{0} .
\end{array}
$$

Then the fully discrete solution defined by (2.11) and (2.12) can be viewed as the finite element solution of (3.1)-(3.4). The temporal discretization errors are denoted by

$$
e_{\rho}^{n}:=\rho^{n}-\rho_{\tau}^{n}, \quad \mathbf{e}_{\mathbf{u}}^{n}:=\mathbf{u}^{n}-\mathbf{u}_{\tau}^{n} \quad \text { and } \quad e_{p}^{n}:=p^{n}-p_{\tau}^{n},
$$

where

$$
\rho^{n}=\rho\left(\cdot, t_{n}\right), \quad \mathbf{u}^{n}=\mathbf{u}\left(\cdot, t_{n}\right), \quad p^{n}=p\left(\cdot, t_{n}\right) .
$$

In Section 4, we prove the following estimates for the temporal discretization errors, together with some regularity estimates for $\rho_{\tau}^{n}, \mathbf{u}_{\tau}^{n}$ and $p_{\tau}^{n}$ uniformly with respect to the step size $\tau$.

Proposition 3.1. Under the assumptions of Theorem 2.2, there exists a positive constant $\tau_{0}$ such that when $\tau \leq \tau_{0}$, the time-discrete system (3.1)-(3.4) has a unique solution $\left(\rho_{\tau}, \mathbf{u}_{\tau}, p_{\tau}\right)$ satisfying the maximum principle

$$
\min _{x \in \bar{\Omega}} \rho^{0}(x) \leq \rho_{\tau}^{n}(x) \leq \max _{x \in \bar{\Omega}} \rho^{0}(x), \quad \forall x \in \Omega, \quad n=1, \ldots, N,
$$

the error estimates

$$
\begin{gathered}
\max _{1 \leq n \leq N}\left(\left\|e_{\rho}^{n}\right\|_{H^{2}}+\left\|\mathbf{e}_{\mathbf{u}}^{n}\right\|_{H^{1}}+\left\|\mathbf{e}_{\mathbf{u}}^{n}\right\|_{L^{\infty}}\right) \leq c \tau, \\
\sum_{n=1}^{N} \tau\left(\left\|D_{\tau} \mathbf{e}_{\mathbf{u}}^{n}\right\|_{L^{2}}^{2}+\left\|\mathbf{e}_{\mathbf{u}}^{n}\right\|_{H^{2}}^{2}+\left\|e_{p}^{n}\right\|_{H^{1}}^{2}\right) \leq c \tau^{2},
\end{gathered}
$$

and the following regularity estimates:

$$
\begin{array}{r}
\max _{0 \leq n \leq N}\left(\left\|\mathbf{u}_{\tau}^{n}\right\|_{H^{2}}+\left\|\mathbf{u}_{\tau}^{n}\right\|_{W^{1, \infty}}+\left\|p_{\tau}^{n}\right\|_{H^{1}}+\left\|\rho_{\tau}^{n}\right\|_{H^{2}}+\left\|\rho_{\tau}^{n}\right\|_{W^{1, \infty}}+\left\|D_{\tau} \mathbf{u}_{\tau}^{n}\right\|_{L^{\infty}}\right) \leq c, \\
\sum_{n=1}^{N} \tau\left(\left\|D_{\tau} \mathbf{u}_{\tau}^{n}\right\|_{H^{2}}^{2}+\left\|D_{\tau} p_{\tau}^{n}\right\|_{H^{1}}^{2}\right) \leq c
\end{array}
$$

where the constant $c$ is independent of the step size $\tau$.

\subsection{Analysis of spatial discretization}

Proposition 3.1 gives the $H^{2}$ and $W^{1, \infty}$ regularity of the time-discrete solutions $\rho_{\tau}^{n}$ and $\mathbf{u}_{\tau}^{n}$ uniformly with respect to the step size $\tau$. By using such uniform regularity of the time-discrete solutions, the following error estimates for the fully discrete finite element solutions will be proved in Section 5. 
Proposition 3.2. Under the assumption of Theorem 2.2, there exist positive constants $\tau_{*} \leq \tau_{0}$ and $h_{*}$ such that the fully discrete solutions given by (2.11) and (2.12) satisfy the following estimates when $\tau \leq \tau_{*}$ and $h \leq h_{*}$ :

$$
\begin{aligned}
& \max _{1 \leq n \leq N}\left(\left\|\mathbf{u}_{\tau}^{n}-\mathbf{u}_{h}^{n}\right\|_{L^{2}}+\left\|\rho_{\tau}^{n}-\rho_{h}^{n}\right\|_{L^{2}}\right)+h\left(\tau \sum_{n=1}^{N}\left\|p_{\tau}^{n}-p_{h}^{n}\right\|_{L^{2}}^{2}\right)^{\frac{1}{2}}+\left(\tau \sum_{n=1}^{N}\left\|\mathbf{u}_{\tau}^{n}-\mathbf{u}_{h}^{n}\right\|_{H^{1}}^{2}\right)^{\frac{1}{2}} \\
& \quad \leq \operatorname{ch} \sqrt{\tau+h^{2}} .
\end{aligned}
$$

Propositions 3.1 and 3.2 together show that, in the case $\tau \leq \tau_{*}$ and $h \leq h_{*}$, the fully discrete finite element solutions obey the following error estimates:

$$
\begin{array}{r}
\max _{1 \leq n \leq N}\left(\left\|\mathbf{u}^{n}-\mathbf{u}_{h}^{n}\right\|_{L^{2}}+\left\|\rho^{n}-\rho_{h}^{n}\right\|_{L^{2}}\right) \leq c\left(\tau+h^{2}\right), \\
\left(\tau \sum_{n=1}^{N}\left\|p^{n}-p_{h}^{n}\right\|_{L^{2}}^{2}\right)^{\frac{1}{2}} \leq c \sqrt{\tau+h^{2}} .
\end{array}
$$

The proof of Theorem 2.2 is complete up to the proofs of Propositions 3.1 and 3.2, which are presented in Sections 4 and 5, respectively.

Remark 3.3. The factor $h$ (independent of $\tau$ ) in the energy error estimate (3.11) is the key to make the analysis go through. In fact, in the proof of Proposition 3.2, the uniform boundedness (uniform with respect to the step size $\tau$ and mesh size $h$ ) of

$$
\begin{array}{ll}
\left\|\rho_{\tau}^{n}\right\|_{W^{1, \infty}} \quad \text { and } \tau \sum_{n=1}^{N}\left\|\mathbf{u}_{\tau}^{n}\right\|_{W^{1, \infty}}^{2} & \text { (for the time-discrete solutions) } \\
\left\|\rho_{h}^{n}\right\|_{L^{\infty}}, \quad\left\|\mathbf{u}_{h}^{n}\right\|_{L^{\infty}} \quad \text { and } \tau \sum_{n=1}^{N}\left\|\mathbf{u}_{h}^{n}\right\|_{W^{1, \infty}}^{2} & \text { (for the numerical solutions) }
\end{array}
$$

are needed to control the strong nonlinearities in the coupling of the equations. The boundedness of (3.14) is proved in Proposition 3.1 by using discrete $L^{p}\left(W^{2, q}\right)$ estimates. The boundedness of (3.15) can be proved by applying the inverse inequality to the error estimates (3.11), e.g.,

$$
\begin{aligned}
\left\|P_{h} \rho_{\tau}^{n}-\rho_{h}^{n}\right\|_{L^{\infty}} & \leq c h^{-1}\left\|P_{h} \rho_{\tau}^{n}-\rho_{h}^{n}\right\|_{L^{2}} \\
& \leq c h^{-1}\left(\left\|P_{h} \rho_{\tau}^{n}-\rho_{\tau}^{n}\right\|_{L^{2}}+\left\|\rho_{\tau}^{n}-\rho_{h}^{n}\right\|_{L^{2}}\right) \\
& \leq c h^{-1}\left(c h^{2}\left\|\rho_{\tau}^{n}\right\|_{H^{2}}+c h \sqrt{\tau+h^{2}}\right) \\
& \leq c \sqrt{\tau+h^{2}},
\end{aligned}
$$

where $P_{h} \rho_{\tau}^{n}$ denotes the $L^{2}$ projection of $\rho_{\tau}^{n}$ onto the finite element space $M_{h}^{2}$. The last inequality requires the factor $h$ in the energy error estimate (3.11).

The proof of (3.11) requires (3.15) to hold at the $(n-1)$ th step, and implies (3.15) at the $n$th step via the inverse inequality, e.g., (3.16). Thus both can be proved by using mathematical induction.

In order to have the error estimate (3.11), we need $H^{2}$ and $W^{1, \infty}$ regularity of the time-discrete solutions $\rho_{\tau}^{n}$ and $\mathbf{u}_{\tau}^{n}$ uniformly with respect to the step size $\tau$. This is why we carry out the error estimates of $\rho_{\tau}^{n}$ and $\mathbf{u}_{\tau}^{n}$ in Proposition 3.1 in such strong norms, rather than the standard $L^{2}$ norm.

\section{Proof of Proposition 3.1}

The proof of Proposition 3.1 requires using the maximal $L^{p}$-regularity of time-discrete Stokes equations, which will be introduced in Section 4.2. The proof of Proposition 3.1 is presented in Section 4.3. 


\subsection{Helmholtz-Weyl decomposition}

We define the divergence-free subspace and curl-free subspace of $\mathbf{L}^{q}(\Omega)$ as follows (cf. [16], Eq. (III.1.4) and Thm. III.2.3):

$$
\begin{aligned}
\mathbf{L}_{\sigma}^{q}(\Omega) & =\left\{\mathbf{w} \in \mathbf{L}^{q}(\Omega): \nabla \cdot \mathbf{w}=0, \quad \mathbf{w} \cdot \mathbf{n}=0 \quad \text { on } \quad \partial \Omega\right\}, \\
\mathbf{L}_{\sigma}^{q}(\Omega)^{\perp} & =\left\{\nabla \phi: \phi \in W^{1, q}(\Omega)\right\},
\end{aligned}
$$

where $\mathbf{n}$ denotes the unit outward normal vector on the boundary $\partial \Omega$. We say that the Helmholtz decomposition exists on $\mathbf{L}^{q}(\Omega)$ if any function $\mathbf{v} \in \mathbf{L}^{q}(\Omega)$ has a unique decomposition

$$
\mathbf{v}=\mathbf{P}_{\operatorname{div}} \mathbf{v}+\nabla \phi
$$

with $\mathbf{P}_{\text {div }} \mathbf{v} \in \mathbf{L}_{\sigma}^{q}(\Omega)$ and $\nabla \phi \in \mathbf{L}_{\sigma}^{q}(\Omega)^{\perp}$ such that

$$
\left\|\mathbf{P}_{\mathrm{div}} \mathbf{v}\right\|_{L^{q}(\Omega)}+\|\nabla \phi\|_{L^{q}(\Omega)} \leq C\|\mathbf{v}\|_{L^{q}(\Omega)} .
$$

Here $\mathbf{P}_{\text {div }}: \mathbf{L}^{q}(\Omega) \rightarrow \mathbf{L}_{\sigma}^{q}(\Omega)$ is called the Helmholtz projection (if the Helmholtz decomposition exists).

In fact, the Helmholtz decomposition on $\mathbf{L}^{q}(\Omega)$ exists if and only if the Neumann problem

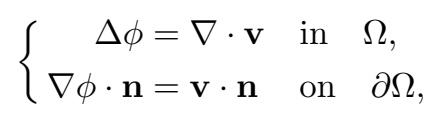

has a unique weak solution $\phi \in W^{1, q}(\Omega) \backslash \mathbb{R}$ satisfying

$$
\|\nabla \phi\|_{L^{q}(\Omega)} \leq C\|\mathbf{v}\|_{L^{q}(\Omega)}, \quad \forall \mathbf{v} \in \mathbf{L}^{q}(\Omega) .
$$

Then $\mathbf{P}_{\text {div }} \mathbf{v}=\mathbf{v}-\nabla \phi$ with $\phi$ given by (4.5).

In a smooth and convex domain, the $W^{1, q}$ solution of (4.5) exists (see [19], Thm. 1.2) and the Helmholtz decomposition (4.4) exists on $\mathbf{L}^{q}(\Omega)$ for all $1<q<\infty$. (see [19], Thm. 1.2 and [16], Lem. III.1.2). Therefore, the operator $\mathbf{P}_{\text {div }}$ is a bounded linear projection onto the divergence-free subspace $\mathbf{L}_{\sigma}^{q}(\Omega)$, satisfying

$$
\left\|\mathbf{P}_{\text {div }} \mathbf{v}\right\|_{L^{q}} \leq c\|\mathbf{v}\|_{L^{q}}, \quad \forall \mathbf{v} \in \mathbf{L}^{q}(\Omega) .
$$

In particular, we have

$$
\begin{aligned}
\mathbf{P}_{\mathrm{div}} \mathbf{v} & =\mathbf{v}, \quad \forall \mathbf{v} \in \mathbf{L}_{\sigma}^{q}(\Omega), \\
\mathbf{P}_{\mathrm{div}}(\nabla \phi) & =0, \quad \forall \phi \in W^{1, q}(\Omega) .
\end{aligned}
$$

\subsection{Discrete maximal $L^{p}$-regularity}

In [14], it was shown that the operator $\mathbf{P}_{\text {div }} \Delta$ has the maximal $L^{p}$-regularity on $\mathbf{L}_{\sigma}^{q}(\Omega)$, for $1<p, q<\infty$. Then Theorem 3.1 of [30] (for the initial term involving $v_{0}$, see Rem. 4.3) immediately implies the following result of discrete maximal $L^{p}$-regularity.

Lemma 4.1 (Discrete maximal $L^{p}$-regularity). Let $1 \leq m \leq N$, and let $a(x)$ be a function defined on $\Omega$ such that

(1) $\kappa_{0} \leq a(x) \leq \kappa_{1}$ for some positive constants $\kappa_{0}$ and $\kappa_{1}$;

(2) $\|a\|_{W^{1, \sigma}(\Omega)} \leq \kappa_{2}$ for some constants $\sigma>2$ and $\kappa_{2}>0$. 
Then the solutions of the equations

$$
a(x) D_{\tau} \mathbf{v}_{\tau}^{n}-\mathbf{P}_{\mathrm{div}} \Delta \mathbf{v}_{\tau}^{n}=\boldsymbol{f}^{n}, \quad n=1,2, \ldots, m,
$$

satisfy the following estimate:

$$
\begin{aligned}
& \left\|\left(D_{\tau} \mathbf{v}_{\tau}^{n}\right)_{n=1}^{m}\right\|_{L^{p}\left(L^{q}\right)}+\left\|\left(\mathbf{P}_{\mathrm{div}} \Delta \mathbf{v}_{\tau}^{n}\right)_{n=1}^{m}\right\|_{L^{p}\left(L^{q}\right)} \\
& \quad \leq c\left(\left\|\left(\boldsymbol{f}^{n}\right)_{n=1}^{m}\right\|_{L^{p}\left(L^{q}\right)}+\tau^{\frac{1}{p}-1}\left\|\mathbf{v}_{\tau}^{0}\right\|_{L^{q}}+\tau^{\frac{1}{p}}\left\|\mathbf{P}_{\mathrm{div}} \Delta \mathbf{v}_{\tau}^{0}\right\|_{L^{q}}\right), \quad \forall 1<p, q<\infty,
\end{aligned}
$$

where $c$ is independent of $\tau, m$ and $a(x)$ (but may depend on $\kappa_{0}, \kappa_{1}, \kappa_{2}, \sigma, p$ and $q$ ).

However, Lemma 4.1 cannot be directly applied to the physical equations considered in this paper, which requires analysis of the following type of equations:

$$
\rho_{\tau}^{n-1} D_{\tau} \mathbf{u}_{\tau}^{n}-\mathbf{P}_{\mathrm{div}} \Delta \mathbf{u}_{\tau}^{n}=\boldsymbol{f}^{n},
$$

with the coefficient $\rho_{\tau}^{n-1}$ depending on $n$. In the following lemma, we extend Lemma 4.1 to this setting by using the ideas of $[3,4]$.

Lemma 4.2 (Extension to time-dependent coefficients). Let $1 \leq m \leq N$, and let $a_{\tau}^{n-1}(x), n=1, \ldots, m$, be functions defined on $\Omega$ such that

(1) $\kappa_{0} \leq a_{\tau}^{n-1}(x) \leq \kappa_{1}$ for some positive constants $\kappa_{0}$ and $\kappa_{1}$;

(2) $\max _{1 \leq n \leq m}\left\|a_{\tau}^{n-1}\right\|_{W^{1, \sigma}(\Omega)} \leq \kappa_{2}$ for some constants $\sigma>2$ and $\kappa_{2}>0$;

(3) $\sum_{n=1}^{m-1}\left\|a_{\tau}^{n}-a_{\tau}^{n-1}\right\|_{L^{\infty}} \leq \kappa$.

Then the solution $\mathbf{v}_{\tau}^{n}$ of the equations

$$
a_{\tau}^{n-1} D_{\tau} \mathbf{v}_{\tau}^{n}-\mathbf{P}_{\mathrm{div}} \Delta \mathbf{v}_{\tau}^{n}=\boldsymbol{f}^{n}, \quad n=1, \ldots, m,
$$

satisfies

$$
\begin{aligned}
& \left\|\left(D_{\tau} \mathbf{v}_{\tau}^{n}\right)_{n=1}^{m}\right\|_{L^{p}\left(L^{q}\right)}+\left\|\left(\mathbf{P}_{\mathrm{div}} \Delta \mathbf{v}_{\tau}^{n}\right)_{n=1}^{m}\right\|_{L^{p}\left(L^{q}\right)} \\
& \quad \leq c\left(\left\|\left(\boldsymbol{f}^{n}\right)_{n=1}^{m}\right\|_{L^{p}\left(L^{q}\right)}+\tau^{\frac{1}{p}-1}\left\|\mathbf{v}_{\tau}^{0}\right\|_{L^{q}}+\tau^{\frac{1}{p}}\left\|\mathbf{P}_{\operatorname{div}} \Delta \mathbf{v}_{\tau}^{0}\right\|_{L^{q}}\right), \quad \forall 1<p, q<\infty,
\end{aligned}
$$

where the constant $c$ is independent of $\tau, m$ and $a_{\tau}^{n-1}, n=1, \ldots, m$ (but may depend on $\kappa_{0}, \kappa_{1}, \kappa_{2}, \sigma, \kappa, p, q$ and $T$ ).

Proof. For $k=1, \ldots, m$, the equation (4.10) can be rewritten as

$$
a_{\tau}^{k-1} D_{\tau} \mathbf{v}_{\tau}^{n}-\mathbf{P}_{\operatorname{div}} \Delta \mathbf{v}_{\tau}^{n}=\boldsymbol{f}^{n}+\left(a_{\tau}^{k-1}-a_{\tau}^{n-1}\right) D_{\tau} \mathbf{v}_{\tau}^{n}
$$

Applying Lemma 4.1 to (4.12) yields

$$
\begin{aligned}
& \left\|\left(D_{\tau} \mathbf{v}_{\tau}^{n}\right)_{n=1}^{k}\right\|_{L^{p}\left(L^{q}\right)}+\left\|\left(\mathbf{P}_{\operatorname{div}} \Delta \mathbf{v}_{\tau}^{n}\right)_{n=1}^{k}\right\|_{L^{p}\left(L^{q}\right)} \\
& \leq c\left(\left\|\left(\boldsymbol{f}^{n}\right)_{n=1}^{k}\right\|_{L^{p}\left(L^{q}\right)}+\tau^{\frac{1}{p}-1}\left\|\mathbf{v}_{\tau}^{0}\right\|_{L^{q}}+\tau^{\frac{1}{p}}\left\|\mathbf{P}_{\operatorname{div}} \Delta \mathbf{v}_{\tau}^{0}\right\|_{L^{q}}\right) \\
& \quad+c\left\|\left(\left(a_{\tau}^{k-1}-a_{\tau}^{n-1}\right) D_{\tau} \mathbf{v}_{\tau}^{n}\right)_{n=1}^{k}\right\|_{L^{p}\left(L^{q}\right)} .
\end{aligned}
$$

Let $E_{0}:=0$ and we define

$$
\begin{aligned}
E_{k} & :=\left\|\left(D_{\tau} \mathbf{v}_{\tau}^{n}\right)_{n=1}^{k}\right\|_{L^{p}\left(L^{q}\right)}^{p}+\left\|\left(\mathbf{P}_{\mathrm{div}} \Delta \mathbf{v}_{\tau}^{n}\right)_{n=1}^{k}\right\|_{L^{p}\left(L^{q}\right)}^{p} \\
& =\tau \sum_{n=1}^{k}\left\|D_{\tau} \mathbf{v}_{\tau}^{n}\right\|_{L^{q}}^{p}+\tau \sum_{n=1}^{k}\left\|\mathbf{P}_{\mathrm{div}} \Delta \mathbf{v}_{\tau}^{n}\right\|_{L^{q}}^{p}, \quad \text { for } \quad k=1, \ldots, m .
\end{aligned}
$$


Then we have

$$
\begin{aligned}
\left\|\left(\left(a_{\tau}^{k-1}-a_{\tau}^{n-1}\right) D_{\tau} \mathbf{v}_{\tau}^{n}\right)_{n=1}^{k}\right\|_{L^{p}\left(L^{q}\right)}^{p} & =\tau \sum_{n=1}^{k}\left\|\left(a_{\tau}^{k-1}-a_{\tau}^{n-1}\right) D_{\tau} \mathbf{v}_{\tau}^{n}\right\|_{L^{q}}^{p} \\
& \leq \tau \sum_{n=1}^{k}\left\|a_{\tau}^{k-1}-a_{\tau}^{n-1}\right\|_{L^{\infty}}^{p}\left\|D_{\tau} \mathbf{v}_{\tau}^{n}\right\|_{L^{q}}^{p} \\
& \leq \tau \sum_{n=1}^{k}\left\|a_{\tau}^{k-1}-a_{\tau}^{n-1}\right\|_{L^{\infty}}^{p}\left(\left\|D_{\tau} \mathbf{v}_{\tau}^{n}\right\|_{L^{q}}^{p}+\left\|\mathbf{P}_{\mathrm{div}} \Delta \mathbf{v}_{\tau}^{n}\right\|_{L^{q}}^{p}\right) \\
& =\sum_{n=1}^{k}\left\|a_{\tau}^{k-1}-a_{\tau}^{n-1}\right\|_{L^{\infty}}^{p}\left(E_{n}-E_{n-1}\right) \\
& =\sum_{n=1}^{k-1}\left(\left\|a_{\tau}^{k-1}-a_{\tau}^{n-1}\right\|_{L^{\infty}}^{p}-\left\|a_{\tau}^{k-1}-a_{\tau}^{n}\right\|_{L^{\infty}}^{p}\right) E_{n} \\
& \leq c \sum_{n=1}^{k-1}\left\|a_{\tau}^{n-1}-a_{\tau}^{n}\right\|_{L^{\infty}} E_{n} .
\end{aligned}
$$

By denoting $F_{k}:=\left\|\left(\boldsymbol{f}_{n}\right)_{n=1}^{k}\right\|_{L^{p}\left(L^{q}\right)}^{p}+\left(\tau^{\frac{1}{p}-1}\left\|\mathbf{v}_{\tau}^{0}\right\|_{L^{q}}+\tau^{\frac{1}{p}}\left\|\mathbf{P}_{\text {div }} \Delta \mathbf{v}_{\tau}^{0}\right\|_{L^{q}}\right)^{p}$ and substituting the last inequality into the $p$ th power of (4.13), we obtain

$$
E_{k} \leq c \sum_{n=1}^{k-1}\left\|a_{\tau}^{n-1}-a_{\tau}^{n}\right\|_{L^{\infty}} E_{n}+c F_{k}, \quad k=1, \ldots, m .
$$

Then applying Gronwall's inequality ( $c f$. [28], Lem. 5.1) yields

$$
E_{m} \leq c F_{m}
$$

The proof of Lemma 4.2 is complete.

\subsection{Proof of Proposition 3.1}

First, we assume that $\rho_{\tau}^{n} \in H^{2}(\Omega) \cap L^{\infty}(\Omega)$ and $\mathbf{u}_{\tau}^{n} \in \mathbf{W}^{1, \infty}(\Omega) \cap \mathbf{H}^{2}(\Omega) \cap \mathbf{H}_{0}^{1}(\Omega)$ are given for $n=$ $0, \ldots, m-1$, such that $\nabla \cdot \mathbf{u}_{\tau}^{n}=0$ and the following inequalities hold for $0 \leq n \leq m-1$ (induction assumption):

$$
\begin{aligned}
\left\|\mathbf{u}_{\tau}^{n}\right\|_{W^{1, \infty}}+\left\|\rho_{\tau}^{n}\right\|_{W^{1, \infty}} & \leq\|\mathbf{u}\|_{C\left([0, T] ; W^{1, \infty}\right)}+\|\rho\|_{C\left([0, T] ; W^{1, \infty}\right)}+1 \\
\min _{x \in \bar{\Omega}} \rho_{\tau}^{0}(x) & \leq \rho_{\tau}^{n}(x) \leq \max _{x \in \bar{\Omega}} \rho_{\tau}^{0}(x), \quad \forall x \in \Omega .
\end{aligned}
$$

Under this assumption, for $n=m$ the hyperbolic equation (3.1) and the parabolic equations (3.2) and (3.3) have a unique solution (the proof is given in Appendix A)

$$
\begin{aligned}
\rho_{\tau}^{m} & \in H^{2}(\Omega) \cap L^{\infty}(\Omega), \\
\left(\mathbf{u}_{\tau}^{m}, p_{\tau}^{m}\right) & \in\left(\mathbf{H}^{2}(\Omega) \cap \mathbf{H}_{0}^{1}(\Omega)\right) \times\left(H^{1}(\Omega) \cap L_{0}^{2}(\Omega)\right),
\end{aligned}
$$

respectively, obeying the following maximum principle:

$$
\min _{x \in \bar{\Omega}} \rho_{\tau}^{m-1}(x) \leq \rho_{\tau}^{m}(x) \leq \max _{x \in \bar{\Omega}} \rho_{\tau}^{m-1}(x), \quad \forall x \in \Omega .
$$


which shows that (4.19) also holds for $n=m$.

Next, we prove (4.18) for $n=m$ to complete the mathematical induction. In the mean time, we also obtain the estimates (3.6)-(3.10) in Proposition 3.1. To this end, we keep the generic constant $c$ of this section to be independent of $m$ (but may depend on $T$ ). The proof consists of two parts: $L^{2}$-type estimates and $W^{1, \infty}$ estimates.

\section{Part I: $L^{2}, H^{1}$ and $H^{2}$ estimates}

We compare the time-discrete solution $\left(\rho_{\tau}^{n}, \mathbf{u}_{\tau}^{n}, p_{\tau}^{n}\right)$ with the PDE's solution $\left(\rho^{n}, \mathbf{u}^{n}, p^{n}\right)$. The latter satisfies the following equations:

$$
\begin{aligned}
D_{\tau} \rho^{n}+\mathbf{u}^{n-1} \cdot \nabla \rho^{n} & =E^{n}, \\
\rho^{n-1} D_{\tau} \mathbf{u}^{n}+\rho^{n} \mathbf{u}^{n-1} \cdot \nabla \mathbf{u}^{n}+\nabla p^{n}-\mu \Delta \mathbf{u}^{n} & =\mathbf{F}^{n}, \\
\nabla \cdot \mathbf{u}^{n} & =0,
\end{aligned}
$$

where

$$
E^{n}=D_{\tau} \rho^{n}-\partial_{t} \rho^{n}+\left(\mathbf{u}^{n-1}-\mathbf{u}^{n}\right) \cdot \nabla \rho^{n}
$$

and

$$
\mathbf{F}^{n}=\rho^{n-1} D_{\tau} \mathbf{u}^{n}-\rho^{n} \partial_{t} \mathbf{u}^{n}+\rho^{n}\left(\mathbf{u}^{n-1}-\mathbf{u}^{n}\right) \cdot \nabla \mathbf{u}^{n}
$$

are truncation errors of the time discretization. Under the regularity assumption (2.15), we have

$$
\left\|E^{n}\right\|_{L^{\infty}}+\left\|E^{n}\right\|_{H^{2}}+\left\|\mathbf{F}^{n}\right\|_{L^{\infty}} \leq c \tau .
$$

Using the notations of (3.5), subtracting (3.1)-(3.3) from (4.23)-(4.25) yields

$$
\begin{aligned}
D_{\tau} e_{\rho}^{n}+\mathbf{u}_{\tau}^{n-1} \cdot \nabla e_{\rho}^{n}+\mathbf{e}_{\mathbf{u}}^{n-1} \cdot \nabla \rho^{n} & =E^{n}, \\
\rho_{\tau}^{n-1} D_{\tau} \mathbf{e}_{\mathbf{u}}^{n}+e_{\rho}^{n-1} D_{\tau} \mathbf{u}^{n}+e_{\rho}^{n} \mathbf{u}^{n-1} \cdot \nabla \mathbf{u}^{n}+\rho_{\tau}^{n} \mathbf{e}_{\mathbf{u}}^{n-1} \cdot \nabla \mathbf{u}^{n}+\rho_{\tau}^{n} \mathbf{u}_{\tau}^{n-1} \cdot \nabla \mathbf{e}_{\mathbf{u}}^{n}+\nabla e_{p}^{n}-\mu \Delta \mathbf{e}_{\mathbf{u}}^{n} & =\mathbf{F}^{n}, \\
\nabla \cdot \mathbf{e}_{\mathbf{u}}^{n} & =0 .
\end{aligned}
$$

For a positive number $k \geq 1$, integrating (4.29) against $\left|e_{\rho}^{n}\right|^{k-1} e_{\rho}^{n}$ yields

$$
\begin{aligned}
& \frac{\left\|e_{\rho}^{n}\right\|_{L^{k+1}}^{k+1}-\left(e_{\rho}^{n-1},\left|e_{\rho}^{n}\right|^{k-1} e_{\rho}^{n}\right)}{\tau}+\int_{\Omega} \nabla \cdot\left(\mathbf{u}_{\tau}^{n-1} \frac{1}{k+1}\left|e_{\rho}^{n}\right|^{k+1}\right) \mathrm{d} x \\
& \quad=\left(E^{n}-\mathbf{e}_{\mathbf{u}}^{n-1} \cdot \nabla \rho^{n},\left|e_{\rho}^{n}\right|^{k-1} e_{\rho}^{n}\right) \\
& \quad \leq\left\|E^{n}\right\|_{L^{k+1}}\left\|e_{\rho}^{n}\right\|_{L^{k+1}}^{k}+\left\|\mathbf{e}_{\mathbf{u}}^{n-1}\right\|_{L^{k+1}}\left\|\nabla \rho^{n}\right\|_{L^{\infty}}\left\|e_{\rho}^{n}\right\|_{L^{k+1}}^{k},
\end{aligned}
$$

where the last step is due to Hölder's inequality: $|(f, g)| \leq\|f\|_{L^{k+1}}\|g\|_{L^{\frac{k+1}{k}}}$. Since

$$
\begin{aligned}
& \int_{\Omega} \nabla \cdot\left(\mathbf{u}_{\tau}^{n-1} \frac{1}{k+1}\left|e_{\rho}^{n}\right|^{k+1}\right) \mathrm{d} x=0, \\
& \frac{\left\|e_{\rho}^{n}\right\|_{L^{k+1}}^{k+1}-\left(e_{\rho}^{n-1},\left|e_{\rho}^{n}\right|^{k-1} e_{\rho}^{n}\right)}{\tau} \geq \frac{\left\|e_{\rho}^{n}\right\|_{L^{k+1}}^{k+1}-\left\|e_{\rho}^{n-1}\right\|_{L^{k+1}}\left\|e_{\rho}^{n}\right\|_{L^{k+1}}^{k}}{\tau},
\end{aligned}
$$

(Hölder's inequality again)

it follows that (4.32) reduces to

$$
\frac{\left\|e_{\rho}^{n}\right\|_{L^{k+1}}-\left\|e_{\rho}^{n-1}\right\|_{L^{k+1}}}{\tau} \leq c\left(\left\|E^{n}\right\|_{L^{k+1}}+\left\|\mathbf{e}_{\mathbf{u}}^{n-1}\right\|_{L^{k+1}}\right) .
$$


By summing up the last inequality for $n=0,1,2, \ldots, m$, we obtain

$$
\max _{0 \leq n \leq m}\left\|e_{\rho}^{n}\right\|_{L^{k+1}} \leq c \sum_{n=1}^{m} \tau\left(\left\|E^{n}\right\|_{L^{k+1}}+\left\|\mathbf{e}_{\mathbf{u}}^{n-1}\right\|_{L^{k+1}}\right) .
$$

Taking $k \rightarrow \infty$ in the last inequality yields

$$
\begin{aligned}
\max _{0 \leq n \leq m}\left\|e_{\rho}^{n}\right\|_{L^{\infty}} & \leq c \sum_{n=1}^{m} \tau\left(\left\|E^{n}\right\|_{L^{\infty}}+\left\|\mathbf{e}_{\mathbf{u}}^{n-1}\right\|_{L^{\infty}}\right) \\
& \leq c \tau+c \sum_{n=1}^{m} \tau\left\|\mathbf{e}_{\mathbf{u}}^{n-1}\right\|_{L^{\infty}},
\end{aligned}
$$

where we have used (4.28) in the last inequality.

The maximum principle (4.22) implies

$$
\min _{x \in \bar{\Omega}} \rho^{0}(x) \leq \rho_{\tau}^{n} \leq \max _{x \in \bar{\Omega}} \rho^{0}(x) .
$$

Since $\left(\nabla e_{p}^{n}, D_{\tau} \mathbf{e}_{\mathbf{u}}^{n}\right)=-\left(e_{p}^{n}, D_{\tau} \nabla \cdot \mathbf{e}_{\mathbf{u}}^{n}\right)=0$, multiplying (4.30) by $D_{\tau} \mathbf{e}_{\mathbf{u}}^{n}$ and integrating the result over $\Omega$ yield

$$
\begin{aligned}
D_{\tau}\left(\frac{\mu}{2}\right. & \left.\left\|\nabla \mathbf{e}_{\mathbf{u}}^{n}\right\|_{L^{2}}^{2}\right)+\left\|\sqrt{\rho_{\tau}^{n-1}} D_{\tau} \mathbf{e}_{\mathbf{u}}^{n}\right\|_{L^{2}}^{2} \\
\leq & \left|\left(e_{\rho}^{n-1} D_{\tau} \mathbf{u}^{n}, D_{\tau} \mathbf{e}_{\mathbf{u}}^{n}\right)\right|+\left|\left(e_{\rho}^{n} \mathbf{u}^{n-1} \cdot \nabla \mathbf{u}^{n}, D_{\tau} \mathbf{e}_{\mathbf{u}}^{n}\right)\right|+\left|\left(\rho_{\tau}^{n} \mathbf{e}_{\mathbf{u}}^{n-1} \cdot \nabla \mathbf{u}^{n}, D_{\tau} \mathbf{e}_{\mathbf{u}}^{n}\right)\right| \\
& +\left|\left(\rho_{\tau}^{n} \mathbf{u}_{\tau}^{n-1} \cdot \nabla \mathbf{e}_{\mathbf{u}}^{n}, D_{\tau} \mathbf{e}_{\mathbf{u}}^{n}\right)\right|+\left|\left(\mathbf{F}^{n}, D_{\tau} \mathbf{e}_{\mathbf{u}}^{n}\right)\right| \\
\leq & \epsilon\left\|D_{\tau} \mathbf{e}_{\mathbf{u}}^{n}\right\|_{L^{2}}^{2}+c \epsilon^{-1}\left(\left\|e_{\rho}^{n-1}\right\|_{L^{2}}^{2}\left\|D_{\tau} \mathbf{u}^{n}\right\|_{L^{\infty}}^{2}+\left\|e_{\rho}^{n}\right\|_{L^{2}}^{2}\left\|\mathbf{u}^{n-1}\right\|_{L^{\infty}}^{2}\left\|\nabla \mathbf{u}^{n}\right\|_{L^{\infty}}^{2}\right) \\
& +c \epsilon^{-1}\left(\left\|\rho_{\tau}^{n}\right\|_{L^{\infty}}^{2}\left\|\mathbf{e}_{\mathbf{u}}^{n-1}\right\|_{L^{2}}^{2}\left\|\nabla \mathbf{u}^{n}\right\|_{L^{\infty}}^{2}+\left\|\rho_{\tau}^{n}\right\|_{L^{\infty}}^{2}\left\|\mathbf{u}_{\tau}^{n-1}\right\|_{L^{\infty}}^{2}\left\|\nabla \mathbf{e}_{\mathbf{u}}^{n}\right\|_{L^{2}}^{2}\right)+c \epsilon^{-1}\left\|\mathbf{F}^{n}\right\|_{L^{2}}^{2} .
\end{aligned}
$$

By choosing a sufficiently small constant $\epsilon$ and using (4.18) and (4.36) to estimate $\left\|\rho_{\tau}^{n}\right\|_{L^{\infty}}$ and $\left\|\mathbf{u}_{\tau}^{n-1}\right\|_{L^{\infty}}$, we obtain

$$
\begin{aligned}
& \frac{1}{2} D_{\tau}\left(\frac{\mu}{2}\left\|\nabla \mathbf{e}_{\mathbf{u}}^{n}\right\|_{L^{2}}^{2}\right)+c^{-1}\left\|D_{\tau} \mathbf{e}_{\mathbf{u}}^{n}\right\|_{L^{2}}^{2} \\
& \quad \leq c\left(\left\|e_{\rho}^{n-1}\right\|_{L^{2}}^{2}+\left\|e_{\rho}^{n}\right\|_{L^{2}}^{2}+\left\|\mathbf{e}_{\mathbf{u}}^{n-1}\right\|_{L^{2}}^{2}+\left\|\nabla \mathbf{e}_{\mathbf{u}}^{n}\right\|_{L^{2}}^{2}+\left\|\mathbf{F}^{n}\right\|_{L^{2}}^{2}\right), \quad n=1, \ldots, m .
\end{aligned}
$$

Then, substituting (4.34) (with $k=1$ ) into the inequality above and summing up the resulting inequalities for $n=1, \ldots, \ell,($ with $\ell \leq m)$ we have

$$
\begin{aligned}
\max _{1 \leq n \leq \ell} & \left\|\nabla \mathbf{e}_{\mathbf{u}}^{n}\right\|_{L^{2}}^{2}+\sum_{n=1}^{\ell} \tau\left\|D_{\tau} \mathbf{e}_{\mathbf{u}}^{n}\right\|_{L^{2}}^{2} \\
\leq & c \sum_{n=1}^{\ell} \tau\left(\left\|\mathbf{e}_{\mathbf{u}}^{n-1}\right\|_{L^{2}}^{2}+\left\|\nabla \mathbf{e}_{\mathbf{u}}^{n}\right\|_{L^{2}}^{2}+\left\|E^{n}\right\|_{L^{2}}^{2}+\left\|\mathbf{F}^{n}\right\|_{L^{2}}^{2}\right) \\
\leq & c \sum_{n=1}^{\ell} \tau\left(\left\|\nabla \mathbf{e}_{\mathbf{u}}^{n}\right\|_{L^{2}}^{2}+\left\|E^{n}\right\|_{L^{2}}^{2}+\left\|\mathbf{F}^{n}\right\|_{L^{2}}^{2}\right), \quad \ell=1, \ldots, m,
\end{aligned}
$$

where we have used the inequality $\left\|\mathbf{e}_{\mathbf{u}}^{n-1}\right\|_{L^{2}} \leq c\left\|\nabla \mathbf{e}_{\mathbf{u}}^{n-1}\right\|_{L^{2}}$ to derive the last inequality. When the step size $\tau$ is smaller than some constant, the last inequality reduces to (through applying Gronwall's inequality)

$$
\max _{1 \leq n \leq m}\left\|\nabla \mathbf{e}_{\mathbf{u}}^{n}\right\|_{L^{2}}^{2}+\sum_{n=1}^{m} \tau\left\|D_{\tau} \mathbf{e}_{\mathbf{u}}^{n}\right\|_{L^{2}}^{2} \leq c \sum_{n=1}^{m} \tau\left(\left\|E^{n}\right\|_{L^{2}}^{2}+\left\|\mathbf{F}^{n}\right\|_{L^{2}}^{2}\right) \leq c \tau^{2},
$$


where we have used (4.28) in the last inequality. Substituting the last inequality into (4.34) (with $k=1$ ) yields

$$
\max _{0 \leq n \leq m}\left\|e_{\rho}^{n}\right\|_{L^{2}} \leq c \tau
$$

In order to derive an $H^{2}$ estimate of $\mathbf{e}_{\mathbf{u}}^{n}$, we consider (4.30) and (4.31), which can be rewritten as

$$
\begin{aligned}
-\mu \Delta \mathbf{e}_{\mathbf{u}}^{n}+\nabla e_{p}^{n}= & \left(\mathbf{F}^{n}-\rho_{\tau}^{n} \mathbf{e}_{\mathbf{u}}^{n-1} \cdot \nabla \mathbf{u}^{n}-\rho_{\tau}^{n} \mathbf{u}_{\tau}^{n-1} \cdot \nabla \mathbf{e}_{\mathbf{u}}^{n}\right) \\
& +\left(-e_{\rho}^{n} \mathbf{u}^{n-1} \cdot \nabla \mathbf{u}^{n}-e_{\rho}^{n-1} D_{\tau} \mathbf{u}^{n}-\rho_{\tau}^{n-1} D_{\tau} \mathbf{e}_{\mathbf{u}}^{n}\right), \\
\nabla \cdot \mathbf{e}_{\mathbf{u}}^{n}= & 0 .
\end{aligned}
$$

The standard $H^{2}$ estimate of Stokes equations (cf. [29]) implies

$$
\begin{aligned}
\sum_{n=1}^{m} \tau\left\|\Delta \mathbf{e}_{\mathbf{u}}^{n}\right\|_{L^{2}}^{2} \leq & \sum_{n=1}^{m} \tau \| \mathbf{F}^{n}-\rho_{\tau}^{n} \mathbf{e}_{\mathbf{u}}^{n-1} \cdot \nabla \mathbf{u}^{n}-\rho_{\tau}^{n} \mathbf{u}_{\tau}^{n-1} \cdot \nabla \mathbf{e}_{\mathbf{u}}^{n} \\
& -e_{\rho}^{n} \mathbf{u}^{n-1} \cdot \nabla \mathbf{u}^{n}-e_{\rho}^{n-1} D_{\tau} \mathbf{u}^{n}-\rho_{\tau}^{n-1} D_{\tau} \mathbf{e}_{\mathbf{u}}^{n} \|_{L^{2}}^{2} \\
\leq & \sum_{n=1}^{m} \tau\left(\left\|\mathbf{F}^{n}\right\|_{L^{2}}+\left\|\rho_{\tau}^{n}\right\|_{L^{\infty}}\left\|\mathbf{e}_{\mathbf{u}}^{n-1}\right\|_{L^{2}}\left\|\nabla \mathbf{u}^{n}\right\|_{L^{\infty}}\right. \\
& +\left\|\rho_{\tau}^{n}\right\|_{L^{\infty}}\left\|\mathbf{u}_{\tau}^{n-1}\right\|_{L^{\infty}}\left\|\nabla \mathbf{e}_{\mathbf{u}}^{n}\right\|_{L^{2}}+\left\|e_{\rho}^{n}\right\|_{L^{2}}\left\|\mathbf{u}^{n-1}\right\|_{L^{\infty}}\left\|\nabla \mathbf{u}^{n}\right\|_{L^{\infty}} \\
& \left.+\left\|e_{\rho}^{n-1}\right\|_{L^{2}}\left\|D_{\tau} \mathbf{u}^{n}\right\|_{L^{\infty}}+\left\|\rho_{\tau}^{n-1}\right\|_{L^{\infty}}\left\|D_{\tau} \mathbf{e}_{\mathbf{u}}^{n}\right\|_{L^{2}}\right)^{2} \\
\leq & c \sum_{n=1}^{m} \tau\left(\left\|\mathbf{F}^{n}\right\|_{L^{2}}^{2}+\left\|\mathbf{e}_{\mathbf{u}}^{n-1}\right\|_{L^{2}}^{2}+\left\|\nabla \mathbf{e}_{\mathbf{u}}^{n}\right\|_{L^{2}}^{2}+\left\|e_{\rho}^{n}\right\|_{L^{2}}^{2}+\left\|D_{\tau} \mathbf{e}_{\mathbf{u}}^{n}\right\|_{L^{2}}^{2}\right) \\
\leq & c \tau^{2}
\end{aligned}
$$

where we have used the induction assumption (4.18) to control $\left\|\mathbf{u}_{\tau}^{n-1}\right\|_{L^{\infty}}$, (4.36) to control $\left\|\rho_{\tau}^{n}\right\|_{L^{\infty}}$, and (4.40), (4.41) to obtain the last inequality. The inequality above implies the following $H^{2}$-norm estimate via the elliptic regularity (for the function $\mathbf{e}_{\mathbf{u}}^{n}$ satisfying the homogeneous Dirichlet boundary condition $\mathbf{e}_{\mathbf{u}}^{n}=0$ on $\partial \Omega$ ):

$$
\sum_{n=1}^{m} \tau\left\|\mathbf{e}_{\mathbf{u}}^{n}\right\|_{H^{2}}^{2} \leq c \sum_{n=1}^{m} \tau\left\|\Delta \mathbf{e}_{\mathbf{u}}^{n}\right\|_{L^{2}}^{2} \leq c \tau^{2}
$$

which further implies

$$
\sum_{n=1}^{m} \tau\left\|D_{\tau} \mathbf{e}_{\mathbf{u}}^{n}\right\|_{H^{2}}^{2} \leq \sum_{n=1}^{m} \tau \frac{2\left\|\mathbf{e}_{\mathbf{u}}^{n}\right\|_{H^{2}}^{2}+2\left\|\mathbf{e}_{\mathbf{u}}^{n}\right\|_{H^{2}}^{2}}{\tau^{2}} \leq c .
$$

Note that (4.45) also implies $\left\|\mathbf{e}_{\mathbf{u}}^{n}\right\|_{H^{2}} \leq c \tau^{\frac{1}{2}}$ and, as a consequence,

$$
\left\|\mathbf{u}_{\tau}^{n}\right\|_{H^{2}}^{2} \leq\left\|\mathbf{u}^{n}\right\|_{H^{2}}^{2}+\left\|\mathbf{e}_{\mathbf{u}}^{n}\right\|_{H^{2}} \leq\left\|\mathbf{u}^{n}\right\|_{H^{2}}^{2}+c \tau^{\frac{1}{2}} \leq c,
$$

which will be needed in Part II. 


\section{Part II: $L^{\infty}$ and $W^{1, \infty}$ estimates}

Since $H^{2}(\Omega) \hookrightarrow L^{\infty}(\Omega)$, the $H^{2}$ estimate (4.45) implies

$$
\begin{aligned}
\sum_{n=1}^{m} \tau\left\|\mathbf{e}_{\mathbf{u}}^{n}\right\|_{L^{\infty}} & \leq\left(\sum_{n=1}^{m} \tau\right)^{\frac{1}{2}}\left(\sum_{n=1}^{m} \tau\left\|\mathbf{e}_{\mathbf{u}}^{n}\right\|_{L^{\infty}}^{2}\right)^{\frac{1}{2}} \quad \text { (Hölder's inequality) } \\
& \leq c T^{\frac{1}{2}}\left(\sum_{n=1}^{m} \tau\left\|\mathbf{e}_{\mathbf{u}}^{n}\right\|_{H^{2}}^{2}\right)^{\frac{1}{2}} \leq c \tau .
\end{aligned}
$$

Substituting the last inequality into (4.35) yields

$$
\max _{0 \leq n \leq m}\left\|e_{\rho}^{n}\right\|_{L^{\infty}} \leq c \tau \text {. }
$$

In view of Section 4.1, we have

$$
\mathbf{P}_{\text {div }}\left(\rho_{\tau}^{n-1} D_{\tau} \mathbf{e}_{\mathbf{u}}^{n}\right)=\rho_{\tau}^{n-1} D_{\tau} \mathbf{e}_{\mathbf{u}}^{n}-\nabla \phi^{n},
$$

where $\phi \in W^{1, q}(\Omega)$, with $\int_{\Omega} \phi^{n} \mathrm{~d} x=0$, is the solution of the equation

$$
\begin{cases}\Delta \phi^{n}=\nabla \cdot\left(\rho_{\tau}^{n-1} D_{\tau} \mathbf{e}_{\mathbf{u}}^{n}\right) & \text { in } \quad \Omega, \\ \nabla \phi^{n} \cdot \mathbf{n}=\rho_{\tau}^{n-1} D_{\tau} \mathbf{e}_{\mathbf{u}}^{n} \cdot \mathbf{n} & \text { on } \quad \partial \Omega\end{cases}
$$

Since $\nabla \cdot \mathbf{e}_{\mathbf{u}}^{n}=0$ in $\Omega$ and $\mathbf{e}_{\mathbf{u}}^{n}=0$ on $\partial \Omega$, the last equation is equivalent to

$$
\begin{cases}\Delta \phi^{n}=\nabla \rho_{\tau}^{n-1} \cdot D_{\tau} \mathbf{e}_{\mathbf{u}}^{n} & \text { in } \quad \Omega, \\ \nabla \phi^{n} \cdot \mathbf{n}=0 & \text { on } \quad \partial \Omega\end{cases}
$$

whose solution satisfies the following standard $H^{2}$ estimate ( $c f$. [22], Thm. 3.1.3.1):

$$
\left\|\phi^{n}\right\|_{H^{2}} \leq c\left\|\nabla \rho_{\tau}^{n-1} \cdot D_{\tau} \mathbf{e}_{\mathbf{u}}^{n}\right\|_{L^{2}}
$$

By the two-dimensional Sobolev embedding $H^{2}(\Omega) \hookrightarrow W^{1, q}(\Omega), \forall 2<q<\infty$, we have

$$
\left\|\nabla \phi^{n}\right\|_{L^{q}} \leq c\left\|\phi^{n}\right\|_{H^{2}} \leq c\left\|\rho_{\tau}^{n-1}\right\|_{W^{1, \infty}}\left\|D_{\tau} \mathbf{e}_{\mathbf{u}}^{n}\right\|_{L^{2}} .
$$

The last inequality, together with (4.18) and (4.40), implies

$$
\left\|\left(\nabla \phi^{n}\right)_{n=1}^{m}\right\|_{L^{2}\left(L^{q}\right)}^{2}=\sum_{n=1}^{m} \tau\left\|\nabla \phi^{n}\right\|_{L^{q}}^{2} \leq c \sum_{n=1}^{m} \tau\left\|D_{\tau} \mathbf{e}_{\mathbf{u}}^{n}\right\|_{L^{2}}^{2} \leq c \tau^{2}, \quad \forall 2<q<\infty .
$$

By using (4.50), equation (4.42) can be rewritten in the following form:

$$
\begin{aligned}
\rho_{\tau}^{n-1} D_{\tau} \mathbf{e}_{\mathbf{u}}^{n}-\mu \mathbf{P}_{\mathrm{div}} \Delta \mathbf{e}_{\mathbf{u}}^{n}= & \mathbf{P}_{\mathrm{div}}\left(\mathbf{F}^{n}-\rho_{\tau}^{n} \mathbf{e}_{\mathbf{u}}^{n-1} \cdot \nabla \mathbf{u}^{n}-\rho_{\tau}^{n} \mathbf{u}_{\tau}^{n-1} \cdot \nabla \mathbf{e}_{\mathbf{u}}^{n}\right) \\
& +\mathbf{P}_{\operatorname{div}}\left(-e_{\rho}^{n} \mathbf{u}^{n-1} \cdot \nabla \mathbf{u}^{n}-e_{\rho}^{n-1} D_{\tau} \mathbf{u}^{n}\right) .
\end{aligned}
$$


Applying Lemma 4.2 (with $p=2$ ) to the above equation yields

$$
\begin{aligned}
&\left\|\left(D_{\tau} \mathbf{e}_{\mathbf{u}}^{n}\right)_{n=1}^{m}\right\|_{L^{2}\left(L^{q}\right)}+\left\|\left(\mathbf{P}_{\mathrm{div}} \Delta \mathbf{e}_{\mathbf{u}}^{n}\right)_{n=1}^{m}\right\|_{L^{2}\left(L^{q}\right)} \\
& \leq c\left\|\left(\nabla \phi^{n}\right)_{n=1}^{m}\right\|_{L^{2}\left(L^{q}\right)}+c\left\|\left(\mathbf{F}^{n}-\rho_{\tau}^{n} \mathbf{e}_{\mathbf{u}}^{n-1} \cdot \nabla \mathbf{u}^{n}-\rho_{\tau}^{n} \mathbf{u}_{\tau}^{n-1} \cdot \nabla \mathbf{e}_{\mathbf{u}}^{n}\right)_{n=1}^{m}\right\|_{L^{2}\left(L^{q}\right)} \\
&+c\left\|\left(-e_{\rho}^{n} \mathbf{u}^{n-1} \cdot \nabla \mathbf{u}^{n}-e_{\rho}^{n-1} D_{\tau} \mathbf{u}^{n}\right)_{n=1}^{m}\right\|_{L^{2}\left(L^{q}\right)} \\
& \leq c\left(\left\|\left(\nabla \phi^{n}\right)_{n=1}^{m}\right\|_{L^{2}\left(L^{q}\right)}+\left\|\left(\mathbf{F}^{n}\right)_{n=1}^{m}\right\|_{L^{2}\left(L^{q}\right)}\right) \\
&+c\left\|\left(\rho_{\tau}^{n}\right)_{n=1}^{m}\right\|_{L^{\infty}\left(L^{\infty}\right)}\left\|\left(\mathbf{e}_{\mathbf{u}}^{n-1}\right)_{n=1}^{m}\right\|_{L^{2}\left(L^{q}\right)}\left\|\left(\nabla \mathbf{u}^{n}\right)_{n=1}^{m}\right\|_{L^{\infty}\left(L^{\infty}\right)} \\
&+c\left\|\left(\rho_{\tau}^{n}\right)_{n=1}^{m}\right\|_{L^{\infty}\left(L^{\infty}\right)}\left\|\left(\mathbf{u}_{\tau}^{n-1}\right)_{n=1}^{m}\right\|_{L^{\infty}\left(L^{\infty}\right)}\left\|\left(\nabla \mathbf{e}_{\mathbf{u}}^{n}\right)_{n=1}^{m}\right\|_{L^{2}\left(L^{q}\right)} \\
&+c\left\|\left(e_{\rho}^{n}\right)_{n=1}^{m}\right\|_{L^{2}\left(L^{q}\right)}\left\|\left(\mathbf{u}^{n-1}\right)_{n=1}^{m}\right\|_{L^{\infty}\left(L^{\infty}\right)}\left\|\left(\nabla \mathbf{u}^{n}\right)_{n=1}^{m}\right\|_{L^{\infty}\left(L^{\infty}\right)} \\
&+c\left\|\left(e_{\rho}^{n-1}\right)_{n=1}^{m}\right\|_{L^{\infty}\left(L^{\infty}\right)}\left\|\left(D_{\tau} \mathbf{u}^{n}\right)_{n=1}^{m}\right\|_{L^{2}\left(L^{q}\right)} \\
&=: J_{1}+J_{2}+J_{3}+J_{4}+J_{5} .
\end{aligned}
$$

By using the induction assumption (4.18) and (4.49), we have

$$
\begin{aligned}
& J_{1} \leq c \tau, \\
& J_{2} \leq c\left\|\left(\mathbf{e}_{\mathbf{u}}^{n-1}\right)_{n=1}^{m}\right\|_{L^{2}\left(L^{q}\right)} \leq c\left\|\left(\mathbf{e}_{\mathbf{u}}^{n-1}\right)_{n=1}^{m}\right\|_{L^{2}\left(H^{2}\right)} \leq c \tau, \\
& J_{3} \leq c\left\|\left(\nabla \mathbf{e}_{\mathbf{u}}^{n-1}\right)_{n=1}^{m}\right\|_{L^{2}\left(L^{q}\right)} \leq c\left\|\left(\mathbf{e}_{\mathbf{u}}^{n-1}\right)_{n=1}^{m}\right\|_{L^{2}\left(H^{2}\right)} \leq c \tau, \\
& J_{4} \leq c\left\|\left(e_{\rho}^{n}\right)_{n=1}^{m}\right\|_{L^{2}\left(L^{q}\right)} \leq c\left\|\left(e_{\rho}^{n}\right)_{n=1}^{m}\right\|_{L^{\infty}\left(L^{\infty}\right)} \leq c \tau, \\
& J_{5} \leq c \tau .
\end{aligned}
$$

By substituting these estimates into (4.57), we obtain

$$
\left\|\left(D_{\tau} \mathbf{e}_{\mathbf{u}}^{n}\right)_{n=1}^{m}\right\|_{L^{2}\left(L^{q}\right)}+\left\|\left(\mathbf{P}_{\operatorname{div}} \Delta \mathbf{e}_{\mathbf{u}}^{n}\right)_{n=1}^{m}\right\|_{L^{2}\left(L^{q}\right)} \leq c \tau .
$$

In any given smooth and convex domain $\Omega$, there exists $q>2$ such that [22]

$$
\left\|\mathbf{e}_{\mathbf{u}}^{n}\right\|_{W^{2, q}} \leq c\left\|\mathbf{P}_{\mathrm{div}} \Delta \mathbf{e}_{\mathbf{u}}^{n}\right\|_{L^{q}} .
$$

Consequently, (4.59) further yields

$$
\left\|\left(D_{\tau} \mathbf{e}_{\mathbf{u}}^{n}\right)_{n=1}^{m}\right\|_{L^{2}\left(L^{q}\right)}+\left\|\left(\mathbf{e}_{\mathbf{u}}^{n}\right)_{n=1}^{m}\right\|_{L^{2}\left(W^{2, q}\right)} \leq c \tau, \quad \text { for some } q>2 \text { (depending on the domain } \Omega \text { ). }
$$

Then the Sobolev embedding $W^{2, q}(\Omega) \hookrightarrow W^{1, \infty}(\Omega)$ (for $q>2$ ) implies

$$
\left\|\left(\mathbf{e}_{\mathbf{u}}^{n}\right)_{n=1}^{m}\right\|_{L^{2}\left(W^{1, \infty}\right)} \leq c \tau .
$$

The last inequality implies $\sum_{n=1}^{m} \tau\left\|\mathbf{e}_{\mathbf{u}}^{n}\right\|_{W^{1, \infty}}^{2} \leq c \tau^{2}$. Since $\mathbf{u}_{\tau}^{n}=\mathbf{u}^{n}-\mathbf{e}_{\mathbf{u}}^{n}$ with $\mathbf{u}^{n}$ being the exact solution of the PDEs, it follows that (for sufficiently small step size $\tau$ )

$$
\left\|\mathbf{u}_{\tau}^{n}\right\|_{W^{1, \infty}} \leq\left\|\mathbf{u}^{n}\right\|_{W^{1, \infty}}+\left\|\mathbf{e}_{\mathbf{u}}^{n}\right\|_{W^{1, \infty}} \leq\left\|\mathbf{u}^{n}\right\|_{W^{1, \infty}}+c \tau^{\frac{1}{2}} \leq\left\|\mathbf{u}^{n}\right\|_{W^{1, \infty}}+1, \quad n=1, \ldots, m .
$$

If we define $\widetilde{\mathbf{e}}_{\mathbf{u}}$, piecewise linear in time, such that

$$
\widetilde{\mathbf{e}}_{\mathbf{u}}(x, t)=\frac{t_{n}-t}{\tau} \mathbf{e}_{\mathbf{u}}^{n-1}(x)+\frac{t-t_{n-1}}{\tau} \mathbf{e}_{\mathbf{u}}^{n}(x) \text { for } t \in\left(t_{n-1}, t_{n}\right],
$$

then (4.60) implies that

$$
\left\|\partial_{t} \widetilde{\mathbf{e}}_{\mathbf{u}}\right\|_{L^{2}\left(0, t_{m} ; L^{q}\right)}+\left\|\widetilde{\mathbf{e}}_{\mathbf{u}}\right\|_{L^{2}\left(0, t_{m} ; W^{2, q}\right)} \leq c \tau
$$


Since $\widetilde{\mathbf{e}}_{\mathbf{u}}(0)=0$, we can further define

$$
\overline{\mathbf{e}}_{\mathbf{u}}(x, t)= \begin{cases}\overline{\mathbf{e}}_{\mathbf{u}}(x, t) & \text { for } t \in\left[0, t_{m}\right], \\ \overline{\mathbf{e}}_{\mathbf{u}}\left(x, 2 t_{m}-t\right) & \text { for } t \in\left[t_{m}, 2 t_{m}\right], \\ 0 & \text { for } t \in\left[2 t_{m}, \infty\right)\end{cases}
$$

which is a function defined for all $t \in \mathbb{R}_{+}$, satisfying

$$
\left\|\partial_{t} \overline{\mathbf{e}}_{\mathbf{u}}\right\|_{L^{2}\left(\mathbb{R}_{+} ; L^{q}\right)}+\left\|\overline{\mathbf{e}}_{\mathbf{u}}\right\|_{L^{2}\left(\mathbb{R}_{+} ; W^{2, q}\right)} \leq c\left(\left\|\partial_{t} \widetilde{\mathbf{e}}_{\mathbf{u}}\right\|_{L^{2}\left(0, t_{m} ; L^{q}\right)}+\left\|\widetilde{\mathbf{e}}_{\mathbf{u}}\right\|_{L^{2}\left(0, t_{m} ; W^{2, q}\right)}\right) \leq c \tau .
$$

Then the inhomogeneous Sobolev embedding ([43], Prop. 1.2.10) implies

$$
\left\|\overline{\mathbf{e}}_{\mathbf{u}}\right\|_{C\left(\left[0, t_{m}\right] ;\left(L^{q}(\Omega), W^{2, q}(\Omega)\right)_{1 / 2,1 / 2}\right)} \leq c\left(\left\|\partial_{t} \overline{\mathbf{e}}_{\mathbf{u}}\right\|_{L^{2}\left(\mathbb{R}_{+} ; L^{q}\right)}+\left\|\overline{\mathbf{e}}_{\mathbf{u}}\right\|_{L^{2}\left(\mathbb{R}_{+} ; W^{2, q}\right)}\right) \leq c \tau,
$$

where the real interpolation space $\left(L^{q}(\Omega), W^{2, q}(\Omega)\right)_{1 / 2,1 / 2}$ coincides with the Besov space $B^{1, q ; 2}(\Omega)$, which is embedded into $L^{\infty}(\Omega)$ for $q>2$ ([2], $\S 7.32$ and $\left.\S 7.34\right)$. Consequently, (4.65) implies

$$
\left\|\widetilde{\mathbf{e}}_{\mathbf{u}}\right\|_{C\left(\left[0, t_{m}\right] ; L^{\infty}\right)} \leq c \tau,
$$

which is equivalent to

$$
\max _{0 \leq n \leq m}\left\|\mathbf{e}_{\mathbf{u}}^{n}\right\|_{L^{\infty}} \leq c \tau
$$

In the following, we estimate $\left\|e_{\rho}^{n}\right\|_{W^{1, \infty}}$ and $\left\|e_{\rho}^{n}\right\|_{H^{2}}$.

Let $e_{\rho, x_{j}}^{n}:=\partial_{x_{j}} e_{\rho}^{n}$ and $\mathbf{e}_{\mathbf{u}, x_{j}}^{n-1}:=\partial_{x_{j}} \mathbf{e}_{\mathbf{u}}^{n-1}$. By differentiating (4.29) with respect to $x_{j}$, we have

$$
D_{\tau} e_{\rho, x_{j}}^{n}+\mathbf{u}_{\tau}^{n-1} \cdot \nabla e_{\rho, x_{j}}^{n}+\mathbf{u}_{\tau, x_{j}}^{n-1} \cdot \nabla e_{\rho}^{n}+\mathbf{e}_{\mathbf{u}, x_{j}}^{n-1} \cdot \nabla \rho^{n}+\mathbf{e}_{\mathbf{u}}^{n-1} \cdot \nabla \rho_{x_{j}}^{n}=E_{x_{j}}^{n} .
$$

Similar as (4.32)-(4.35), integrating (4.68) against $\left|e_{\rho, x_{j}}^{n}\right|^{k-1} e_{\rho, x_{j}}^{n}$ and taking $k \rightarrow \infty$, we have

$$
\begin{aligned}
\max _{1 \leq n \leq m} & \left\|e_{\rho, x_{j}}^{n}\right\|_{L^{\infty}} \\
& \leq c \tau \sum_{n=1}^{m}\left\|\mathbf{u}_{\tau, x_{j}}^{n-1} \cdot \nabla e_{\rho}^{n}+\mathbf{e}_{\mathbf{u}, x_{j}}^{n-1} \cdot \nabla \rho^{n}+\mathbf{e}_{\mathbf{u}}^{n-1} \cdot \nabla \rho_{x_{j}}^{n}-E_{x_{j}}^{n}\right\|_{L^{\infty}} \\
& \leq c \tau \sum_{n=1}^{m}\left\|\nabla \mathbf{u}_{\tau}^{n-1}\right\|_{L^{\infty}}\left\|\nabla e_{\rho}^{n}\right\|_{L^{\infty}}+c \tau \sum_{n=1}^{m}\left(\left\|\nabla \mathbf{e}_{\mathbf{u}}^{n-1}\right\|_{L^{\infty}}+\left\|\mathbf{e}_{\mathbf{u}}^{n-1}\right\|_{L^{\infty}}\right)+c \tau \\
& \leq c \tau \sum_{n=1}^{m}\left\|\nabla e_{\rho}^{n}\right\|_{L^{\infty}}+c \tau
\end{aligned}
$$

where we have used (4.61) and (4.67) to estimate $c \tau \sum_{n=1}^{m}\left(\left\|\nabla \mathbf{e}_{\mathbf{u}}^{n-1}\right\|_{L^{\infty}}+\left\|\mathbf{e}_{\mathbf{u}}^{n-1}\right\|_{L^{\infty}}\right)$, and (4.62) to estimate $\left\|\nabla \mathbf{u}_{\tau}^{n-1}\right\|_{L^{\infty}}$. Since the last inequality holds for $j=1,2$, it follows that

$$
\max _{1 \leq n \leq m}\left\|\nabla e_{\rho}^{n}\right\|_{L^{\infty}} \leq c \tau \sum_{n=1}^{m}\left\|\nabla e_{\rho}^{n}\right\|_{L^{\infty}}+c \tau .
$$

By using the discrete Gronwall's inequality, we obtain (for sufficiently small step size $\tau$ )

$$
\max _{1 \leq n \leq m}\left\|\nabla e_{\rho}^{n}\right\|_{L^{\infty}} \leq c \tau .
$$


In view of (4.62) and (4.72), for sufficiently small step size $\tau$ the mathematical induction (4.18) is closed. Consequently, the estimates (4.36), (4.40), (4.41), (4.45)-(4.67), (4.72) hold for $m=N$ with the same constants. These estimates can be summarized as follows:

$$
\begin{aligned}
& \min _{x \in \bar{\Omega}} \rho^{0}(x) \leq \rho_{\tau}^{n} \leq \max _{x \in \bar{\Omega}} \rho^{0}(x), \quad n=1, \ldots, N, \\
& \max _{1 \leq n \leq N}\left(\left\|e_{\rho}^{n}\right\|_{W^{1, \infty}}+\left\|\mathbf{e}_{\mathbf{u}}^{n}\right\|_{H^{1}}+\left\|\mathbf{e}_{\mathbf{u}}^{n}\right\|_{L^{\infty}}\right) \leq c \tau, \\
& \sum_{n=1}^{N} \tau\left(\left\|D_{\tau} \mathbf{e}_{\mathbf{u}}^{n}\right\|_{L^{2}}^{2}+\left\|\mathbf{e}_{\mathbf{u}}^{n}\right\|_{H^{2}}^{2}\right) \leq c \tau^{2}, \\
& \sum_{n=1}^{N} \tau\left\|D_{\tau} \mathbf{e}_{\mathbf{u}}^{n}\right\|_{H^{2}}^{2} \leq c .
\end{aligned}
$$

Dropping off the exact solutions $\rho^{n}$ and $\mathbf{u}^{n}$ from (4.74), (4.75), and using (4.62), we have

$$
\max _{1 \leq n \leq N}\left(\left\|\mathbf{u}_{\tau}^{n}\right\|_{H^{2}}+\left\|\mathbf{u}_{\tau}^{n}\right\|_{W^{1, \infty}}+\left\|\rho_{\tau}^{n}\right\|_{W^{1, \infty}}+\left\|D_{\tau} \mathbf{u}_{\tau}^{n}\right\|_{L^{\infty}}\right) \leq c .
$$

It remains to estimate $\left\|e_{\rho}^{n}\right\|_{H^{2}}$ and $\left\|e_{p}^{n}\right\|_{H^{1}}$. To estimate the former, we consider the second-order partial derivatives of (4.29), i.e.,

$$
\begin{aligned}
D_{\tau} e_{\rho, x_{i} x_{j}}^{n}= & -\mathbf{u}_{\tau, x_{i} x_{j}}^{n-1} \cdot \nabla e_{\rho}^{n}-\mathbf{u}_{\tau, x_{j}}^{n-1} \cdot \nabla e_{\rho, x_{i}}^{n}-\mathbf{u}_{\tau, x_{i}}^{n-1} \cdot \nabla e_{\rho, x_{j}}^{n}-\mathbf{u}_{\tau}^{n-1} \cdot \nabla e_{\rho, x_{i} x_{j}}^{n} \\
& -\mathbf{e}_{\mathbf{u}, x_{i} x_{j}}^{n-1} \cdot \nabla \rho^{n}-\mathbf{e}_{\mathbf{u}, x_{j}}^{n-1} \cdot \nabla \rho_{x_{i}}^{n} \\
& -\mathbf{e}_{\mathbf{u}, x_{i}}^{n-1} \cdot \nabla \rho_{x_{j}}^{n}-e_{\mathbf{u}}^{n-1} \cdot \nabla \rho_{x_{i} x_{j}}^{n}+E_{x_{i} x_{j}}^{n} .
\end{aligned}
$$

Integrating the equation above against $e_{\rho, x_{i} x_{j}}^{n}$ and summing up the results for $n=1, \ldots, m$, we obtain

$$
\begin{aligned}
\max _{1 \leq n \leq m} & \left\|\nabla^{2} e_{\rho}^{n}\right\|_{L^{2}}^{2} \\
\leq & \tau \sum_{i, j=1}^{2} \sum_{n=1}^{m} \| \mathbf{u}_{\tau, x_{i} x_{j}}^{n-1} \cdot \nabla e_{\rho}^{n}+\mathbf{u}_{\tau, x_{j}}^{n-1} \cdot \nabla e_{\rho, x_{i}}^{n}+\mathbf{u}_{\tau, x_{i}}^{n-1} \cdot \nabla e_{\rho, x_{j}}^{n} \\
& +\mathbf{e}_{\mathbf{u}, x_{i} x_{j}}^{n-1} \cdot \nabla \rho^{n}+\mathbf{e}_{\mathbf{u}, x_{j}}^{n-1} \cdot \nabla \rho_{x_{i}}^{n} \\
& +\mathbf{e}_{\mathbf{u}, x_{i}}^{n-1} \cdot \nabla \rho_{x_{j}}^{n}+\mathbf{e}_{\mathbf{u}}^{n-1} \cdot \nabla \rho_{x_{i} x_{j}}^{n}+E_{x_{i} x_{j}}^{n} \|_{L^{2}}^{2} \\
\leq & \tau \sum_{n=1}^{m}\left(\left\|\nabla^{2} \mathbf{u}_{\tau}^{n-1}\right\|_{L^{2}}^{2}\left\|\nabla e_{\rho}^{n}\right\|_{L^{\infty}}^{2}+\left\|\nabla \mathbf{u}_{\tau}^{n-1}\right\|_{L^{\infty}}^{2}\left\|\nabla^{2} e_{\rho}^{n}\right\|_{L^{2}}^{2}\right. \\
& +\left\|\mathbf{e}_{\mathbf{u}}^{n-1}\right\|_{L^{2}}^{2}\left\|\nabla \rho^{n}\right\|_{L^{\infty}}^{2}+\left\|\nabla \mathbf{e}_{\mathbf{u}}^{n-1}\right\|_{L^{\infty}}^{2}\left\|\nabla^{2} \rho^{n}\right\|_{L^{2}}^{2} \\
& \left.+\left\|\nabla \mathbf{e}_{\mathbf{u}}^{n-1}\right\|_{L^{\infty}}^{2}\left\|\nabla^{2} \rho^{n}\right\|_{L^{2}}^{2}+\left\|\mathbf{e}_{\mathbf{u}}^{n-1}\right\|_{L^{\infty}}^{2}\left\|\nabla^{3} \rho^{n}\right\|_{L^{2}}^{2}+\left\|\nabla^{2} E^{n}\right\|_{L^{2}}^{2}\right) \\
= & I_{1}+I_{2}+I_{3}+I_{4}+I_{5}+I_{6}+I_{7},
\end{aligned}
$$

where

$$
\begin{aligned}
& I_{1} \leq c \tau^{2} \\
& \text { (use (4.47) and (4.72)) } \\
& I_{2} \leq c \tau \sum_{n=1}^{m}\left\|\nabla^{2} e_{\rho}^{n}\right\|_{L^{2}}^{2} \\
& \text { (use (4.62)) } \\
& I_{3} \leq c \tau^{2} \\
& \text { (use (4.45)) } \\
& I_{4} \leq c \tau^{2} \\
& I_{5} \leq c \tau^{2} \\
& \text { (use (4.61)) } \\
& I_{6} \leq c \tau^{2} \\
& \text { (the same as } I_{4} \text { ) } \\
& I_{7} \leq c \tau^{2} \text {. } \\
& \text { (use (4.61)) } \\
& \text { (use (4.28)) }
\end{aligned}
$$


Substituting these estimates into (4.79) yields

$$
\max _{1 \leq n \leq m}\left\|\nabla^{2} e_{\rho}^{n}\right\|_{L^{2}}^{2} \leq c \tau \sum_{n=1}^{m}\left\|\nabla^{2} e_{\rho}^{n}\right\|_{L^{2}}^{2}+c \tau^{2},
$$

which holds for all $m=1, \ldots, N$. By using Gronwall's inequality, we derive (for sufficiently small step size $\tau$ )

$$
\max _{1 \leq n \leq N}\left\|\nabla^{2} e_{\rho}^{n}\right\|_{L^{2}} \leq c \tau
$$

Finally, we estimate $\left\|\nabla e_{p}^{n}\right\|_{L^{2}}$. To this end, we rewrite (4.30) as

$$
\begin{aligned}
\nabla e_{p}^{n}= & -\rho_{\tau}^{n-1} D_{\tau} \mathbf{e}_{\mathbf{u}}^{n}-e_{\rho}^{n-1} D_{\tau} \mathbf{u}^{n}+\mu \Delta \mathbf{e}_{\mathbf{u}}^{n}-e_{\rho}^{n} \mathbf{u}^{n-1} \cdot \nabla \mathbf{u}^{n} \\
& -\rho_{\tau}^{n} \mathbf{e}_{\mathbf{u}}^{n-1} \cdot \nabla \mathbf{u}^{n}-\rho_{\tau}^{n} \mathbf{u}_{\tau}^{n-1} \cdot \nabla \mathbf{e}_{\mathbf{u}}^{n}+\mathbf{F}^{n} .
\end{aligned}
$$

By using (4.18), (4.36) and (4.62), we have

$$
\begin{aligned}
\left\|\nabla e_{p}^{n}\right\|_{L^{2}} \leq & c\left(\left\|\rho_{\tau}^{n-1}\right\|_{L^{\infty}}\left\|D_{\tau} \mathbf{e}_{\mathbf{u}}^{n}\right\|_{L^{2}}+\left\|D_{\tau} \mathbf{u}^{n}\right\|_{L^{\infty}}\left\|e_{\rho}^{n-1}\right\|_{L^{2}}+\left\|\mathbf{e}_{\mathbf{u}}^{n}\right\|_{H^{2}}\right. \\
& +\left\|\mathbf{u}^{n-1}\right\|_{L^{\infty}}\left\|\nabla \mathbf{u}^{n}\right\|_{L^{\infty}}\left\|e_{\rho}^{n}\right\|_{L^{2}}+\left\|\rho_{\tau}^{n}\right\|_{L^{\infty}}\left\|\nabla \mathbf{u}^{n}\right\|_{L^{\infty}}\left\|\mathbf{e}_{\mathbf{u}}^{n-1}\right\|_{L^{2}} \\
& \left.+\left\|\rho_{\tau}^{n}\right\|_{L^{\infty}}\left\|\mathbf{u}_{\tau}^{n-1}\right\|_{L^{\infty}}\left\|\nabla \mathbf{e}_{\mathbf{u}}^{n}\right\|_{L^{2}}+\left\|\mathbf{F}^{n}\right\|_{L^{2}}\right) \\
\leq & c\left(\left\|D_{\tau} \mathbf{e}_{\mathbf{u}}^{n}\right\|_{L^{2}}+\left\|e_{\rho}^{n-1}\right\|_{L^{2}}+\left\|\mathbf{e}_{\mathbf{u}}^{n}\right\|_{H^{2}}+\left\|e_{\rho}^{n}\right\|_{L^{2}}+\left\|\mathbf{e}_{\mathbf{u}}^{n-1}\right\|_{L^{2}}+\left\|\nabla \mathbf{e}_{\mathbf{u}}^{n}\right\|_{L^{2}}+\left\|\mathbf{F}^{n}\right\|_{L^{2}}\right) .
\end{aligned}
$$

The last inequality, together with (4.40), (4.41) and (4.45), implies

$$
\tau \sum_{n=1}^{N}\left\|\nabla e_{p}^{n}\right\|_{L^{2}}^{2} \leq c \tau^{2}
$$

\section{Part III: Summary of the proof}

(4.73) implies (3.6).

(4.74) and (4.81) imply (3.7).

(4.75) and (4.83) imply (3.8).

(4.77), (4.81) and (4.83) imply (3.9).

(4.76) and (4.83) imply (3.10).

The proof of Proposition 3.1 is complete.

\section{Proof of Proposition 3.2}

In this section we estimate the spatial discretization errors by comparing the fully discrete finite element solution $\left(\rho_{h}^{n}, \mathbf{u}_{h}^{n}, p_{h}^{n}\right)$ with the semi-discrete solution $\left(\rho_{\tau}^{n}, \mathbf{u}_{\tau}^{n}, p_{\tau}^{n}\right)$. The solvability of the linear system (2.11) and (2.12) is proved in the next subsection.

\subsection{Solvability of the linear system}

If $\left(\rho_{h}^{k}, \mathbf{u}_{h}^{k}, p_{h}^{k}\right) \in M_{h}^{2} \times \mathbf{X}_{h}^{2} \times \stackrel{\circ}{M}_{h}^{1}$ is given for $k=0, \ldots, n-1$, then the discrete linear problem (2.11) has a unique solution $\rho_{h}^{n} \in M_{h}^{2}$ if and only if the corresponding homogeneous problem

$$
\left(\tau^{-1} \Phi_{h}, \varphi_{h}\right)+\left(\mathbf{u}_{h}^{n-1} \cdot \nabla \Phi_{h}, \varphi_{h}\right)+\frac{1}{2}\left(\Phi_{h} \nabla \cdot \mathbf{u}_{h}^{n-1}, \varphi_{h}\right)=0, \quad \forall \varphi_{h} \in M_{h}^{2}
$$


has only zero solution $\Phi_{h}=0$. Indeed, the equation above can be rewritten as, through integration by parts,

$$
\left(\tau^{-1} \Phi_{h}, \varphi_{h}\right)+\frac{1}{2}\left(\mathbf{u}_{h}^{n-1} \cdot \nabla \Phi_{h}, \varphi_{h}\right)-\frac{1}{2}\left(\mathbf{u}_{h}^{n-1} \cdot \nabla \varphi_{h}, \Phi_{h}\right)=0 .
$$

Substituting $\varphi_{h}=\Phi_{h}$ into the equation above immediately yields $\Phi_{h}=0$. This proves the unique solvability of equation (2.11).

After solving $\rho_{h}^{n}$ from (2.11), the truncated functions $\widehat{\rho}_{h}^{n-1}$ and $\widehat{\rho}_{h}^{n}$ can be defined by (2.10), and the discrete linear problem (2.12) has a unique solution $\left(\mathbf{u}_{h}^{n}, p_{h}^{n}\right) \in \mathbf{X}_{h}^{2} \times \stackrel{\circ}{M}_{h}^{1}$ if and only if the corresponding homogeneous problem

$$
\begin{aligned}
\left(\widehat{\rho}_{h}^{n-1} \tau^{-1} \mathbf{U}_{h}, \mathbf{v}_{h}\right) & +\frac{1}{2}\left(\tau^{-1}\left(\widehat{\rho}_{h}^{n}-\widehat{\rho}_{h}^{n-1}\right) \mathbf{U}_{h}, \mathbf{v}_{h}\right)+\frac{1}{2}\left(\nabla \cdot\left(\rho_{h}^{n} \mathbf{u}_{h}^{n-1}\right) \mathbf{U}_{h}, \mathbf{v}_{h}\right) \\
& +\left(\rho_{h}^{n} \mathbf{u}_{h}^{n-1} \cdot \nabla \mathbf{U}_{h}, \mathbf{v}_{h}\right)+B\left(\left(\mathbf{U}_{h}, P_{h}\right),\left(\mathbf{v}_{h}, q_{h}\right)\right)=0, \quad \forall\left(\mathbf{v}_{h}, q_{h}\right) \in \mathbf{X}_{h}^{2} \times \stackrel{\circ}{M}_{h}^{1},
\end{aligned}
$$

has only zero solution $\left(\mathbf{U}_{h}, P_{h}\right)=(\mathbf{0}, 0)$. Indeed, substituting $\left(\mathbf{v}_{h}, q_{h}\right)=\left(\mathbf{U}_{h}, P_{h}\right)$ into the equation above yields

$$
\frac{1}{2 \tau}\left(\left(\widehat{\rho}_{h}^{n-1}+\hat{\rho}_{h}^{n}\right) \mathbf{U}_{h}, \mathbf{v}_{h}\right)+\left(\mu \nabla \mathbf{U}_{h}, \nabla \mathbf{v}_{h}\right)=0 .
$$

Since the truncation operation defined in (2.10) implies $\widehat{\rho}_{h}^{n-1}>0$ and $\widehat{\rho}_{h}^{n}>0$, the last equation implies $\mathbf{U}_{h}=\mathbf{0}$. Then (5.3) reduces to

$$
\left(P_{h}, \nabla \cdot \mathbf{v}_{h}\right)=0, \quad \forall \mathbf{v}_{h} \in \mathbf{X}_{h}^{2},
$$

which implies $\left\|P_{h}\right\|_{L^{2}}=0$ in view of the inf-sup condition (2.9). This proves the unique solvability of equation (2.12).

\subsection{Ritz projection, $L^{2}$ projections, and Lagrange interpolations}

To obtain error estimates for the finite element spatial discretization, we need to use the Stokes-Ritz projection $\left(\mathbf{R}_{h}, Q_{h}\right): \mathbf{H}_{0}^{1}(\Omega) \times L^{2}(\Omega) \rightarrow \mathbf{X}_{h}^{2} \times \stackrel{\circ}{M}_{h}^{1}$, defined by

$$
B\left(\left(\mathbf{R}_{h}(\mathbf{u}, p), Q_{h}(\mathbf{u}, p)\right),\left(\mathbf{v}_{h}, q_{h}\right)\right)=B\left((\mathbf{u}, p),\left(\mathbf{v}_{h}, q_{h}\right)\right), \quad \forall\left(\mathbf{v}_{h}, q_{h}\right) \in \mathbf{X}_{h}^{2} \times \stackrel{\circ}{M}_{h}^{1} .
$$

The $L^{2}$ projections $P_{h}: L^{2}(\Omega) \rightarrow M_{h}^{2}$ and $\mathbf{P}_{h}: \mathbf{L}^{2}(\Omega) \rightarrow \mathbf{X}_{h}^{2}$ defined below will also be used:

$$
\begin{array}{lll}
\left(\mathbf{v}-\mathbf{P}_{h} \mathbf{v}, \mathbf{v}_{h}\right)=0 & \forall \mathbf{v} \in \mathbf{L}^{2}(\Omega), & \forall \mathbf{v}_{h} \in \mathbf{X}_{h}^{2}, \\
\left(v-P_{h} v, v_{h}\right)=0 & \forall v \in L^{2}(\Omega), & \forall v_{h} \in M_{h}^{2} .
\end{array}
$$

It is well known that the Ritz projection and the $L^{2}$ projections defined above satisfy the following standard estimates (cf. [9], Thm. 12.6.7, [20], Chap. II, Thm. 4.3, [21], Thm. 8.2, [13]):

$$
\begin{array}{ll}
\left\|\mathbf{R}_{h}(\mathbf{v}, q)-\mathbf{v}\right\|_{L^{2}} \leq c h^{\ell+1}\left(\|\mathbf{v}\|_{H^{\ell+1}}+\|q\|_{H^{\ell}}\right), & \ell=1,2, \\
\left\|\mathbf{R}_{h}(\mathbf{v}, q)-\mathbf{v}\right\|_{H^{1}}+\left\|Q_{h}(\mathbf{v}, q)-q\right\|_{L^{2}} \leq c h^{\ell}\left(\|\mathbf{v}\|_{H^{\ell+1}}+\|q\|_{H^{\ell}}\right), & \ell=1,2, \\
\left\|\mathbf{R}_{h}(\mathbf{v}, q)\right\|_{W^{1, \infty}} \leq c\left(\|\mathbf{v}\|_{W^{1, \infty}}+\|q\|_{L^{\infty}}\right), & \\
\left\|\mathbf{v}-\mathbf{P}_{h} \mathbf{v}\right\|_{L^{2}} \leq c h^{\ell+1}\|\mathbf{v}\|_{H^{\ell+1}}, & \ell=0,1,2, \\
\left\|\varphi-P_{h} \varphi\right\|_{L^{2}} \leq c h^{\ell+1}\|\varphi\|_{H^{\ell+1}}, & \ell=0,1,2, \\
\left\|P_{h} \varphi\right\|_{W^{k, q}} \leq c\|\varphi\|_{W^{k, q}}, & k=0,1,1 \leq q \leq \infty, \\
\left\|\mathbf{P}_{h} \mathbf{w}\right\|_{L^{2}} \leq c\|\mathbf{w}\|_{L^{2}}, \quad\left\|\mathbf{P}_{h} \mathbf{w}\right\|_{H^{1}} \leq c\|\mathbf{w}\|_{H^{1}}, &
\end{array}
$$


where $(\mathbf{v}, q) \in\left(\mathbf{H}^{\ell+1}(\Omega) \cap \mathbf{H}_{0}^{1}(\Omega)\right) \times H^{\ell}(\Omega), \varphi \in H^{\ell+1}(\Omega)$ and $\mathbf{w} \in \mathbf{H}_{0}^{1}(\Omega)$. Similarly, the Lagrangian interpolation operators satisfy

$$
\begin{array}{lll}
\left\|\Pi_{h} \varphi-\varphi\right\|_{L^{2}}+h\left\|\nabla\left(\Pi_{h} \varphi-\varphi\right)\right\|_{L^{2}} \leq c h^{\ell+1}\|\varphi\|_{H^{\ell+1}}, & \forall \varphi \in H^{\ell+1}(\Omega), & \ell=1,2, \\
\left\|\Pi_{h} \mathbf{v}-\mathbf{v}\right\|_{L^{2}}+h\left\|\nabla\left(\Pi_{h} \mathbf{v}-\mathbf{v}\right)\right\|_{L^{2}} \leq c h^{\ell+1}\|\mathbf{v}\|_{H^{\ell+1}}, & \forall \mathbf{v} \in \mathbf{H}^{\ell+1}(\Omega) \cap \mathbf{H}_{0}^{1}(\Omega), & \ell=1,2, \\
\left\|\Pi_{h} \mathbf{v}-\mathbf{v}\right\|_{L^{\infty}} \leq c h\|\mathbf{v}\|_{W^{1, \infty}}, & \forall \mathbf{v} \in \mathbf{W}^{1, \infty}(\Omega) \cap \mathbf{H}_{0}^{1}(\Omega) . &
\end{array}
$$

The estimates (5.6)-(5.14) will be frequently used in this section.

In view of (3.5), we also have the following estimates of the $L^{2}$ and Ritz projections:

$$
\begin{aligned}
\left\|\rho_{\tau}^{n}-P_{h} \rho_{\tau}^{n}\right\|_{L^{2}} & \leq\left\|e_{\rho}^{n}-P_{h} e_{\rho}^{n}\right\|_{L^{2}}+\left\|\rho^{n}-P_{h} \rho^{n}\right\|_{L^{2}} \\
& \leq c h^{2}\left\|e_{\rho}^{n}\right\|_{H^{2}}+c h^{3}\left\|\rho^{n}\right\|_{H^{3}} \\
& \leq c h^{2} \tau+c h^{3}, \quad\left(\text { use }(3.7) \text { to estimate }\left\|e_{\rho}^{n}\right\|_{H^{2}}\right) \\
\left\|\rho_{\tau}^{n}-P_{h} \rho_{\tau}^{n}\right\|_{H^{1}} & \leq\left\|e_{\rho}^{n}-P_{h} e_{\rho}^{n}\right\|_{H^{1}}+\left\|\rho^{n}-P_{h} \rho^{n}\right\|_{H^{1}} \\
& \leq c h\left\|e_{\rho}^{n}\right\|_{H^{2}}+c h^{2}\left\|\rho^{n}\right\|_{H^{3}} \\
& \leq c h \tau+c h^{2}, \quad\left(\text { use }(3.7) \text { to estimate }\left\|e_{\rho}^{n}\right\|_{H^{2}}\right) \\
\left\|\mathbf{u}_{\tau}^{n}-\mathbf{R}_{h}\left(\mathbf{u}_{\tau}^{n}, p_{\tau}^{n}\right)\right\|_{L^{2}} & \leq\left\|\mathbf{e}_{\mathbf{u}}^{n}-\mathbf{R}_{h}\left(\mathbf{e}_{\mathbf{u}}^{n}, e_{p}^{n}\right)\right\|_{L^{2}}+\left\|\mathbf{u}^{n}-\mathbf{R}_{h}\left(\mathbf{u}^{n}, p^{n}\right)\right\|_{L^{2}} \\
& \leq c h^{2}\left(\left\|\mathbf{e}_{\mathbf{u}}^{n}\right\|_{H^{2}}+\left\|e_{p}^{n}\right\|_{H^{1}}\right)+c h^{3}\left(\left\|\mathbf{u}^{n}\right\|_{H^{3}}+\left\|p^{n}\right\|_{H^{2}}\right) \\
& \leq c h^{2} \tau^{\frac{1}{2}}+c h^{3}, \quad\left(\text { use }(3.8) \text { to estimate }\left\|\mathbf{e}_{\mathbf{u}}^{n}\right\|_{H^{2}}+\left\|e_{p}^{n}\right\|_{H^{1}}\right) \\
\left\|\mathbf{u}_{\tau}^{n}-\mathbf{R}_{h}\left(\mathbf{u}_{\tau}^{n}, p_{\tau}^{n}\right)\right\|_{H^{1}} & \leq\left\|\mathbf{e}_{\mathbf{u}}^{n}-\mathbf{R}_{h}\left(\mathbf{e}_{\mathbf{u}}^{n}, e_{p}^{n}\right)\right\|_{H^{1}}+\left\|\mathbf{u}^{n}-\mathbf{R}_{h}\left(\mathbf{u}^{n}, p^{n}\right)\right\|_{H^{1}} \\
& \leq c h\left(\left\|\mathbf{e}_{\mathbf{u}}^{n}\right\|_{H^{2}}+\left\|e_{p}^{n}\right\|_{H^{1}}\right)+c h^{2}\left(\left\|\mathbf{u}^{n}\right\|_{H^{3}}+\left\|p^{n}\right\|_{H^{2}}\right) \\
& \leq c h \tau^{\frac{1}{2}}+c h^{2} . \quad\left(\text { use }(3.8) \text { to estimate }\left\|\mathbf{e}_{\mathbf{u}}^{n}\right\|_{H^{2}}+\left\|e_{p}^{n}\right\|_{H^{1}}\right)
\end{aligned}
$$

\subsection{Methodology}

Instead of considering the original scheme (2.11) and (2.12) directly, we consider the following finite element equations:

$$
\begin{aligned}
\left(D_{\tau} \rho_{h}^{n}, \varphi_{h}\right)+\left(\mathbf{u}_{h}^{n-1} \cdot \nabla \rho_{h}^{n}, \varphi_{h}\right)+\frac{1}{2}\left(\nabla \cdot \mathbf{u}_{h}^{n-1} \rho_{h}^{n}, \varphi_{h}\right)=0 \\
\left(\rho_{h}^{n-1} D_{\tau} \mathbf{u}_{h}^{n}, \mathbf{v}_{h}\right)+\frac{1}{2}\left(D_{\tau} \rho_{h}^{n} \mathbf{u}_{h}^{n}, \mathbf{v}_{h}\right)+\frac{1}{2}\left(\nabla \cdot\left(\rho_{h}^{n} \mathbf{u}_{h}^{n-1}\right) \mathbf{u}_{h}^{n}, \mathbf{v}_{h}\right) \\
+\left(\rho_{h}^{n} \mathbf{u}_{h}^{n-1} \cdot \nabla \mathbf{u}_{h}^{n}, \mathbf{v}_{h}\right)+B\left(\left(\mathbf{u}_{h}^{n}, p_{h}^{n}\right),\left(\mathbf{v}_{h}, q_{h}\right)\right)=0
\end{aligned}
$$

which do not truncate $\rho_{h}^{n-1}$ and $\rho_{h}^{n}$ in (5.21). We shall prove that, for sufficiently small $\tau$ and $h$, the finite element solutions given by (5.20) and (5.21) satisfy

$$
\frac{1}{2} \min _{y \in \bar{\Omega}} \rho^{0}(y) \leq \rho_{h}^{n} \leq \frac{3}{2} \max _{y \in \bar{\Omega}} \rho^{0}(y), \quad n=0,1, \ldots, N,
$$

which implies $\widehat{\rho}_{h}^{n}=\rho_{h}^{n}$ in view of the definition of the truncation (2.10). In other words, the solutions of (5.20) and (5.21) coincide with the solutions of (2.11) and (2.12). Thus it suffices to present error estimates for the solutions of (5.20) and (5.21).

For the solution $\left(\rho_{h}^{n}, \mathbf{u}_{h}^{n}, p_{h}^{n}\right)$ of (5.20) and (5.21), we denote

$$
e_{\rho, h}^{n}=P_{h} \rho_{\tau}^{n}-\rho_{h}^{n}, \quad \mathbf{e}_{\mathbf{u}, h}^{n}=\mathbf{R}_{h}\left(\mathbf{u}_{\tau}^{n}, p_{\tau}^{n}\right)-u_{h}^{n}, \quad e_{p, h}^{n}=Q_{h}\left(\mathbf{u}_{\tau}^{n}, p_{\tau}^{n}\right)-p_{h}^{n} .
$$


If we can prove (5.22) and

$$
\max _{1 \leq n \leq N}\left(\left\|\mathbf{e}_{\mathbf{u}, h}^{n}\right\|_{L^{2}}+\left\|e_{\rho, h}^{n}\right\|_{L^{2}}\right)+h\left(\tau \sum_{n=1}^{N}\left\|e_{p, h}^{n}\right\|_{L^{2}}^{2}\right)^{\frac{1}{2}} \leq \operatorname{ch} \sqrt{\tau+h^{2}},
$$

then $\left(\rho_{h}^{n}, \mathbf{u}_{h}^{n}, p_{h}^{n}\right), n=1, \ldots, N$, coincide with the solutions of (2.11), (2.12) and

$$
\begin{aligned}
\left\|\rho^{n}-\rho_{h}^{n}\right\|_{L^{2}} & \leq c\left\|\rho^{n}-\rho_{\tau}^{n}\right\|_{L^{2}}+\left\|\rho_{\tau}^{n}-P_{h} \rho_{\tau}^{n}\right\|_{L^{2}}+\left\|e_{\rho, h}^{n}\right\|_{L^{2}} \\
& \leq c \tau+c h^{2}\left\|\rho_{\tau}^{n}\right\|_{H^{2}}+c h \sqrt{\tau+h^{2}} \quad \text { (use (3.7), (3.9), (5.10) and (5.23)) } \\
& \leq c\left(\tau+h^{2}\right), \\
\left\|\mathbf{u}^{n}-\mathbf{u}_{h}^{n}\right\|_{L^{2}} & \leq c\left\|\mathbf{u}^{n}-\mathbf{u}_{\tau}^{n}\right\|_{L^{2}}+\left\|\mathbf{u}_{\tau}^{n}-\mathbf{R}_{h}\left(\mathbf{u}_{\tau}^{n}, p_{\tau}^{n}\right)\right\|_{L^{2}}+\left\|\mathbf{e}_{\mathbf{u}, h}^{n}\right\|_{L^{2}} \\
& \leq c \tau+c h^{2}\left(\left\|\mathbf{u}_{\tau}^{n}\right\|_{H^{2}}+\left\|p_{\tau}^{n}\right\|_{H^{1}}\right)+c h \sqrt{\tau+h^{2}} \quad \text { (use (3.7), (3.9), (5.6) and (5.23)) } \\
& \leq c\left(\tau+h^{2}\right), \\
\left\|p^{n}-p_{h}^{n}\right\|_{L^{2}} & \leq c\left\|p^{n}-p_{\tau}^{n}\right\|_{L^{2}}+\left\|p_{\tau}^{n}-Q_{h}\left(\mathbf{u}_{\tau}^{n}, p_{\tau}^{n}\right)\right\|_{L^{2}}+\left\|e_{p, h}^{n}\right\|_{L^{2}} \\
& \leq c\left\|e_{p}^{n}\right\|_{H^{1}}+c h\left(\left\|\mathbf{u}_{\tau}^{n}\right\|_{H^{2}}+\left\|p_{\tau}^{n}\right\|_{H^{1}}\right)+c\left\|e_{p, h}^{n}\right\|_{L^{2}} \quad \text { (use (3.9) and (5.7)) } \\
& \leq c\left\|e_{p}^{n}\right\|_{H^{1}}+c\left\|e_{p, h}^{n}\right\|_{L^{2}}+c h .
\end{aligned}
$$

The last inequality, (3.8) and (5.23) imply

$$
\left(\tau \sum_{n=1}^{N}\left\|p^{n}-p_{h}^{n}\right\|_{L^{2}}^{2}\right)^{\frac{1}{2}} \leq c \sqrt{\tau+h^{2}} .
$$

This proves the error estimate in Proposition 3.2.

It remains to prove (5.22) and (5.23). To this end, we integrate (3.1)-(3.3) against some test functions and reformulate the equations as

$$
\begin{aligned}
&\left(D_{\tau} \rho_{\tau}^{n}, \varphi_{h}\right)+\left(\mathbf{u}_{\tau}^{n-1} \cdot \nabla \rho_{\tau}^{n}, \varphi_{h}\right)+\frac{1}{2}\left(\nabla \cdot \mathbf{u}_{\tau}^{n-1} \rho_{\tau}^{n}, \varphi_{h}\right)=0 \\
&\left(\rho_{\tau}^{n-1} D_{\tau} \mathbf{u}_{\tau}^{n}, \mathbf{v}_{h}\right)+ \frac{1}{2}\left(D_{\tau} \rho_{\tau}^{n} \mathbf{u}_{\tau}^{n}, \mathbf{v}_{h}\right)+\frac{1}{2}\left(\nabla \cdot\left(\rho_{\tau}^{n} \mathbf{u}_{\tau}^{n-1}\right) \mathbf{u}_{\tau}^{n}, \mathbf{v}_{h}\right) \\
&+\left(\rho_{\tau}^{n} \mathbf{u}_{\tau}^{n-1} \cdot \nabla \mathbf{u}_{\tau}^{n}, \mathbf{v}_{h}\right)+B\left(\left(\mathbf{u}_{\tau}^{n}, p_{\tau}^{n}\right),\left(\mathbf{v}_{h}, q_{h}\right)\right)=0
\end{aligned}
$$

where $\varphi_{h} \in M_{h}^{2}$ and $\left(\mathbf{v}_{h}, q_{h}\right) \in \mathbf{X}_{h}^{2} \times \stackrel{\circ}{M}_{h}^{1}$ are arbitrary test functions. We have added the following stabilization terms to (5.24) and (5.25):

$$
\frac{1}{2} \nabla \cdot \mathbf{u}_{\tau}^{n-1} \rho_{\tau}^{n}=0, \quad \frac{1}{2} D_{\tau} \rho_{\tau}^{n} \mathbf{u}_{\tau}^{n}+\frac{1}{2} \nabla \cdot\left(\rho_{\tau}^{n} \mathbf{u}_{\tau}^{n-1}\right) \mathbf{u}_{\tau}^{n}=0,
$$

which are consequences of (3.3) and (3.1), respectively. Using integration by parts, (5.25) can be further written as

$$
\begin{aligned}
\left(\rho_{\tau}^{n-1} D_{\tau} \mathbf{u}_{\tau}^{n}, \mathbf{v}_{h}\right) & +\frac{1}{2}\left(D_{\tau} \rho_{\tau}^{n} \mathbf{u}_{\tau}^{n}, \mathbf{v}_{h}\right)+\frac{1}{2}\left(\rho_{\tau}^{n} \mathbf{u}_{\tau}^{n-1} \cdot \nabla \mathbf{u}_{\tau}^{n}, \mathbf{v}_{h}\right) \\
& -\frac{1}{2}\left(\rho_{\tau}^{n} \mathbf{u}_{\tau}^{n-1} \cdot \nabla \mathbf{v}_{h}, \mathbf{u}_{\tau}^{n}\right)+B\left(\left(\mathbf{u}_{\tau}^{n}, p_{\tau}^{n}\right),\left(\mathbf{v}_{h}, q_{h}\right)\right)=0 .
\end{aligned}
$$

We shall prove (5.23) by considering the difference between (5.24), (5.25) and (5.20), (5.21). 
The proof is by mathematical induction. Note that the initial data satisfy

$$
\begin{aligned}
\left\|e_{\rho, h}^{0}\right\|_{L^{\infty}} & =\left\|P_{h} \rho^{0}-\Pi_{h} \rho^{0}\right\|_{L^{\infty}} \leq c h^{-1}\left\|P_{h} \rho^{0}-\Pi_{h} \rho^{0}\right\|_{L^{2}} \leq c\left\|\rho^{0}\right\|_{H^{2}} h, \\
\left\|\mathbf{e}_{\mathbf{u}, h}^{0}\right\|_{L^{2}} & =\left\|\mathbf{R}_{h}\left(\mathbf{u}^{0}, p^{0}\right)-\Pi_{h} \mathbf{u}^{0}\right\|_{L^{2}} \leq c\left(\left\|\mathbf{u}^{0}\right\|_{\mathbf{H}^{2}}+\left\|p^{0}\right\|_{H^{1}}\right) h, \\
\left\|\mathbf{e}_{\mathbf{u}, h}^{0}\right\|_{L^{\infty}} & =\left\|\mathbf{R}_{h}\left(\mathbf{u}^{0}, p^{0}\right)-\Pi_{h} \mathbf{u}^{0}\right\|_{L^{\infty}} \leq c h^{-1}\left\|\mathbf{R}_{h}\left(\mathbf{u}^{0}, p^{0}\right)-\Pi_{h} \mathbf{u}^{0}\right\|_{L^{2}} \leq c\left(\left\|\mathbf{u}^{0}\right\|_{\mathbf{H}^{2}}+\left\|p^{0}\right\|_{H^{1}}\right) h, \\
\tau\left\|\mathbf{e}_{\mathbf{u}, h}^{0}\right\|_{H^{1}}^{2} & \leq \tau\left\|\mathbf{R}_{h}\left(\mathbf{u}^{0}, p^{0}\right)-\Pi_{h} \mathbf{u}^{0}\right\|_{H^{1}}^{2} \leq c\left(\left\|\mathbf{u}^{0}\right\|_{H^{2}}^{2}+\left\|p^{0}\right\|_{H^{1}}^{2}\right) \tau h^{2} .
\end{aligned}
$$

For sufficiently small $\tau$ and $h$, the last four inequalities imply

$$
\begin{aligned}
\left\|e_{\rho, h}^{0}\right\|_{L^{\infty}} & \leq \frac{1}{4} \min _{x \in \bar{\Omega}} \rho^{0}(x), \\
\left\|\mathbf{e}_{\mathbf{u}, h}^{0}\right\|_{L^{2}} & \leq \sqrt{\tau+h^{2}} \\
\left\|\mathbf{e}_{\mathbf{u}, h}^{0}\right\|_{L^{\infty}} & \leq 1 \\
\tau\left\|\mathbf{e}_{\mathbf{u}, h}^{0}\right\|_{H^{1}}^{2} & \leq h^{2} \sqrt{\tau+h^{2}}
\end{aligned}
$$

Let $1 \leq m \leq N$, and assume that the data $\rho_{h}^{n-1}$ and $\mathbf{u}_{h}^{n-1}, n=1,2, \ldots, m$, are given and satisfying the following inequalities (induction assumption):

$$
\begin{aligned}
\max _{1 \leq n \leq m}\left\|e_{\rho, h}^{n-1}\right\|_{L^{\infty}} & \leq \frac{1}{4} \min _{x \in \bar{\Omega}} \rho^{0}(x), \\
\max _{1 \leq n \leq m}\left\|\mathbf{e}_{\mathbf{u}, h}^{n-1}\right\|_{L^{2}} & \leq h\left(\tau+h^{2}\right)^{\frac{1}{4}}, \\
\max _{1 \leq n \leq m}\left\|\mathbf{e}_{\mathbf{u}, h}^{n-1}\right\|_{L^{\infty}} & \leq 1 \\
\sum_{n=1}^{m} \tau\left\|\mathbf{e}_{\mathbf{u}, h}^{n-1}\right\|_{H^{1}}^{2} & \leq h^{2} \sqrt{\tau+h^{2}} .
\end{aligned}
$$

Then we prove that the solution $\left(\rho_{h}^{m}, \mathbf{u}_{h}^{m}, p_{h}^{m}\right) \in M_{h}^{2} \times \mathbf{X}_{h}^{2} \times \stackrel{\circ}{M}_{h}^{1}$ given by (5.20) and (5.21) satisfies the following inequalities:

$$
\begin{aligned}
\max _{0 \leq n \leq m}\left\|e_{\rho, h}^{n}\right\|_{L^{\infty}} & \leq \frac{1}{4} \min _{x \in \bar{\Omega}} \rho^{0}(x), \\
\max _{0 \leq n \leq m}\left\|\mathbf{e}_{\mathbf{u}, h}^{n}\right\|_{L^{2}} & \leq h\left(\tau+h^{2}\right)^{\frac{1}{4}} \\
\max _{0 \leq n \leq m}\left\|\mathbf{e}_{\mathbf{u}, h}^{n}\right\|_{L^{\infty}} & \leq 1 \\
\sum_{n=0}^{m} \tau\left\|\mathbf{e}_{\mathbf{u}, h}^{n}\right\|_{H^{1}}^{2} & \leq h^{2} \sqrt{\tau+h^{2}}
\end{aligned}
$$

To use mathematical induction, we emphasize that all the generic constants below will be independent of $m$ (but may depend on $T$ ).

Note that the induction assumption (5.29) implies that

$$
\begin{aligned}
\left\|\rho_{\tau}^{n-1}-\rho_{h}^{n-1}\right\|_{L^{\infty}} & \leq\left\|\rho_{\tau}^{n-1}-P_{h} \rho_{\tau}^{n-1}\right\|_{L^{\infty}}+\left\|e_{\rho, h}^{n-1}\right\|_{L^{\infty}} \\
& \leq c\left\|\rho_{\tau}^{n-1}\right\|_{W^{1, \infty}} h+\frac{1}{4} \min _{x \in \bar{\Omega}} \rho^{0}(x) \\
& \leq \frac{1}{2} \min _{x \in \bar{\Omega}} \rho^{0}(x) \quad \text { when } h \text { is sufficiently small }
\end{aligned}
$$


which further implies

$$
\begin{aligned}
& \rho_{h}^{n-1}(x) \geq \min _{x \in \bar{\Omega}} \rho^{0}(x)-\left\|\rho_{\tau}^{n-1}-\rho_{h}^{n-1}\right\|_{L^{\infty}} \geq \frac{1}{2} \min _{x \in \bar{\Omega}} \rho^{0}(x), \quad \forall x \in \Omega, \quad n=1, \ldots, m, \\
& \rho_{h}^{n-1}(x) \leq \max _{x \in \bar{\Omega}} \rho^{0}(x)+\left\|\rho_{\tau}^{n-1}-\rho_{h}^{n-1}\right\|_{L^{\infty}} \leq \frac{3}{2} \max _{x \in \bar{\Omega}} \rho^{0}(x), \quad \forall x \in \Omega, \quad n=1, \ldots, m,
\end{aligned}
$$

thus

$$
\frac{1}{2} \min _{y \in \bar{\Omega}} \rho^{0}(y) \leq \rho_{h}^{n-1} \leq \frac{3}{2} \max _{y \in \bar{\Omega}} \rho^{0}(y), \quad n=1, \ldots, m .
$$

Besides, the induction assumption (5.29) implies that

$$
\begin{aligned}
& \left\|\mathbf{u}_{h}^{n-1}\right\|_{L^{\infty}} \\
& \quad \leq\left\|\mathbf{R}_{h}\left(\mathbf{u}_{\tau}^{n-1}, p_{\tau}^{n-1}\right)\right\|_{L^{\infty}}+\left\|\mathbf{e}_{\mathbf{u}, h}^{n-1}\right\|_{L^{\infty}} \\
& \quad \leq\left\|\mathbf{R}_{h}\left(\mathbf{u}_{\tau}^{n-1}, p_{\tau}^{n-1}\right)-\Pi_{h} \mathbf{u}_{\tau}^{n-1}\right\|_{L^{\infty}}+\left\|\Pi_{h} \mathbf{u}_{\tau}^{n-1}-\mathbf{u}_{\tau}^{n-1}\right\|_{L^{\infty}}+\left\|\mathbf{u}_{\tau}^{n-1}\right\|_{L^{\infty}}+\left\|\mathbf{e}_{\mathbf{u}, h}^{n-1}\right\|_{L^{\infty}} \\
& \quad \leq c h^{-1}\left\|\mathbf{R}_{h}\left(\mathbf{u}_{\tau}^{n-1}, p_{\tau}^{n-1}\right)-\Pi_{h} \mathbf{u}_{\tau}^{n-1}\right\|_{L^{2}}+\left\|\Pi_{h} \mathbf{u}_{\tau}^{n-1}-\mathbf{u}_{\tau}^{n-1}\right\|_{L^{\infty}}+\left\|\mathbf{u}_{\tau}^{n-1}\right\|_{L^{\infty}}+\left\|\mathbf{e}_{\mathbf{u}, h}^{n-1}\right\|_{L^{\infty}} \\
& \quad \leq c\left(\left\|\mathbf{u}_{\tau}^{n-1}\right\|_{H^{2}}+\left\|p_{\tau}^{n-1}\right\|_{H^{1}}\right) h+c\left\|\mathbf{u}_{\tau}^{n-1}\right\|_{W^{1, \infty}} h+\left\|\mathbf{u}_{\tau}^{n-1}\right\|_{L^{\infty}}+1 \quad \text { (use (5.6), (5.15) and (5.29)) } \\
& \quad \leq c \quad \text { when } h \text { is sufficiently small, } \quad n=1, \ldots, m .
\end{aligned}
$$

The estimates (5.32) and (5.33) will be frequently used in the following subsections.

\subsection{Estimates of $e_{\rho, h}^{n}$}

Subtracting (5.20) from (5.24) yields

$$
\begin{aligned}
& \left(D_{\tau}\left(\rho_{\tau}^{n}-P_{h} \rho_{\tau}^{n}\right), \varphi_{h}\right)+\left(D_{\tau} e_{\rho, h}^{n}, \varphi_{h}\right) \\
& \quad+\left(\mathbf{u}_{h}^{n-1} \cdot \nabla\left(\rho_{\tau}^{n}-P_{h} \rho_{\tau}^{n}\right), \varphi_{h}\right)+\frac{1}{2}\left(\nabla \cdot \mathbf{u}_{h}^{n-1}\left(\rho_{\tau}^{n}-P_{h} \rho_{\tau}^{n}\right), \varphi_{h}\right) \\
& \quad+\left(\mathbf{u}_{h}^{n-1} \cdot \nabla e_{\rho, h}^{n}, \varphi_{h}\right)+\frac{1}{2}\left(\nabla \cdot \mathbf{u}_{h}^{n-1} e_{\rho, h}^{n}, \varphi_{h}\right) \\
& \quad+\left(\mathbf{e}_{\mathbf{u}, h}^{n-1} \cdot \nabla \rho_{\tau}^{n}, \varphi_{h}\right)+\frac{1}{2}\left(\nabla \cdot \mathbf{e}_{\mathbf{u}, h}^{n-1} \rho_{\tau}^{n}, \varphi_{h}\right) \\
& \quad+\left(\left(\mathbf{u}_{\tau}^{n-1}-\mathbf{R}_{h}\left(\mathbf{u}_{\tau}^{n-1}, p_{\tau}^{n-1}\right)\right) \cdot \nabla \rho_{\tau}^{n}, \varphi_{h}\right) \\
& \quad+\frac{1}{2}\left(\rho_{\tau}^{n} \nabla \cdot\left(\mathbf{u}_{\tau}^{n-1}-\mathbf{R}_{h}\left(\mathbf{u}_{\tau}^{n-1}, p_{\tau}^{n-1}\right)\right), \varphi_{h}\right)=0, \quad \forall \varphi_{h} \in M_{h}^{2} .
\end{aligned}
$$

Since $\left(D_{\tau}\left(\rho_{\tau}^{n}-P_{h} \rho_{\tau}^{n}\right), \varphi_{h}\right)=\left(D_{\tau} \rho_{\tau}^{n}-P_{h} D_{\tau} \rho_{\tau}^{n}, \varphi_{h}\right)=0$, taking $\varphi_{h}=e_{\rho, h}^{n}$ in the equation above yields

$$
\begin{aligned}
\frac{1}{2} D_{\tau}\left(\left\|e_{\rho, h}^{n}\right\|_{L^{2}}^{2}\right) \leq & \left|\left(\mathbf{u}_{h}^{n-1} \cdot \nabla\left(\rho_{\tau}^{n}-P_{h} \rho_{\tau}^{n}\right), e_{\rho, h}^{n}\right)\right| \\
& +\left|\frac{1}{2}\left(\nabla \cdot \mathbf{u}_{h}^{n-1}\left(\rho_{\tau}^{n}-P_{h} \rho_{\tau}^{n}\right), e_{\rho, h}^{n}\right)\right| \\
& +\left|\left(\mathbf{u}_{h}^{n-1} \cdot \nabla e_{\rho, h}^{n}, e_{\rho, h}^{n}\right)+\frac{1}{2}\left(\nabla \cdot \mathbf{u}_{h}^{n-1} e_{\rho, h}^{n}, e_{\rho, h}^{n}\right)\right| \\
& +\left|\left(\mathbf{e}_{\mathbf{u}, h}^{n-1} \cdot \nabla \rho_{\tau}^{n}, e_{\rho, h}^{n}\right)+\frac{1}{2}\left(\nabla \cdot \mathbf{e}_{\mathbf{u}, h}^{n-1} \rho_{\tau}^{n}, e_{\rho, h}^{n}\right)\right|
\end{aligned}
$$




$$
\begin{aligned}
& +\left|\left(\left(\mathbf{u}_{\tau}^{n-1}-\mathbf{R}_{h}\left(\mathbf{u}_{\tau}^{n-1}, p_{\tau}^{n-1}\right)\right) \cdot \nabla \rho_{\tau}^{n}, e_{\rho, h}^{n}\right)\right| \\
& +\left|\frac{1}{2}\left(\rho_{\tau}^{n} \nabla \cdot\left(\mathbf{u}_{\tau}^{n-1}-\mathbf{R}_{h}\left(\mathbf{u}_{\tau}^{n-1}, p_{\tau}^{n-1}\right)\right), e_{\rho, h}^{n}\right)\right| \\
= & : \sum_{k=1}^{6}\left|J_{k}\right|,
\end{aligned}
$$

where

$$
\begin{aligned}
& J_{1} \leq\left\|\mathbf{u}_{h}^{n-1}\right\|_{L^{\infty}}\left\|\nabla\left(\rho_{\tau}^{n}-P_{h} \rho_{\tau}^{n}\right)\right\|_{L^{2}}\left\|e_{\rho, h}^{n}\right\|_{L^{2}} \\
& \leq\left\|\mathbf{u}_{h}^{n-1}\right\|_{L^{\infty}}\left(c h \tau+c h^{2}\right)\left\|e_{\rho, h}^{n}\right\|_{L^{2}} \quad \text { (use (5.17)) } \\
& \leq\left(\operatorname{ch} \tau+c h^{2}\right)\left\|e_{\rho, h}^{n}\right\|_{L^{2}} \quad \text { (use (5.33)) } \\
& \leq c \epsilon^{-1} h^{2}\left(\tau^{2}+h^{2}\right)+\epsilon\left\|e_{\rho, h}^{n}\right\|_{L^{2}}^{2}, \quad \text { (use Hölder's inequality) } \\
& J_{2} \leq\left\|\nabla \mathbf{u}_{h}^{n-1}\right\|_{L^{\infty}}\left\|\rho_{\tau}^{n}-P_{h} \rho_{\tau}^{n}\right\|_{L^{2}}\left\|e_{\rho, h}^{n}\right\|_{L^{2}} \\
& \leq\left\|\nabla \mathbf{u}_{h}^{n-1}\right\|_{L^{\infty}}\left(c h^{2} \tau+c h^{3}\right)\left\|e_{\rho, h}^{n}\right\|_{L^{2}} \\
& \leq c\left\|\mathbf{u}_{h}^{n-1}\right\|_{L^{\infty}}\left(c h \tau+c h^{2}\right)\left\|e_{\rho, h}^{n}\right\|_{L^{2}}, \\
& \leq\left(\operatorname{ch} \tau+c h^{2}\right)\left\|e_{\rho, h}^{n}\right\|_{L^{2}}, \\
& \leq c \epsilon^{-1} h^{2}\left(\tau^{2}+h^{2}\right)+\epsilon\left\|e_{\rho, h}^{n}\right\|_{L^{2}}^{2} \\
& J_{3}=0, \\
& J_{4} \leq\left\|\mathbf{e}_{\mathbf{u}, h}^{n-1}\right\|_{L^{2}}\left\|\nabla \rho_{\tau}^{n}\right\|_{L^{\infty}}\left\|e_{\rho, h}^{n}\right\|_{L^{2}}+\left\|\nabla \mathbf{e}_{\mathbf{u}, h}^{n-1}\right\|_{L^{2}}\left\|\rho_{\tau}^{n}\right\|_{L^{\infty}}\left\|e_{\rho, h}^{n}\right\|_{L^{2}} \\
& \leq c\left(\left\|\mathbf{e}_{\mathbf{u}, h}^{n-1}\right\|_{L^{2}}+\left\|\nabla \mathbf{e}_{\mathbf{u}, h}^{n-1}\right\|_{L^{2}}\right)\left\|e_{\rho, h}^{n}\right\|_{L^{2}} \\
& \leq c \epsilon^{-1}\left(\left\|\mathbf{e}_{\mathbf{u}, h}^{n-1}\right\|_{L^{2}}^{2}+\left\|\nabla \mathbf{e}_{\mathbf{u}, h}^{n-1}\right\|_{L^{2}}^{2}\right)+\epsilon\left\|e_{\rho, h}^{n}\right\|_{L^{2}}^{2}, \\
& J_{5} \leq\left\|\mathbf{u}_{\tau}^{n-1}-\mathbf{R}_{h}\left(\mathbf{u}_{\tau}^{n-1}, p_{\tau}^{n-1}\right)\right\|_{L^{2}}\left\|\nabla \rho_{\tau}^{n}\right\|_{L^{\infty}}\left\|e_{\rho, h}^{n}\right\|_{L^{2}} \\
& \leq \operatorname{ch}^{2}\left(\left\|\mathbf{u}_{\tau}^{n-1}\right\|_{H^{2}}+\left\|p_{\tau}^{n-1}\right\|_{H^{1}}\right)\left\|\nabla \rho_{\tau}^{n}\right\|_{L^{\infty}}\left\|e_{\rho, h}^{n}\right\|_{L^{2}} \quad \text { (use (5.6)) } \\
& \leq c h^{2}\left\|e_{\rho, h}^{n}\right\|_{L^{2}} \\
& \leq c \epsilon^{-1} h^{4}+\epsilon\left\|e_{\rho, h}^{n}\right\|_{L^{2}}^{2}, \\
& J_{6} \leq\left\|\rho_{\tau}^{n}\right\|_{L^{\infty}}\left\|\nabla \cdot\left(\mathbf{u}_{\tau}^{n-1}-\mathbf{R}_{h}\left(\mathbf{u}_{\tau}^{n-1}, p_{\tau}^{n-1}\right)\right)\right\|_{L^{2}}\left\|e_{\rho, h}^{n}\right\|_{L^{2}} \\
& \leq\left\|\rho_{\tau}^{n}\right\|_{L^{\infty}}\left(\operatorname{ch} \tau^{\frac{1}{2}}+c h^{2}\right)\left\|e_{\rho, h}^{n}\right\|_{L^{2}} \\
& \leq\left(\operatorname{ch} \tau^{\frac{1}{2}}+c h^{2}\right)\left\|e_{\rho, h}^{n}\right\|_{L^{2}} \\
& \leq c \epsilon^{-1} h^{2}\left(\tau+h^{2}\right)+\epsilon\left\|e_{\rho, h}^{n}\right\|_{L^{2}}^{2} . \\
& \text { (use Hölder's inequality) }
\end{aligned}
$$

Substituting $J_{1}, \ldots, J_{6}$ into $(5.35)$ yields, for $n=1, \ldots, m$,

$$
D_{\tau}\left\|e_{\rho, h}^{n}\right\|_{L^{2}}^{2} \leq c \epsilon^{-1} h^{2}\left(\tau+h^{2}\right)+c \epsilon^{-1}\left(\left\|\mathbf{e}_{\mathbf{u}, h}^{n-1}\right\|_{L^{2}}^{2}+\left\|\nabla \mathbf{e}_{\mathbf{u}, h}^{n-1}\right\|_{L^{2}}^{2}\right)+\epsilon\left\|e_{\rho, h}^{n}\right\|_{L^{2}}^{2},
$$

which implies (by choosing a small $\epsilon$ and applying Grönwall's inequality) 


$$
\begin{aligned}
\max _{1 \leq n \leq k}\left\|e_{\rho, h}^{n}\right\|_{L^{2}}^{2} & \leq c h^{2}\left(\tau+h^{2}\right)+\sum_{n=1}^{k} c \tau\left\|\mathbf{e}_{\mathbf{u}, h}^{n-1}\right\|_{H^{1}}^{2}+c\left\|e_{\rho, h}^{0}\right\|_{L^{2}}^{2}, \\
& \leq c h^{2}\left(\tau+h^{2}\right)+\sum_{n=1}^{k} c \tau\left\|\mathbf{e}_{\mathbf{u}, h}^{n-1}\right\|_{H^{1}}^{2}+c\left\|P_{h} \rho^{0}-\Pi_{h} \rho^{0}\right\|_{L^{2}}^{2} \\
& \leq c h^{2}\left(\tau+h^{2}\right)+\sum_{n=1}^{k} c \tau\left\|\mathbf{e}_{\mathbf{u}, h}^{n-1}\right\|_{H^{1}}^{2} \quad k=1, \ldots, m .
\end{aligned}
$$

From the last inequality and the induction assumption (5.29), we derive

$$
\begin{aligned}
\max _{1 \leq n \leq k}\left\|e_{\rho, h}^{n}\right\|_{L^{2}} & \leq\left(c h^{2}\left(\tau+h^{2}\right)+\sum_{n=1}^{k} c \tau\left\|\mathbf{e}_{\mathbf{u}, h}^{n-1}\right\|_{H^{1}}^{2}\right)^{\frac{1}{2}} \leq c h\left(\tau+h^{2}\right)^{\frac{1}{4}} \\
\max _{1 \leq n \leq m}\left\|e_{\rho, h}^{n}\right\|_{L^{\infty}} & \leq c h^{-1} \max _{1 \leq n \leq m}\left\|e_{\rho, h}^{n}\right\|_{L^{2}} \\
& \leq c \sqrt{\tau+h^{2}}+c h^{-1}\left(\sum_{n=1}^{m} \tau\left\|\mathbf{e}_{\mathbf{u}, h}^{n-1}\right\|_{H^{1}}^{2}\right)^{\frac{1}{2}} \\
& \leq c\left(\tau+h^{2}\right)^{\frac{1}{4}}
\end{aligned}
$$

For sufficiently small $\tau$ and $h$ the last inequality implies

$$
\max _{1 \leq n \leq m}\left\|e_{\rho, h}^{n}\right\|_{L^{\infty}} \leq \frac{1}{4} \min _{x \in \bar{\Omega}} \rho^{0}(x) .
$$

Then the same argument as (5.31) and (5.32) shows that

$$
\frac{1}{2} \min _{x \in \bar{\Omega}} \rho^{0}(x) \leq \rho_{h}^{n}(x) \leq \frac{3}{2} \max _{x \in \bar{\Omega}} \rho^{0}(x), \quad \forall x \in \Omega, \quad n=1, \ldots, m .
$$

\subsection{Estimates of $D_{\tau} e_{\rho, h}^{\boldsymbol{n}}$}

We estimate $\left\|D_{\tau} e_{\rho, h}^{n}\right\|_{L^{2}}$ by taking $\varphi_{h}=D_{\tau} e_{\rho, h}^{n}$ in (5.34). Since

$$
\left(D_{\tau}\left(\rho_{\tau}^{n}-P_{h} \rho_{\tau}^{n}\right), D_{\tau} e_{\rho, h}^{n}\right)=\left(D_{\tau} \rho_{\tau}^{n}-P_{h} D_{\tau} \rho_{\tau}^{n}, D_{\tau} e_{\rho, h}^{n}\right)=0,
$$

we obtain

$$
\begin{aligned}
\left\|D_{\tau} e_{\rho, h}^{n}\right\|_{L^{2}} \leq & \left\|\mathbf{u}_{h}^{n-1} \cdot \nabla\left(\rho_{\tau}^{n}-P_{h} \rho_{\tau}^{n}\right)\right\|_{L^{2}}+\frac{1}{2}\left\|\nabla \cdot \mathbf{u}_{h}^{n-1}\left(\rho_{\tau}^{n}-P_{h} \rho_{\tau}^{n}\right)\right\|_{L^{2}} \\
& +\left\|\mathbf{u}_{h}^{n-1} \cdot \nabla e_{\rho, h}^{n}\right\|_{L^{2}}+\frac{1}{2}\left\|\nabla \cdot \mathbf{u}_{h}^{n-1} e_{\rho, h}^{n}\right\|_{L^{2}} \\
& +\left\|\mathbf{e}_{\mathbf{u}, h}^{n-1} \cdot \nabla \rho_{\tau}^{n}\right\|_{L^{2}}+\frac{1}{2}\left\|\nabla \cdot \mathbf{e}_{\mathbf{u}, h}^{n-1} \rho_{\tau}^{n}\right\|_{L^{2}} \\
& +\left\|\left(\mathbf{u}_{\tau}^{n-1}-\mathbf{R}_{h}\left(\mathbf{u}_{\tau}^{n-1}, p_{\tau}^{n-1}\right)\right) \cdot \nabla \rho_{\tau}^{n}\right\|_{L^{2}} \\
& +\frac{1}{2}\left\|\rho_{\tau}^{n} \nabla \cdot\left(\mathbf{u}_{\tau}^{n-1}-\mathbf{R}_{h}\left(\mathbf{u}_{\tau}^{n-1}, p_{\tau}^{n-1}\right)\right)\right\|_{L^{2}} \\
= & : \sum_{k=1}^{5} J_{k}^{*},
\end{aligned}
$$


where

$$
\begin{aligned}
& J_{1}^{*} \leq\left\|\mathbf{u}_{h}^{n-1}\right\|_{L^{\infty}} c h\left\|\rho_{\tau}^{n}\right\|_{H^{2}}+c\left\|\nabla \cdot \mathbf{u}_{h}^{n-1}\right\|_{L^{\infty}}\left\|\rho_{\tau}^{n}-P_{h} \rho_{\tau}^{n}\right\|_{L^{2}} \\
& \leq\left\|\mathbf{u}_{h}^{n-1}\right\|_{L^{\infty}} \operatorname{ch}\left\|\rho_{\tau}^{n}\right\|_{H^{2}}+c h^{-1}\left\|\mathbf{u}_{h}^{n-1}\right\|_{L^{\infty}} c h^{2}\left\|\rho_{\tau}^{n}\right\|_{H^{2}} \quad \text { (inverse inequality) } \\
& \leq \text { ch, } \quad \text { (use (3.9) to estimate }\left\|\rho_{\tau}^{n}\right\|_{H^{2}} \text {, and (5.33) to estimate }\left\|\mathbf{u}_{h}^{n-1}\right\|_{L^{\infty}} \text { ) } \\
& J_{2}^{*} \leq\left\|\mathbf{u}_{h}^{n-1}\right\|_{L^{\infty}}\left\|\nabla e_{\rho, h}^{n}\right\|_{L^{2}}+\left\|\nabla \cdot \mathbf{u}_{h}^{n-1}\right\|_{L^{\infty}}\left\|e_{\rho, h}^{n}\right\|_{L^{2}} \\
& \leq\left\|\mathbf{u}_{h}^{n-1}\right\|_{L^{\infty}} c h^{-1}\left\|e_{\rho, h}^{n}\right\|_{L^{2}}+c h^{-1}\left\|\mathbf{u}_{h}^{n-1}\right\|_{L^{\infty}}\left\|e_{\rho, h}^{n}\right\|_{L^{2}} \quad \text { (inverse inequality) } \\
& \leq\left\|\mathbf{u}_{h}^{n-1}\right\|_{L^{\infty}} c h^{-1} \operatorname{ch}\left(\tau+h^{2}\right)^{\frac{1}{4}}+c h^{-1}\left\|\mathbf{u}_{h}^{n-1}\right\|_{L^{\infty}} \operatorname{ch}\left(\tau+h^{2}\right)^{\frac{1}{4}} \\
& \text { (use (5.43) to estimate }\left\|e_{\rho, h}^{n}\right\|_{L^{2}} \text { ) } \\
& \leq c\left(\tau+h^{2}\right)^{\frac{1}{4}}, \quad\left(\text { use }(5.33) \text { to estimate }\left\|\mathbf{u}_{h}^{n-1}\right\|_{L^{\infty}}\right) \\
& J_{3}^{*} \leq\left\|\mathbf{e}_{\mathbf{u}, h}^{n-1}\right\|_{L^{2}}\left\|\nabla \rho_{\tau}^{n}\right\|_{L^{\infty}}+\left\|\nabla \cdot \mathbf{e}_{\mathbf{u}, h}^{n-1}\right\|_{L^{2}}\left\|\rho_{\tau}^{n}\right\|_{L^{\infty}} \\
& \leq\left\|\mathbf{e}_{\mathbf{u}, h}^{n-1}\right\|_{L^{2}} \mid\left\|\nabla \rho_{\tau}^{n}\right\|_{L^{\infty}}+c h^{-1}\left\|\mathbf{e}_{\mathbf{u}, h}^{n-1}\right\|_{L^{2}}\left\|\rho_{\tau}^{n}\right\|_{L^{\infty}} \quad \text { (inverse inequality) } \\
& \leq \operatorname{ch}\left(\tau+h^{2}\right)^{\frac{1}{4}}\left\|\nabla \rho_{\tau}^{n}\right\|_{L^{\infty}}+c h^{-1} \operatorname{ch}\left(\tau+h^{2}\right)^{\frac{1}{4}}\left\|\rho_{\tau}^{n}\right\|_{L^{\infty}} \\
& \text { (use (5.29) to estimate }\left\|\mathbf{e}_{\mathbf{u}, h}^{n-1}\right\|_{L^{2}} \text { ) } \\
& \left.\leq c\left(\tau+h^{2}\right)^{\frac{1}{4}}, \quad \text { (use (3.9) to estimate }\left\|\nabla \rho_{\tau}^{n}\right\|_{L^{\infty}} \text { and }\left\|\rho_{\tau}^{n}\right\|_{L^{\infty}}\right) \\
& J_{4}^{*} \leq c h^{2}\left(\left\|\mathbf{u}_{\tau}^{n-1}\right\|_{H^{2}}+\left\|p_{\tau}^{n-1}\right\|_{H^{1}}\right)\left\|\nabla \rho_{\tau}^{n}\right\|_{L^{\infty}} \leq c h^{2}, \quad \text { (use (3.9)) } \\
& J_{5}^{*} \leq c\left\|\rho_{\tau}^{n}\right\|_{L^{\infty}}\left\|\nabla \cdot\left(\mathbf{u}_{\tau}^{n-1}-\mathbf{R}_{h}\left(\mathbf{u}_{\tau}^{n-1}, p_{\tau}^{n-1}\right)\right)\right\|_{L^{2}} \\
& \leq c\left\|\rho_{\tau}^{n}\right\|_{L^{\infty}} \operatorname{ch}\left(\left\|\mathbf{u}_{\tau}^{n-1}\right\|_{H^{2}}+\left\|p_{\tau}^{n-1}\right\|_{H^{1}}\right) \quad \text { (inverse inequality) } \\
& \leq \text { ch. } \quad \text { (use (3.9) to estimate }\left\|\rho_{\tau}^{n}\right\|_{L^{\infty}},\left\|\mathbf{u}_{\tau}^{n-1}\right\|_{H^{2}} \text { and }\left\|p_{\tau}^{n-1}\right\|_{H^{1}} \text { ) }
\end{aligned}
$$

Substituting (5.48)-(5.52) into (5.47) yields

$$
\left\|D_{\tau} e_{\rho, h}^{n}\right\|_{L^{2}} \leq c\left(\tau+h^{2}\right)^{\frac{1}{4}} .
$$

\subsection{Estimates of $\mathrm{e}_{\mathrm{u}, h}^{n}$}

By using integration by parts, equation (5.21) can be rewritten as

$$
\begin{aligned}
\left(\rho_{h}^{n-1} D_{\tau} \mathbf{u}_{h}^{n}, v_{h}\right) & +\frac{1}{2}\left(D_{\tau} \rho_{h}^{n} \mathbf{u}_{h}^{n}, v_{h}\right)+\frac{1}{2}\left(\rho_{h}^{n} \mathbf{u}_{h}^{n-1} \cdot \nabla \mathbf{u}_{h}^{n}, \mathbf{v}_{h}\right)-\frac{1}{2}\left(\rho_{h}^{n} \mathbf{u}_{h}^{n-1} \cdot \nabla \mathbf{v}_{h}, \mathbf{u}_{h}^{n}\right) \\
& +B\left(\left(\mathbf{u}_{h}^{n}, p_{h}^{n}\right),\left(\mathbf{v}_{h}, q_{h}\right)\right)=0 .
\end{aligned}
$$

Subtracting (5.54) from (5.26), we obtain

$$
\begin{aligned}
& {\left[\left(\rho_{h}^{n-1} D_{\tau} \mathbf{e}_{\mathbf{u}, h}^{n}, \mathbf{v}_{h}\right)+\left(\rho_{h}^{n-1} D_{\tau}\left(\mathbf{u}_{\tau}^{n}-\mathbf{R}_{h}\left(\mathbf{u}_{\tau}^{n}, p_{\tau}^{n}\right)\right), \mathbf{v}_{h}\right)\right.} \\
& \left.\quad+\left(\left(\rho_{\tau}^{n-1}-P_{h} \rho_{\tau}^{n-1}\right) D_{\tau} \mathbf{u}_{\tau}^{n}, \mathbf{v}_{h}\right)+\left(e_{\rho, h}^{n-1} D_{\tau} \mathbf{u}_{\tau}^{n}, \mathbf{v}_{h}\right)\right] \\
& \quad+\frac{1}{2}\left[\left(D_{\tau} \rho_{\tau}^{n} \mathbf{e}_{\mathbf{u}, h}^{n}, \mathbf{v}_{h}\right)+\left(D_{\tau} \rho_{\tau}^{n}\left(\mathbf{u}_{\tau}^{n}-\mathbf{R}_{h}\left(\mathbf{u}_{\tau}^{n}, p_{\tau}^{n}\right)\right), \mathbf{v}_{h}\right)\right. \\
& \left.\quad+\left(D_{\tau}\left(\rho_{\tau}^{n}-P_{h} \rho_{\tau}^{n}\right) \mathbf{u}_{h}^{n}, \mathbf{v}_{h}\right)+\left(D_{\tau} e_{\rho, h}^{n} \mathbf{u}_{h}^{n}, \mathbf{v}_{h}\right)\right] \\
& \quad+\frac{1}{2}\left[\left(\rho_{h}^{n} \mathbf{u}_{h}^{n-1} \nabla \mathbf{e}_{\mathbf{u}, h}^{n}, \mathbf{v}_{h}\right)+\left(\rho_{h}^{n} \mathbf{u}_{h}^{n-1} \nabla\left(\mathbf{u}_{\tau}^{n}-\mathbf{R}_{h}\left(\mathbf{u}_{\tau}^{n}, p_{\tau}^{n}\right)\right), \mathbf{v}_{h}\right)\right. \\
& \quad+\left(\rho_{h}^{n}\left(\mathbf{u}_{\tau}^{n-1}-\mathbf{R}_{h}\left(\mathbf{u}_{\tau}^{n-1}, p_{\tau}^{n-1}\right)\right) \cdot \nabla \mathbf{u}_{\tau}^{n}, \mathbf{v}_{h}\right)+\left(\rho_{h}^{n} \mathbf{e}_{\mathbf{u}, h}^{n-1} \cdot \nabla \mathbf{u}_{\tau}^{n}, \mathbf{v}_{h}\right)
\end{aligned}
$$




$$
\begin{aligned}
& \left.+\left(\left(\rho_{\tau}^{n}-P_{h} \rho_{\tau}^{n}\right) \mathbf{u}_{\tau}^{n-1} \cdot \nabla \mathbf{u}_{\tau}^{n}, \mathbf{v}_{h}\right)+\left(e_{\rho, h}^{n} \mathbf{u}_{\tau}^{n-1} \cdot \nabla \mathbf{u}_{\tau}^{n}, \mathbf{v}_{h}\right)\right] \\
& -\frac{1}{2}\left[\left(\rho_{h}^{n} \mathbf{u}_{h}^{n-1} \cdot \nabla \mathbf{v}_{h}, \mathbf{e}_{\mathbf{u}, h}^{n}\right)+\left(\rho_{h}^{n} \mathbf{u}_{h}^{n-1} \cdot \nabla \mathbf{v}_{h}, \mathbf{u}_{\tau}^{n}-\mathbf{R}_{h}\left(\mathbf{u}_{\tau}^{n}, p_{\tau}^{n}\right)\right)\right. \\
& +\left(\rho_{h}^{n}\left(\mathbf{u}_{\tau}^{n-1}-\mathbf{R}_{h}\left(\mathbf{u}_{\tau}^{n-1}, p_{\tau}^{n-1}\right)\right) \cdot \nabla \mathbf{v}_{h}, \mathbf{u}_{\tau}^{n}\right)+\left(\rho_{h}^{n} \mathbf{e}_{\mathbf{u}, h}^{n-1} \cdot \nabla \mathbf{v}_{h}, \mathbf{u}_{\tau}^{n}\right) \\
& \left.+\left(\left(\rho_{\tau}^{n}-P_{h} \rho_{\tau}^{n}\right) \mathbf{u}_{\tau}^{n-1} \cdot \nabla \mathbf{v}_{h}, \mathbf{u}_{\tau}^{n}\right)+\left(e_{\rho, h}^{n} \mathbf{u}_{\tau}^{n-1} \cdot \nabla \mathbf{v}_{h}, \mathbf{u}_{\tau}^{n}\right)\right] \\
& +B\left(\left(\mathbf{e}_{\mathbf{u}, h}^{n}, e_{p, h}^{n}\right),\left(\mathbf{v}_{h}, q_{h}\right)\right) \\
& =0 .
\end{aligned}
$$

Reformulating the last equation yields

$$
\begin{aligned}
&\left(\rho_{h}^{n-1} D_{\tau} \mathbf{e}_{\mathbf{u}, h}^{n}, \mathbf{v}_{h}\right)+\frac{1}{2}\left(D_{\tau} \rho_{h}^{n} \mathbf{e}_{\mathbf{u}, h}^{n}, \mathbf{v}_{h}\right)+B\left(\left(\mathbf{e}_{\mathbf{u}, h}^{n}, e_{p, h}^{n}\right),\left(\mathbf{v}_{h}, q_{h}\right)\right) \\
&=-\left(\rho_{h}^{n-1} D_{\tau}\left(\mathbf{u}_{\tau}^{n}-\mathbf{R}_{h}\left(\mathbf{u}_{\tau}^{n}, p_{\tau}^{n}\right)\right), \mathbf{v}_{h}\right) \\
&- {\left[\left(\left(\rho_{\tau}^{n-1}-P_{h} \rho_{\tau}^{n-1}\right) D_{\tau} \mathbf{u}_{\tau}^{n}, \mathbf{v}_{h}\right)+\left(e_{\rho, h}^{n-1} D_{\tau} \mathbf{u}_{\tau}^{n}, \mathbf{v}_{h}\right)\right] } \\
&-\frac{1}{2}\left(D_{\tau} e_{\rho, h}^{n} \mathbf{e}_{\mathbf{u}, h}^{n}, \mathbf{v}_{h}\right)-\frac{1}{2}\left(D_{\tau}\left(\rho_{\tau}^{n}-P_{h} \rho_{\tau}^{n}\right) \mathbf{e}_{\mathbf{u}, h}^{n}, \mathbf{v}_{h}\right)-\frac{1}{2}\left(D_{\tau} \rho_{\tau}^{n}\left(\mathbf{u}_{\tau}^{n}-\mathbf{R}_{h}\left(\mathbf{u}_{\tau}^{n}, p_{\tau}^{n}\right)\right), \mathbf{v}_{h}\right) \\
&-\frac{1}{2}\left[\left(D_{\tau}\left(\rho_{\tau}^{n}-P_{h} \rho_{\tau}^{n}\right) \mathbf{u}_{h}^{n}, \mathbf{v}_{h}\right)+\left(D_{\tau} e_{\rho, h}^{n} \mathbf{u}_{h}^{n}, \mathbf{v}_{h}\right)\right] \\
&-\frac{1}{2}\left[\left(\rho_{h}^{n} \mathbf{u}_{h}^{n-1} \nabla \mathbf{e}_{\mathbf{u}, h}^{n}, \mathbf{v}_{h}\right)+\left(\rho_{h}^{n} \mathbf{u}_{h}^{n-1} \nabla\left(\mathbf{u}_{\tau}^{n}-\mathbf{R}_{h}\left(\mathbf{u}_{\tau}^{n}, p_{\tau}^{n}\right)\right), \mathbf{v}_{h}\right)\right] \\
&-\frac{1}{2}\left[\left(\rho_{h}^{n}\left(\mathbf{u}_{\tau}^{n-1}-\mathbf{R}_{h}\left(\mathbf{u}_{\tau}^{n-1}, p_{\tau}^{n-1}\right)\right) \cdot \nabla \mathbf{u}_{\tau}^{n}, \mathbf{v}_{h}\right)+\left(\rho_{h}^{n} \mathbf{e}_{\mathbf{u}, h}^{n-1} \cdot \nabla \mathbf{u}_{\tau}^{n}, \mathbf{v}_{h}\right)\right] \\
&-\frac{1}{2}\left[\left(\left(\rho_{\tau}^{n}-P_{h} \rho_{\tau}^{n}\right) \mathbf{u}_{\tau}^{n-1} \cdot \nabla \mathbf{u}_{\tau}^{n}, \mathbf{v}_{h}\right)+\left(e_{\rho, h}^{n} \mathbf{u}_{\tau}^{n-1} \cdot \nabla \mathbf{u}_{\tau}^{n}, \mathbf{v}_{h}\right)\right] \\
&+\frac{1}{2}\left[\left(\rho_{h}^{n} \mathbf{u}_{h}^{n-1} \cdot \nabla \mathbf{v}_{h}, \mathbf{e}_{\mathbf{u}, h}^{n}\right)+\left(\rho_{h}^{n} \mathbf{u}_{h}^{n-1} \cdot \nabla \mathbf{v}_{h}, \mathbf{u}_{\tau}^{n}-\mathbf{R}_{h}\left(\mathbf{u}_{\tau}^{n}, p_{\tau}^{n}\right)\right)\right] \\
&+\frac{1}{2}\left[\left(\rho_{h}^{n}\left(\mathbf{u}_{\tau}^{n-1}-\mathbf{R}_{h}\left(\mathbf{u}_{\tau}^{n-1}, p_{\tau}^{n-1}\right)\right) \cdot \nabla \mathbf{v}_{h}, \mathbf{u}_{\tau}^{n}\right)+\left(\rho_{h}^{n} \mathbf{e}_{\mathbf{u}, h}^{n-1} \cdot \nabla \mathbf{v}_{h}, \mathbf{u}_{\tau}^{n}\right)\right] \\
&+\frac{1}{2}\left[\left(\left(\rho_{\tau}^{n}-P_{h} \rho_{\tau}^{n}\right) \mathbf{u}_{\tau}^{n-1} \cdot \nabla \mathbf{v}_{h}, \mathbf{u}_{\tau}^{n}\right)+\left(e_{\rho, h}^{n} \mathbf{u}_{\tau}^{n-1} \cdot \nabla \mathbf{v}_{h}, \mathbf{u}_{\tau}^{n}\right)\right] \\
&= \sum_{j=1}^{10} I_{j}^{*}\left(\mathbf{v}_{h}\right) .
\end{aligned}
$$

Substituting $\left(\mathbf{v}_{h}, q_{h}\right)=\left(\mathbf{e}_{\mathbf{u}, h}^{n}, e_{p, h}^{n}\right)$ and using the interpolation inequality

$$
\begin{aligned}
\left\|\mathbf{e}_{\mathbf{u}, h}^{n}\right\|_{L^{4}}^{2} & \leq c\left\|\mathbf{e}_{\mathbf{u}, h}^{n}\right\|_{H^{\frac{1}{2}}}^{2} & & \text { (Sobolev embedding, cf. [51], Eq. (32.7)) } \\
& \leq c\left\|\mathbf{e}_{\mathbf{u}, h}^{n}\right\|_{L^{2}}\left\|\mathbf{e}_{\mathbf{u}, h}^{n}\right\|_{H^{1}}, & & \text { (interpolation inequality, cf. [51], Lem. 25.2) }
\end{aligned}
$$

we have

$$
\begin{aligned}
\left|I_{1}^{*}\left(\mathbf{e}_{\mathbf{u}, h}^{n}\right)\right| & =\left|\left(\rho_{h}^{n-1} D_{\tau}\left(\mathbf{u}_{\tau}^{n}-\mathbf{R}_{h}\left(\mathbf{u}_{\tau}^{n}, p_{\tau}^{n}\right)\right), \mathbf{e}_{\mathbf{u}, h}^{n}\right)\right| \\
& \leq\left\|\rho_{h}^{n-1}\right\|_{L^{\infty}}\left\|D_{\tau} \mathbf{u}_{\tau}^{n}-\mathbf{R}_{h}\left(D_{\tau} \mathbf{u}_{\tau}^{n}, D_{\tau} p_{\tau}^{n}\right)\right\|_{L^{2}}\left\|\mathbf{e}_{\mathbf{u}, h}^{n}\right\|_{L^{2}} \\
& \leq c\left(\left\|D_{\tau} \mathbf{u}_{\tau}^{n}\right\|_{H^{2}}+\left\|D_{\tau} p_{\tau}^{n}\right\|_{H^{1}}\right) h^{2}\left\|\mathbf{e}_{\mathbf{u}, h}^{n}\right\|_{L^{2}} \\
& \leq c \epsilon^{-1}\left(\left\|D_{\tau} \mathbf{u}_{\tau}^{n}\right\|_{H^{2}}^{2}+\left\|D_{\tau} p_{\tau}^{n}\right\|_{H^{1}}^{2}\right) h^{4}+\epsilon\left\|\mathbf{e}_{\mathbf{u}, h}^{n}\right\|_{L^{2}}^{2}
\end{aligned}
$$




$$
\begin{aligned}
\left|I_{2}^{*}\left(\mathbf{e}_{\mathbf{u}, h}^{n}\right)\right| & =\left|\left(\left(\rho_{\tau}^{n-1}-P_{h} \rho_{\tau}^{n-1}\right) D_{\tau} \mathbf{u}_{\tau}^{n}, \mathbf{e}_{\mathbf{u}, h}^{n}\right)+\left(e_{\rho, h}^{n-1} D_{\tau} \mathbf{u}_{\tau}^{n}, \mathbf{e}_{\mathbf{u}, h}^{n}\right)\right| \\
& \leq\left\|D_{\tau} \mathbf{u}_{\tau}^{n}\right\|_{L^{\infty}}\left\|\mathbf{e}_{\mathbf{u}, h}^{n}\right\|_{L^{2}}\left(\left\|\rho_{\tau}^{n-1}-P_{h} \rho_{\tau}^{n-1}\right\|_{L^{2}}+\left\|e_{\rho, h}^{n-1}\right\|_{L^{2}}\right) \\
& \leq\left\|D_{\tau} \mathbf{u}_{\tau}^{n}\right\|_{L^{\infty}}\left\|\mathbf{e}_{\mathbf{u}, h}^{n}\right\|_{L^{2}}\left(h^{2}\left\|\rho_{\tau}^{n-1}\right\|_{H^{2}}+\left\|e_{\rho, h}^{n-1}\right\|_{L^{2}}\right) \\
& \left.\leq c\left\|\mathbf{e}_{\mathbf{u}, h}^{n}\right\|_{L^{2}}\left(h^{2}+\left\|e_{\rho, h}^{n-1}\right\|_{L^{2}}\right) \quad \text { (use (3.9) to control }\left\|\rho_{\tau}^{n-1}\right\|_{H^{2}} \text { and }\left\|D_{\tau} \mathbf{u}_{\tau}^{n}\right\|_{L^{\infty}}\right) \\
& \leq c \epsilon^{-1}\left(h^{4}+\left\|e_{\rho, h}^{n-1}\right\|_{L^{2}}^{2}\right)+\epsilon\left\|\mathbf{e}_{\mathbf{u}, h}^{n}\right\|_{L^{2}}^{2},
\end{aligned}
$$

$$
\begin{aligned}
\left|I_{3}^{*}\left(\mathbf{e}_{\mathbf{u}, h}^{n}\right)\right| \leq & c\left\|D_{\tau} e_{\rho, h}^{n}\right\|_{L^{2}}\left\|\mathbf{e}_{\mathbf{u}, h}^{n}\right\|_{L^{4}}^{2}+c\left\|D_{\tau}\left(\rho_{\tau}^{n}-P_{h} \rho_{\tau}^{n}\right)\right\|_{L^{2}}\left\|\mathbf{e}_{\mathbf{u}, h}^{n}\right\|_{L^{4}}^{2} \\
& +c\left\|D_{\tau} \rho_{\tau}^{n}\right\|_{L^{\infty}}\left\|\mathbf{u}_{\tau}^{n}-\mathbf{R}_{h}\left(\mathbf{u}_{\tau}^{n}, p_{\tau}^{n}\right)\right\|_{L^{2}}\left\|\mathbf{e}_{\mathbf{u}, h}^{n}\right\|_{L^{2}} \\
\leq & c\left(\tau+h^{2}\right)^{\frac{1}{4}}\left\|\mathbf{e}_{\mathbf{u}, h}^{n}\right\|_{L^{2}}\left\|\nabla \mathbf{e}_{\mathbf{u}, h}^{n}\right\|_{L^{2}}+c h^{2}\left\|D_{\tau} \rho_{\tau}^{n}\right\|_{H^{2}}\left\|\mathbf{e}_{\mathbf{u}, h}^{n}\right\|_{L^{2}}\left\|\nabla \mathbf{e}_{\mathbf{u}, h}^{n}\right\|_{L^{2}}
\end{aligned}
$$$$
+c\left\|D_{\tau} \rho_{\tau}^{n}\right\|_{L^{\infty}} h^{2}\left(\left\|\mathbf{u}_{\tau}^{n}\right\|_{H^{2}}+\left\|p_{\tau}^{n}\right\|_{H^{1}}\right)\left\|\mathbf{e}_{\mathbf{u}, h}^{n}\right\|_{L^{2}} \quad \text { (use (5.6)) }
$$$$
\leq c\left(\tau+h^{2}\right)^{\frac{1}{4}}\left\|\mathbf{e}_{\mathbf{u}, h}^{n}\right\|_{L^{2}}^{2}+c\left(\tau+h^{2}\right)^{\frac{1}{4}}\left\|\nabla \mathbf{e}_{\mathbf{u}, h}^{n}\right\|_{L^{2}}^{2}+c\left\|\mathbf{e}_{\mathbf{u}, h}^{n}\right\|_{L^{2}}^{2}+c h^{4}
$$

(use (3.9) to control $\left\|\mathbf{u}_{\tau}^{n}\right\|_{H^{2}}$ and $\left\|D_{\tau} \rho_{\tau}^{n}\right\|_{L^{\infty}}$ )

$\left|I_{5}^{*}\left(\mathbf{e}_{\mathbf{u}, h}^{n}\right)\right| \leq\left|\left(\rho_{h}^{n} \mathbf{u}_{h}^{n-1} \nabla \mathbf{e}_{\mathbf{u}, h}^{n}, \mathbf{e}_{\mathbf{u}, h}^{n}\right)+\left(\rho_{h}^{n} \mathbf{u}_{h}^{n-1} \nabla\left(\mathbf{u}_{\tau}^{n}-\mathbf{R}_{h}\left(\mathbf{u}_{\tau}^{n}, p_{\tau}^{n}\right)\right), \mathbf{e}_{\mathbf{u}, h}^{n}\right)\right|$

$$
\begin{aligned}
& \leq c\left\|\rho_{h}^{n}\right\|_{L^{\infty}}\left\|\mathbf{u}_{h}^{n-1}\right\|_{L^{\infty}}\left(\left\|\nabla \mathbf{e}_{\mathbf{u}, h}^{n}\right\|_{L^{2}}+\left\|\nabla\left(\mathbf{u}_{\tau}^{n}-\mathbf{R}_{h}\left(\mathbf{u}_{\tau}^{n}, p_{\tau}^{n}\right)\right)\right\|_{L^{2}}\right)\left\|\mathbf{e}_{\mathbf{u}, h}^{n}\right\|_{L^{2}} \\
& \leq c\left(\left\|\nabla \mathbf{e}_{\mathbf{u}, h}^{n}\right\|_{L^{2}}+\left\|\nabla\left(\mathbf{u}_{\tau}^{n}-\mathbf{R}_{h}\left(\mathbf{u}_{\tau}^{n}, p_{\tau}^{n}\right)\right)\right\|_{L^{2}}\right)\left\|\mathbf{e}_{\mathbf{u}, h}^{n}\right\|_{L^{2}} \quad \text { (use (5.33) and (5.46)) }
\end{aligned}
$$$$
\leq\left(c\left\|\nabla \mathbf{e}_{\mathbf{u}, h}^{n}\right\|_{L^{2}}+c h \tau^{\frac{1}{2}}+c h^{2}\right)\left\|\mathbf{e}_{\mathbf{u}, h}^{n}\right\|_{L^{2}} \quad \text { (use (5.19)) }
$$

$$
\begin{aligned}
& \leq \epsilon\left\|\nabla \mathbf{e}_{\mathbf{u}, h}^{n}\right\|_{L^{2}}^{2}+c \epsilon^{-1}\left\|\mathbf{e}_{\mathbf{u}, h}^{n}\right\|_{L^{2}}^{2}+c \epsilon^{-1} h^{2}\left(\tau+h^{2}\right), \\
\left|I_{6}^{*}\left(\mathbf{e}_{\mathbf{u}, h}^{n}\right)\right| & \leq\left\|\rho_{h}^{n}\right\|_{L^{\infty}}\left(\left\|\mathbf{u}_{\tau}^{n-1}-\mathbf{R}_{h}\left(\mathbf{u}_{\tau}^{n-1}, p_{\tau}^{n-1}\right)\right\|_{L^{2}}+\left\|\mathbf{e}_{\mathbf{u}, h}^{n-1}\right\|_{L^{2}}\right)\left\|\nabla \mathbf{u}_{\tau}^{n}\right\|_{L^{\infty}}\left\|\mathbf{e}_{\mathbf{u}, h}^{n}\right\|_{L^{2}}
\end{aligned}
$$$$
\left.\leq c\left(\left\|\mathbf{u}_{\tau}^{n-1}-\mathbf{R}_{h}\left(\mathbf{u}_{\tau}^{n-1}, p_{\tau}^{n-1}\right)\right\|_{L^{2}}+\left\|\mathbf{e}_{\mathbf{u}, h}^{n-1}\right\|_{L^{2}}\right)\left\|\mathbf{e}_{\mathbf{u}, h}^{n}\right\|_{L^{2}} \quad \text { (use (3.9) for }\left\|\nabla \mathbf{u}_{\tau}^{n}\right\|_{L^{\infty}}\right)
$$

$\leq c\left(h^{2}\left(\left\|\mathbf{u}_{\tau}^{n-1}\right\|_{H^{2}}+\left\|p_{\tau}^{n-1}\right\|_{H^{1}}\right)+\left\|\mathbf{e}_{\mathbf{u}, h}^{n-1}\right\|_{L^{2}}\right)\left\|\mathbf{e}_{\mathbf{u}, h}^{n}\right\|_{L^{2}} \quad$ (use (5.6)) $\leq c\left(h^{2}+\left\|\mathbf{e}_{\mathbf{u}, h}^{n-1}\right\|_{L^{2}}\right)\left\|\mathbf{e}_{\mathbf{u}, h}^{n}\right\|_{L^{2}} \quad$ (use (3.9) to control $\left\|\mathbf{u}_{\tau}^{n-1}\right\|_{H^{2}}$ and $\left\|p_{\tau}^{n-1}\right\|_{H^{1}}$ )

$$
\leq c h^{4}+c\left(\left\|\mathbf{e}_{\mathbf{u}, h}^{n-1}\right\|_{L^{2}}^{2}+\left\|\mathbf{e}_{\mathbf{u}, h}^{n}\right\|_{L^{2}}^{2}\right),
$$

$$
\begin{aligned}
\left|I_{7}^{*}\left(\mathbf{e}_{\mathbf{u}, h}^{n}\right)\right| & \leq\left(\left\|\rho_{\tau}^{n}-P_{h} \rho_{\tau}^{n}\right\|_{L^{2}}+\left\|e_{\rho, h}^{n}\right\|_{L^{2}}\right)\left\|\mathbf{u}_{\tau}^{n-1}\right\|_{L^{\infty}}\left\|\nabla \mathbf{u}_{\tau}^{n}\right\|_{L^{\infty}}\left\|\mathbf{e}_{\mathbf{u}, h}^{n}\right\|_{L^{2}} \\
& \leq\left(c h^{2}\left\|\rho_{\tau}^{n}\right\|_{H^{2}}+\left\|e_{\rho, h}^{n}\right\|_{L^{2}}\right)\left\|\mathbf{u}_{\tau}^{n-1}\right\|_{L^{\infty}}\left\|\nabla \mathbf{u}_{\tau}^{n}\right\|_{L^{\infty}}\left\|\mathbf{e}_{\mathbf{u}, h}^{n}\right\|_{L^{2}} \quad \text { (use (5.10)) } \\
& \left.\leq c\left(h^{2}+\left\|e_{\rho, h}^{n}\right\|_{L^{2}}\right)\left\|\mathbf{e}_{\mathbf{u}, h}^{n}\right\|_{L^{2}} \quad \text { (use (3.9) to control }\left\|\rho_{\tau}^{n}\right\|_{H^{2}} \text { and }\left\|\nabla \mathbf{u}_{\tau}^{n}\right\|_{L^{\infty}}\right) \\
& \leq c h^{4}+c\left(\left\|e_{\rho, h}^{n}\right\|_{L^{2}}^{2}+\left\|\mathbf{e}_{\mathbf{u}, h}^{n}\right\|_{L^{2}}^{2}\right),
\end{aligned}
$$

$$
\begin{aligned}
\left|I_{8}^{*}\left(\mathbf{e}_{\mathbf{u}, h}^{n}\right)\right| & \leq\left|\left(\rho_{h}^{n} \mathbf{u}_{h}^{n-1} \cdot \nabla \mathbf{e}_{\mathbf{u}, h}^{n}, \mathbf{e}_{\mathbf{u}, h}^{n}\right)+\left(\rho_{h}^{n} \mathbf{u}_{h}^{n-1} \cdot \nabla \mathbf{e}_{\mathbf{u}, h}^{n}, \mathbf{u}_{\tau}^{n}-\mathbf{R}_{h}\left(\mathbf{u}_{\tau}^{n}, p_{\tau}^{n}\right)\right)\right| \\
& \leq\left\|\rho_{h}^{n}\right\|_{L^{\infty}}\left\|\mathbf{u}_{h}^{n-1}\right\|_{L^{\infty}}\left\|\nabla \mathbf{e}_{\mathbf{u}, h}^{n}\right\|_{L^{2}}\left(\left\|\mathbf{e}_{\mathbf{u}, h}^{n}\right\|_{L^{2}}+\| \mathbf{u}_{\tau}^{n}-\mathbf{R}_{h}\left(\mathbf{u}_{\tau}^{n}, p_{\tau}^{n}\right)\right) \|_{L^{2}} \\
& \leq\left\|\rho_{h}^{n}\right\|_{L^{\infty}}\left\|\mathbf{u}_{h}^{n-1}\right\|_{L^{\infty}}\left\|\nabla \mathbf{e}_{\mathbf{u}, h}^{n}\right\|_{L^{2}}\left(\left\|\mathbf{e}_{\mathbf{u}, h}^{n}\right\|_{L^{2}}+c h^{2}\left(\left\|\mathbf{u}_{\tau}^{n-1}\right\|_{H^{2}}+\left\|p_{\tau}^{n-1}\right\|_{H^{1}}\right)\right)
\end{aligned}
$$




$$
\begin{aligned}
\leq c\left\|\nabla \mathbf{e}_{\mathbf{u}, h}^{n}\right\|_{L^{2}}\left(\left\|\mathbf{e}_{\mathbf{u}, h}^{n}\right\|_{L^{2}}+h^{2}\right) \quad \text { (use (5.33) and (5.46) to control }\left\|\rho_{h}^{n}\right\|_{L^{\infty}} \|_{\left.\mathbf{u}_{h}^{n-1} \|_{L^{\infty}}\right)} & \left.\quad \text { use (3.9) to control }\left\|\mathbf{u}_{\tau}^{n-1}\right\|_{H^{2}} \text { and }\left\|p_{\tau}^{n-1}\right\|_{H^{1}}\right) \\
& \leq \epsilon\left\|\nabla \mathbf{e}_{\mathbf{u}, h}^{n}\right\|_{L^{2}}^{2}+c \epsilon^{-1}\left(\left\|\mathbf{e}_{\mathbf{u}, h}^{n}\right\|_{L^{2}}^{2}+h^{4}\right) \\
\left|I_{9}^{*}\left(\mathbf{e}_{\mathbf{u}, h}^{n}\right)\right| & \leq\left|\left(\rho_{h}^{n}\left(\mathbf{u}_{\tau}^{n-1}-\mathbf{R}_{h}\left(\mathbf{u}_{\tau}^{n-1}, p_{\tau}^{n-1}\right)\right) \cdot \nabla \mathbf{e}_{\mathbf{u}, h}^{n}, \mathbf{u}_{\tau}^{n}\right)+\left(\rho_{h}^{n} \mathbf{e}_{\mathbf{u}, h}^{n-1} \cdot \nabla \mathbf{e}_{\mathbf{u}, h}^{n}, \mathbf{u}_{\tau}^{n}\right)\right| \\
& \leq\left\|\rho_{h}^{n}\right\|_{L^{\infty}}\left(\| \mathbf{u}_{\tau}^{n-1}-\mathbf{R}_{h}\left(\mathbf{u}_{\tau}^{n-1}, p_{\tau}^{n-1}\right)\right)\left\|_{L^{2}}+\right\| \mathbf{e}_{\mathbf{u}, h}^{n-1}\left\|_{L^{2}}\right\| \nabla \mathbf{e}_{\mathbf{u}, h}^{n-1}\left\|_{L^{2}}\right\| \mathbf{u}_{\tau}^{n} \|_{L^{\infty}} \\
& \leq\left\|\rho_{h}^{n}\right\|_{L^{\infty}}\left(c h^{2}\left(\left\|\mathbf{u}_{\tau}^{n-1}\right\|_{H^{2}}+\left\|p_{\tau}^{n-1}\right\|_{H^{1}}\right)+\left\|\mathbf{e}_{\mathbf{u}, h}^{n-1}\right\|_{L^{2}}\right)\left\|\nabla \mathbf{e}_{\mathbf{u}, h}^{n-1}\right\|_{L^{2}}\left\|\mathbf{u}_{\tau}^{n}\right\|_{L^{\infty}} \\
& \leq\left(c h^{2}+\left\|\mathbf{e}_{\mathbf{u}, h}^{n-1}\right\|_{L^{2}}\right)\left\|\nabla \mathbf{e}_{\mathbf{u}, h}^{n-1}\right\|_{L^{2}} \\
& \leq \epsilon\left\|\nabla \mathbf{e}_{\mathbf{u}, h}^{n-1}\right\|_{L^{2}}^{2}+c \epsilon^{-1}\left(\left\|\mathbf{e}_{\mathbf{u}, h}^{n-1}\right\|_{L^{2}}^{2}+h^{4}\right) \\
\left|I_{10}^{*}\left(\mathbf{e}_{\mathbf{u}, h}^{n}\right)\right| & \leq\left|\left(\left(\rho_{\tau}^{n}-P_{h} \rho_{\tau}^{n}\right) \mathbf{u}_{\tau}^{n-1} \cdot \nabla \mathbf{e}_{\mathbf{u}, h}^{n}, \mathbf{u}_{\tau}^{n}\right)+\left(e_{\rho, h}^{n} \mathbf{u}_{\tau}^{n-1} \cdot \nabla \mathbf{e}_{\mathbf{u}, h}^{n}, \mathbf{u}_{\tau}^{n}\right)\right| \\
& \leq\left(\left\|\rho_{\tau}^{n}-P_{h} \rho_{\tau}^{n}\right\|_{L^{2}}+\left\|e_{\rho, h}^{n}\right\|_{L^{2}}\right)\left\|\mathbf{u}_{\tau}^{n-1}\right\|_{L^{\infty}}\left\|\nabla \mathbf{e}_{\mathbf{u}, h}^{n}\right\|_{L^{2}}\left\|\mathbf{u}_{\tau}^{n}\right\|_{L^{\infty}} \\
& \leq\left(c h^{2}\left\|\rho_{\tau}^{n}\right\|_{H^{2}}+\left\|e_{\rho, h}^{n}\right\|_{L^{2}}\right)\left\|\mathbf{u}_{\tau}^{n-1}\right\|_{L^{\infty}}\left\|\nabla \mathbf{e}_{\mathbf{u}, h}^{n}\right\|_{L^{2}}\left\|\mathbf{u}_{\tau}^{n}\right\|_{L^{\infty}} \\
& \leq\left(c h^{2}+\left\|e_{\rho, h}^{n}\right\|_{L^{2}}\right)\left\|\nabla \mathbf{e}_{\mathbf{u}, h}^{n}\right\|_{L^{2}} \\
& \leq \epsilon\left\|\nabla \mathbf{e}_{\mathbf{u}, h}^{n}\right\|_{L^{2}}^{2}+c \epsilon^{-1}\left(h^{4}+\left\|e_{\rho, h}^{n}\right\|_{L^{2}}^{2}\right) .
\end{aligned}
$$

It remains to estimate $\left|I_{4}^{*}\left(\mathbf{e}_{\mathbf{u}, h}^{n}\right)\right|$. To this end, we substitute $\varphi_{h}=P_{h}\left(\mathbf{u}_{h}^{n} \cdot \mathbf{e}_{\mathbf{u}, h}^{n}\right)$ into (5.34). Then we obtain

$$
\begin{aligned}
\left|\left(D_{\tau} e_{\rho, h}^{n}, P_{h}\left(\mathbf{u}_{h}^{n} \cdot \mathbf{e}_{\mathbf{u}, h}^{n}\right)\right)\right| \leq & \left|\left(\mathbf{u}_{h}^{n-1} \cdot \nabla\left(\rho_{\tau}^{n}-P_{h} \rho_{\tau}^{n}\right), P_{h}\left(\mathbf{u}_{h}^{n} \cdot \mathbf{e}_{\mathbf{u}, h}^{n}\right)\right)\right| \\
& +\frac{1}{2}\left|\left(\nabla \cdot \mathbf{u}_{h}^{n-1}\left(\rho_{\tau}^{n}-P_{h} \rho_{\tau}^{n}\right), P_{h}\left(\mathbf{u}_{h}^{n} \cdot \mathbf{e}_{\mathbf{u}, h}^{n}\right)\right)\right| \\
& +\left|\left(\mathbf{u}_{h}^{n-1} \cdot \nabla e_{\rho, h}^{n}, P_{h}\left(\mathbf{u}_{h}^{n} \cdot \mathbf{e}_{\mathbf{u}, h}^{n}\right)\right)\right| \\
& +\frac{1}{2}\left|\left(\nabla \cdot \mathbf{u}_{h}^{n-1} e_{\rho, h}^{n}, P_{h}\left(\mathbf{u}_{h}^{n} \cdot \mathbf{e}_{\mathbf{u}, h}^{n}\right)\right)\right| \\
& +\left|\left(\mathbf{e}_{\mathbf{u}, h}^{n-1} \cdot \nabla \rho_{\tau}^{n}, P_{h}\left(\mathbf{u}_{h}^{n} \cdot \mathbf{e}_{\mathbf{u}, h}^{n}\right)\right)\right| \\
& +\frac{1}{2}\left|\left(\nabla \cdot \mathbf{e}_{\mathbf{u}, h}^{n-1} \rho_{\tau}^{n}, P_{h}\left(\mathbf{u}_{h}^{n} \cdot \mathbf{e}_{\mathbf{u}, h}^{n}\right)\right)\right| \\
& +\left|\left(\left(\mathbf{u}_{\tau}^{n-1}-\mathbf{R}_{h}\left(\mathbf{u}_{\tau}^{n-1}, p_{\tau}^{n-1}\right)\right) \cdot \nabla \rho_{\tau}^{n}, P_{h}\left(\mathbf{u}_{h}^{n} \cdot \mathbf{e}_{\mathbf{u}, h}^{n}\right)\right)\right| \\
& +\frac{1}{2}\left|\left(\rho_{\tau}^{n} \nabla \cdot\left(\mathbf{u}_{\tau}^{n-1}-\mathbf{R}_{h}\left(\mathbf{u}_{\tau}^{n-1}, p_{\tau}^{n-1}\right)\right), P_{h}\left(\mathbf{u}_{h}^{n} \cdot \mathbf{e}_{\mathbf{u}, h}^{n}\right)\right)\right| \\
= & : \sum_{i=1}^{8} H_{i} .
\end{aligned}
$$

By the decomposition $\mathbf{u}_{h}^{n} \cdot \mathbf{e}_{\mathbf{u}, h}^{n}=\left(\mathbf{R}_{h}\left(\mathbf{u}_{\tau}^{n}, p_{\tau}^{n}\right)-\mathbf{e}_{\mathbf{u}, h}^{n}\right) \cdot \mathbf{e}_{\mathbf{u}, h}^{n}=\mathbf{R}_{h}\left(\mathbf{u}_{\tau}^{n}, p_{\tau}^{n}\right) \cdot \mathbf{e}_{\mathbf{u}, h}^{n}-\left|\mathbf{e}_{\mathbf{u}, h}^{n}\right|^{2}$, we have

$$
\begin{aligned}
H_{1} & \leq\left|\left(\mathbf{u}_{h}^{n-1} \cdot \nabla\left(\rho_{\tau}^{n}-P_{h} \rho_{\tau}^{n}\right), P_{h}\left(\left|\mathbf{e}_{\mathbf{u}, h}^{n}\right|^{2}\right)\right)\right|+\left|\left(\mathbf{u}_{h}^{n-1} \cdot \nabla\left(\rho_{\tau}^{n}-P_{h} \rho_{\tau}^{n}\right), P_{h}\left(\mathbf{R}_{h}\left(\mathbf{u}_{\tau}^{n}, p_{\tau}^{n}\right) \cdot \mathbf{e}_{\mathbf{u}, h}^{n}\right)\right)\right| \\
& \leq c\left\|\mathbf{u}_{h}^{n-1}\right\|_{L^{\infty}}\left\|\nabla\left(\rho_{\tau}^{n}-P_{h} \rho_{\tau}^{n}\right)\right\|_{L^{2}}\left(\left\|\mathbf{e}_{\mathbf{u}, h}^{n}\right\|_{L^{4}}^{2}+\left\|\mathbf{R}_{h}\left(\mathbf{u}_{\tau}^{n}, p_{\tau}^{n}\right)\right\|_{L^{\infty}}\left\|\mathbf{e}_{\mathbf{u}, h}^{n}\right\|_{L^{2}}\right) \\
& \leq c\left\|\mathbf{u}_{h}^{n-1}\right\|_{L^{\infty}}\left(c h \tau+c h^{2}\right)\left(\left\|\mathbf{e}_{\mathbf{u}, h}^{n}\right\|_{L^{4}}^{2}+\left\|\mathbf{e}_{\mathbf{u}, h}^{n}\right\|_{L^{2}}\right) \quad \text { (use (5.17) and (5.8)) } \quad \text { (use (5.33) and (5.57)) } \\
& \leq\left(c h \tau+c h^{2}\right)\left(\left\|\nabla \mathbf{e}_{\mathbf{u}, h}^{n}\right\|_{L^{2}}\left\|\mathbf{e}_{\mathbf{u}, h}^{n}\right\|_{L^{2}}+\left\|\mathbf{e}_{\mathbf{u}, h}^{n}\right\|_{L^{2}}\right) \\
& \leq c h(\tau+h)\left(\left\|\nabla \mathbf{e}_{\mathbf{u}, h}^{n}\right\|_{L^{2}}^{2}+\left\|\mathbf{e}_{\mathbf{u}, h}^{n}\right\|_{L^{2}}^{2}\right)+c h^{2}\left(\tau^{2}+h^{2}\right)+c\left\|\mathbf{e}_{\mathbf{u}, h}^{n}\right\|_{L^{2}}^{2}
\end{aligned}
$$




$$
\begin{aligned}
H_{2} & \leq\left|\left(\nabla \cdot \mathbf{u}_{h}^{n-1}\left(\rho_{\tau}^{n}-P_{h} \rho_{\tau}^{n}\right), P_{h}\left(\left|\mathbf{e}_{\mathbf{u}, h}^{n}\right|^{2}\right)\right)\right|+\left|\left(\nabla \cdot \mathbf{u}_{h}^{n-1}\left(\rho_{\tau}^{n}-P_{h} \rho_{\tau}^{n}\right), P_{h}\left(\mathbf{R}_{h}\left(\mathbf{u}_{\tau}^{n}, p_{\tau}^{n}\right) \cdot \mathbf{e}_{\mathbf{u}, h}^{n}\right)\right)\right| \\
& \leq\left\|\nabla \mathbf{u}_{h}^{n-1}\right\|_{L^{\infty}}\left\|\rho_{\tau}^{n}-P_{h} \rho_{\tau}^{n}\right\|_{L^{2}}\left(\left\|\mathbf{e}_{\mathbf{u}, h}^{n}\right\|_{L^{4}}^{2}+\left\|\mathbf{R}_{h}\left(\mathbf{u}_{\tau}^{n}, p_{\tau}^{n}\right)\right\|_{L^{\infty}}\left\|\mathbf{e}_{\mathbf{u}, h}^{n}\right\|_{L^{2}}\right) \\
& \leq\left\|\nabla \mathbf{u}_{h}^{n-1}\right\|_{L^{\infty}}\left(c h^{2} \tau+c h^{3}\right)\left(\left\|\mathbf{e}_{\mathbf{u}, h}^{n}\right\|_{L^{4}}^{2}+\left\|\mathbf{e}_{\mathbf{u}, h}^{n}\right\|_{L^{2}}\right) \quad(\text { use (5.16)) } \\
& \leq\left\|\mathbf{u}_{h}^{n-1}\right\|_{L^{\infty}}\left(c h \tau+c h^{2}\right)\left(\left\|\nabla \mathbf{e}_{\mathbf{u}, h}^{n}\right\|_{L^{2}}\left\|\mathbf{e}_{\mathbf{u}, h}^{n}\right\|_{L^{2}}+\left\|\mathbf{e}_{\mathbf{u}, h}^{n}\right\|_{L^{2}}\right)
\end{aligned}
$$$$
\leq\left(\operatorname{ch} \tau+c h^{2}\right)\left(\left\|\nabla \mathbf{e}_{\mathbf{u}, h}^{n}\right\|_{L^{2}}\left\|\mathbf{e}_{\mathbf{u}, h}^{n}\right\|_{L^{2}}+\left\|\mathbf{e}_{\mathbf{u}, h}^{n}\right\|_{L^{2}}\right)
$$

$$
\begin{aligned}
& \leq \operatorname{ch}(\tau+h)\left(\left\|\nabla \mathbf{e}_{\mathbf{u}, h}^{n}\right\|_{L^{2}}^{2}+\left\|\mathbf{e}_{\mathbf{u}, h}^{n}\right\|_{L^{2}}^{2}\right)+c h^{2}\left(\tau^{2}+h^{2}\right)+c\left\|\mathbf{e}_{\mathbf{u}, h}^{n}\right\|_{L^{2}}^{2} \\
H_{3} & \leq\left|\left(\mathbf{u}_{h}^{n-1} \cdot \nabla e_{\rho, h}^{n}, P_{h}\left(\left|\mathbf{e}_{\mathbf{u}, h}^{n}\right|^{2}\right)\right)\right|+\left|\left(\mathbf{u}_{h}^{n-1} \cdot \nabla e_{\rho, h}^{n}, P_{h}\left(\mathbf{R}_{h}\left(\mathbf{u}_{\tau}^{n}, p_{\tau}^{n}\right) \cdot \mathbf{e}_{\mathbf{u}, h}^{n}\right)\right)\right| \\
& =\left|\left(e_{\rho, h}^{n}, \nabla \cdot\left(\mathbf{u}_{h}^{n-1} P_{h}\left(\left|\mathbf{e}_{\mathbf{u}, h}^{n}\right|^{2}\right)\right)\right)\right|+\left|\left(e_{\rho, h}^{n}, \nabla \cdot\left(\mathbf{u}_{h}^{n-1} P_{h}\left(\mathbf{R}_{h}\left(\mathbf{u}_{\tau}^{n}, p_{\tau}^{n}\right) \cdot \mathbf{e}_{\mathbf{u}, h}^{n}\right)\right)\right)\right|
\end{aligned}
$$

$$
=\left|\left(e_{\rho, h}^{n}, \nabla \cdot \mathbf{u}_{h}^{n-1} P_{h}\left(\left|\mathbf{e}_{\mathbf{u}, h}^{n}\right|^{2}\right)\right)+\left(e_{\rho, h}^{n}, \mathbf{u}_{h}^{n-1} \cdot \nabla P_{h}\left(\left|\mathbf{e}_{\mathbf{u}, h}^{n}\right|^{2}\right)\right)\right|
$$$$
+\left|\left(e_{\rho, h}^{n}, \nabla \cdot \mathbf{u}_{h}^{n-1} P_{h}\left(\mathbf{R}_{h}\left(\mathbf{u}_{\tau}^{n}, p_{\tau}^{n}\right) \cdot \mathbf{e}_{\mathbf{u}, h}^{n}\right)\right)+\left(e_{\rho, h}^{n}, \mathbf{u}_{h}^{n-1} \cdot \nabla P_{h}\left(\mathbf{R}_{h}\left(\mathbf{u}_{\tau}^{n}, p_{\tau}^{n}\right) \cdot \mathbf{e}_{\mathbf{u}, h}^{n}\right)\right)\right|
$$$$
\leq c\left\|e_{\rho, h}^{n}\right\|_{L^{\infty}}\left\|\nabla \cdot \mathbf{u}_{h}^{n-1}\right\|_{L^{\infty}}\left\|\mathbf{e}_{\mathbf{u}, h}^{n}\right\|_{L^{2}}^{2}+c\left\|e_{\rho, h}^{n}\right\|_{L^{\infty}}\left\|\mathbf{u}_{h}^{n-1}\right\|_{L^{\infty}}\left\|\nabla\left|\mathbf{e}_{\mathbf{u}, h}^{n}\right|^{2}\right\|_{L^{1}}
$$

$$
+c\left\|e_{\rho, h}^{n}\right\|_{L^{2}}\left\|\nabla \cdot \mathbf{u}_{h}^{n-1}\right\|_{L^{\infty}}\left\|\mathbf{R}_{h}\left(\mathbf{u}_{\tau}^{n}, p_{\tau}^{n}\right) \cdot \mathbf{e}_{\mathbf{u}, h}^{n}\right\|_{L^{2}}
$$

$$
\begin{aligned}
& +c\left\|e_{\rho, h}^{n}\right\|_{L^{2}}\left\|\mathbf{u}_{h}^{n-1}\right\|_{L^{\infty}}\left\|\nabla\left(\mathbf{R}_{h}\left(\mathbf{u}_{\tau}^{n}, p_{\tau}^{n}\right) \cdot \mathbf{e}_{\mathbf{u}, h}^{n}\right)\right\|_{L^{2}} \\
\leq & c\left\|\nabla \mathbf{u}_{h}^{n-1}\right\|_{L^{\infty}}\left\|\mathbf{e}_{\mathbf{u}, h}^{n}\right\|_{L^{2}}^{2}+c\left\|\nabla \mathbf{e}_{\mathbf{u}, h}^{n}\right\|_{L^{2}}\left\|\mathbf{e}_{\mathbf{u}, h}^{n}\right\|_{L^{2}}
\end{aligned}
$$$$
+c\left\|\nabla \mathbf{u}_{h}^{n-1}\right\|_{L^{\infty}}\left\|e_{\rho, h}^{n}\right\|_{L^{2}}\left\|\mathbf{e}_{\mathbf{u}, h}^{n}\right\|_{L^{2}}+c\left\|e_{\rho, h}^{n}\right\|_{L^{2}}\left\|\nabla \mathbf{e}_{\mathbf{u}, h}^{n}\right\|_{L^{2}}
$$$$
\leq c\left\|\nabla \mathbf{u}_{h}^{n-1}\right\|_{L^{\infty}}\left(\left\|\mathbf{e}_{\mathbf{u}, h}^{n}\right\|_{L^{2}}^{2}+\left\|e_{\rho, h}^{n}\right\|_{L^{2}}^{2}\right)+c \epsilon^{-1}\left(\left\|\mathbf{e}_{\mathbf{u}, h}^{n}\right\|_{L^{2}}^{2}+\left\|e_{\rho, h}^{n}\right\|_{L^{2}}^{2}\right)+\epsilon\left\|\nabla \mathbf{e}_{\mathbf{u}, h}^{n}\right\|_{L^{2}}^{2},
$$

where the last inequality uses $\left\|\mathbf{R}_{h}\left(\mathbf{u}_{\tau}^{n}, p_{\tau}^{n}\right)\right\|_{W^{1, \infty}}$, which is a consequence of (5.8) and (3.9). Since (5.8) implies

$$
\begin{aligned}
\left\|\nabla \mathbf{u}_{h}^{n-1}\right\|_{L^{\infty}} & \leq\left\|\nabla \mathbf{e}_{\mathbf{u}, h}^{n-1}\right\|_{L^{\infty}}+\left\|\nabla \mathbf{R}_{h}\left(\mathbf{u}_{\tau}^{n-1}, p_{\tau}^{n-1}\right)\right\|_{L^{\infty}} \\
& \leq\left\|\nabla \mathbf{e}_{\mathbf{u}, h}^{n-1}\right\|_{L^{\infty}}+c \\
& \leq c h^{-1}\left\|\nabla \mathbf{e}_{\mathbf{u}, h}^{n-1}\right\|_{L^{2}}+c
\end{aligned}
$$

where the last step is due to the inverse inequality. Substituting the last inequality into the estimate of $H_{3}$ above, we obtain

$$
H_{3} \leq c\left(h^{-1}\left\|\nabla \mathbf{e}_{\mathbf{u}, h}^{n-1}\right\|_{L^{2}}+1\right)\left(\left\|\mathbf{e}_{\mathbf{u}, h}^{n}\right\|_{L^{2}}^{2}+\left\|e_{\rho, h}^{n}\right\|_{L^{2}}^{2}\right)+c \epsilon^{-1}\left(\left\|\mathbf{e}_{\mathbf{u}, h}^{n}\right\|_{L^{2}}^{2}+\left\|e_{\rho, h}^{n}\right\|_{L^{2}}^{2}\right)+\epsilon\left\|\nabla \mathbf{e}_{\mathbf{u}, h}^{n}\right\|_{L^{2}}^{2} .
$$

Similarly, we have

$$
\begin{aligned}
H_{4} & \leq\left|\left(e_{\rho, h}^{n} \nabla \cdot \mathbf{u}_{h}^{n-1}, P_{h}\left(\left|\mathbf{e}_{\mathbf{u}, h}^{n}\right|^{2}\right)\right)\right|+\left|\left(e_{\rho, h}^{n} \nabla \cdot \mathbf{u}_{h}^{n-1}, P_{h}\left(\mathbf{R}_{h}\left(\mathbf{u}_{\tau}^{n}, p_{\tau}^{n}\right) \mathbf{e}_{\mathbf{u}, h}^{n}\right)\right)\right| \\
& \leq c\left\|\nabla \mathbf{u}_{h}^{n-1}\right\|_{L^{\infty}}\left(\left\|e_{\rho, h}^{n}\right\|_{L^{\infty}}\left\|\mathbf{e}_{\mathbf{u}, h}^{n}\right\|_{L^{2}}^{2}+\left\|e_{\rho, h}^{n}\right\|_{L^{2}}\left\|\mathbf{e}_{\mathbf{u}, h}^{n}\right\|_{L^{2}}\right) \\
& \leq c\left(h^{-1}\left\|\nabla \mathbf{e}_{\mathbf{u}, h}^{n-1}\right\|_{L^{2}}+1\right)\left(\left\|\mathbf{e}_{\mathbf{u}, h}^{n}\right\|_{L^{2}}^{2}+\left\|e_{\rho, h}^{n}\right\|_{L^{2}}^{2}\right),
\end{aligned}
$$




$$
\begin{aligned}
& H_{5} \leq\left|\left(\mathbf{e}_{\mathbf{u}, h}^{n-1} \cdot \nabla \rho_{\tau}^{n}, P_{h}\left(\left|\mathbf{e}_{\mathbf{u}, h}^{n}\right|^{2}\right)\right)\right|+\left|\left(\mathbf{e}_{\mathbf{u}, h}^{n-1} \cdot \nabla \rho_{\tau}^{n}, P_{h}\left(\mathbf{R}_{h}\left(\mathbf{u}_{\tau}^{n}, p_{\tau}^{n}\right) \mathbf{e}_{\mathbf{u}, h}^{n}\right)\right)\right| \\
& \leq c\left\|\nabla \rho_{\tau}^{n}\right\|_{L^{\infty}}\left(\left\|\mathbf{e}_{\mathbf{u}, h}^{n-1}\right\|_{L^{\infty}}\left\|\mathbf{e}_{\mathbf{u}, h}^{n}\right\|_{L^{2}}^{2}+\left\|\mathbf{e}_{\mathbf{u}, h}^{n-1}\right\|_{L^{2}}\left\|\mathbf{e}_{\mathbf{u}, h}^{n}\right\|_{L^{2}}\right) \\
& \leq c\left(\left\|\mathbf{e}_{\mathbf{u}, h}^{n}\right\|_{L^{2}}^{2}+\left\|\mathbf{e}_{\mathbf{u}, h}^{n-1}\right\|_{L^{2}}^{2}\right) \text {, } \\
& H_{6} \leq\left|\left(\rho_{\tau}^{n} \nabla \cdot \mathbf{e}_{\mathbf{u}, h}^{n-1}, P_{h}\left(\left|\mathbf{e}_{\mathbf{u}, h}^{n}\right|^{2}\right)\right)\right|+\left|\left(\rho_{\tau}^{n} \nabla \cdot \mathbf{e}_{\mathbf{u}, h}^{n-1}, P_{h}\left(\mathbf{R}_{h}\left(\mathbf{u}_{\tau}^{n}, p_{\tau}^{n}\right) \mathbf{e}_{\mathbf{u}, h}^{n}\right)\right)\right| \\
& \leq c\left\|\rho_{\tau}^{n}\right\|_{L^{\infty}}\left(\left\|\nabla \mathbf{e}_{\mathbf{u}, h}^{n-1}\right\|_{L^{\infty}}\left\|\mathbf{e}_{\mathbf{u}, h}^{n}\right\|_{L^{2}}^{2}+\left\|\nabla \mathbf{e}_{\mathbf{u}, h}^{n-1}\right\|_{L^{2}}\left\|\mathbf{e}_{\mathbf{u}, h}^{n}\right\|_{L^{2}}\right) \\
& \leq c\left\|\rho_{\tau}^{n}\right\|_{L^{\infty}}\left(h^{-1}\left\|\nabla \mathbf{e}_{\mathbf{u}, h}^{n-1}\right\|_{L^{2}}\left\|\mathbf{e}_{\mathbf{u}, h}^{n}\right\|_{L^{2}}^{2}+\left\|\nabla \mathbf{e}_{\mathbf{u}, h}^{n-1}\right\|_{L^{2}}\left\|\mathbf{e}_{\mathbf{u}, h}^{n}\right\|_{L^{2}}\right) \\
& \leq c h^{-1}\left\|\nabla \mathbf{e}_{\mathbf{u}, h}^{n-1}\right\|_{L^{2}}\left\|\mathbf{e}_{\mathbf{u}, h}^{n}\right\|_{L^{2}}^{2}+\epsilon\left\|\nabla \mathbf{e}_{\mathbf{u}, h}^{n-1}\right\|_{L^{2}}^{2}+c \epsilon^{-1}\left\|\mathbf{e}_{\mathbf{u}, h}^{n}\right\|_{L^{2}}^{2}, \\
& H_{7} \leq\left|\left(\mathbf{u}_{\tau}^{n-1}-\mathbf{R}_{h}\left(\mathbf{u}_{\tau}^{n-1}, p_{\tau}^{n-1}\right)\right) \cdot \nabla \rho_{\tau}^{n}, P_{h}\left(\left|\mathbf{e}_{\mathbf{u}, h}^{n}\right|^{2}\right)-P_{h}\left(\mathbf{R}_{h}\left(\mathbf{u}_{\tau}^{\mathbf{n}}, p_{\tau}^{n}\right) \mathbf{e}_{\mathbf{u}, h}^{n}\right)\right| \\
& \leq\left\|\mathbf{u}_{\tau}^{n-1}-\mathbf{R}_{h}\left(\mathbf{u}_{\tau}^{n-1}, p_{\tau}^{n-1}\right)\right\|_{L^{2}}\left\|\nabla \rho_{\tau}^{n}\right\|_{L^{\infty}}\left(\left\|\mathbf{e}_{\mathbf{u}, h}^{n}\right\|_{L^{4}}^{2}+\left\|\mathbf{e}_{\mathbf{u}, h}^{n}\right\|_{L^{2}}\right) \\
& \leq \operatorname{ch}^{2}\left(\left\|\mathbf{u}_{\tau}^{n-1}\right\|_{H^{2}}+\left\|p_{\tau}^{n-1}\right\|_{H^{1}}\right)\left\|\nabla \rho_{\tau}^{n}\right\|_{L^{\infty}}\left(\left\|\nabla \mathbf{e}_{\mathbf{u}, h}^{n}\right\|_{L^{2}}\left\|\mathbf{e}_{\mathbf{u}, h}^{n}\right\|_{L^{2}}+\left\|\mathbf{e}_{\mathbf{u}, h}^{n}\right\|_{L^{2}}\right) \\
& \leq c h^{2}\left(\left\|\nabla \mathbf{e}_{\mathbf{u}, h}^{n}\right\|_{L^{2}}\left\|\mathbf{e}_{\mathbf{u}, h}^{n}\right\|_{L^{2}}+\left\|\mathbf{e}_{\mathbf{u}, h}^{n}\right\|_{L^{2}}\right) \\
& \leq c h^{2}\left(\left\|\nabla \mathbf{e}_{\mathbf{u}, h}^{n}\right\|_{L^{2}}^{2}+\left\|\mathbf{e}_{\mathbf{u}, h}^{n}\right\|_{L^{2}}^{2}\right)+\epsilon\left\|\mathbf{e}_{\mathbf{u}, h}^{n}\right\|_{L^{2}}^{2}+c h^{4}, \\
& H_{8} \leq\left|\left(\rho_{\tau}^{n} \nabla \cdot\left(\mathbf{u}^{n-1}-\mathbf{R}_{h}\left(\mathbf{u}_{\tau}^{n-1}, p_{\tau}^{n-1}\right)\right), P_{h}\left(\left|\mathbf{e}_{\mathbf{u}, h}^{n}\right|^{2}\right)-P_{h}\left(\mathbf{R}_{h}\left(\mathbf{u}_{\tau}^{n}, p_{\tau}^{n}\right) \mathbf{e}_{\mathbf{u}, h}^{n}\right)\right)\right| \\
& \leq\left\|\rho_{\tau}^{n}\right\|_{L^{\infty}}\left\|\nabla\left(\mathbf{u}_{\tau}^{n-1}-\mathbf{R}_{h}\left(\mathbf{u}_{\tau}^{n-1}, p_{\tau}^{n-1}\right)\right)\right\|_{L^{2}}\left(\left\|\mathbf{e}_{\mathbf{u}, h}^{n}\right\|_{L^{4}}^{2}+\left\|\mathbf{e}_{\mathbf{u}, h}^{n}\right\|_{L^{2}}\right) \\
& \leq\left\|\rho_{\tau}^{n}\right\|_{L^{\infty}}\left(\operatorname{ch} \tau^{\frac{1}{2}}+c h^{2}\right)\left(\left\|\mathbf{e}_{\mathbf{u}, h}^{n}\right\|_{L^{4}}^{2}+\left\|\mathbf{e}_{\mathbf{u}, h}^{n}\right\|_{L^{2}}\right) \\
& \leq\left(\operatorname{ch\tau ^{\frac {1}{2}}}+c h^{2}\right)\left(\left\|\nabla \mathbf{e}_{\mathbf{u}, h}^{n}\right\|_{L^{2}}\left\|\mathbf{e}_{\mathbf{u}, h}^{n}\right\|_{L^{2}}+\left\|\mathbf{e}_{\mathbf{u}, h}^{n}\right\|_{L^{2}}\right) \\
& \leq c h\left(\tau^{\frac{1}{2}}+h\right)\left(\left\|\nabla \mathbf{e}_{\mathbf{u}, h}^{n}\right\|_{L^{2}}^{2}+\left\|\mathbf{e}_{\mathbf{u}, h}^{n}\right\|_{L^{2}}^{2}\right)+c h^{2}\left(\tau+h^{2}\right)+c\left\|\mathbf{e}_{\mathbf{u}, h}^{n}\right\|_{L^{2}}^{2} .
\end{aligned}
$$

Substituting (5.67)-(5.75) into (5.66) yields

$$
\begin{aligned}
\left|\left(D_{\tau} e_{\rho, h}^{n}, P_{h}\left(\mathbf{u}_{h}^{n} \cdot \mathbf{e}_{\mathbf{u}, h}^{n}\right)\right)\right| \leq & \left(\epsilon+c h \tau^{\frac{1}{2}}+c h^{2}\right)\left(\left\|\nabla \mathbf{e}_{\mathbf{u}, h}^{n}\right\|_{L^{2}}^{2}+\left\|\nabla \mathbf{e}_{\mathbf{u}, h}^{n-1}\right\|_{L^{2}}^{2}\right)+c \epsilon^{-1} h^{2}\left(\tau+h^{2}\right) \\
& +\left(c h^{-1}\left\|\nabla \mathbf{e}_{\mathbf{u}, h}^{n-1}\right\|_{L^{2}}+c \epsilon^{-1}\right)\left(\left\|\mathbf{e}_{\mathbf{u}, h}^{n}\right\|_{L^{2}}^{2}+\left\|e_{\rho, h}^{n}\right\|_{L^{2}}^{2}+\left\|\mathbf{e}_{\mathbf{u}, h}^{n-1}\right\|_{L^{2}}^{2}+\left\|e_{\rho, h}^{n-1}\right\|_{L^{2}}^{2}\right),
\end{aligned}
$$

and therefore

$$
\begin{aligned}
\left|I_{4}^{*}\left(\mathbf{e}_{\mathbf{u}, h}^{n}\right)\right| \leq & \left|\left(D_{\tau}\left(\rho_{\tau}^{n}-P_{h} \rho_{\tau}^{n}\right) \mathbf{u}_{h}^{n}, \mathbf{e}_{\mathbf{u}, h}^{n}\right)\right|+\left|\left(D_{\tau} e_{\rho, h}^{n}, P_{h}\left(\mathbf{u}_{h}^{n} \cdot \mathbf{e}_{\mathbf{u}, h}^{n}\right)\right)\right| \\
\leq & \left\|D_{\tau} \rho_{\tau}^{n}-P_{h} D_{\tau} \rho_{\tau}^{n}\right\|_{L^{\infty}}\left\|\mathbf{u}_{h}^{n}\right\|_{L^{2}}\left\|\mathbf{e}_{\mathbf{u}, h}^{n}\right\|_{L^{2}}+\left|\left(D_{\tau} e_{\rho, h}^{n}, P_{h}\left(\mathbf{u}_{h}^{n} \cdot \mathbf{e}_{\mathbf{u}, h}^{n}\right)\right)\right| \\
\leq & \left(\epsilon+c h \tau^{\frac{1}{2}}+c h^{2}\right)\left(\left\|\nabla \mathbf{e}_{\mathbf{u}, h}^{n}\right\|_{L^{2}}^{2}+\left\|\nabla \mathbf{e}_{\mathbf{u}, h}^{n-1}\right\|_{L^{2}}^{2}\right)+c \epsilon^{-1} h^{2}\left(\tau+h^{2}\right) \\
& +\left(c h^{-1}\left\|\nabla \mathbf{e}_{\mathbf{u}, h}^{n-1}\right\|_{L^{2}}+c \epsilon^{-1}\right)\left(\left\|\mathbf{e}_{\mathbf{u}, h}^{n}\right\|_{L^{2}}^{2}+\left\|e_{\rho, h}^{n}\right\|_{L^{2}}^{2}+\left\|\mathbf{e}_{\mathbf{u}, h}^{n-1}\right\|_{L^{2}}^{2}+\left\|e_{\rho, h}^{n-1}\right\|_{L^{2}}^{2}\right),
\end{aligned}
$$

where $\left\|\mathbf{u}_{h}^{n}\right\|_{L^{2}}$ is bounded as explained in Remark 2.1.

Then substituting (5.58)-(5.65) and (5.76) into (5.56) yields

$$
\begin{aligned}
& \frac{1}{2} D_{\tau}\left\|\sqrt{\rho_{h}^{n}} \mathbf{e}_{\mathbf{u}, h}^{n}\right\|_{L^{2}}^{2}+\left\|\nabla \mathbf{e}_{\mathbf{u}, h}^{n}\right\|_{L^{2}}^{2} \\
& \quad \leq\left|\left(\rho_{h}^{n-1} D_{\tau} \mathbf{e}_{\mathbf{u}, h}^{n}, \mathbf{e}_{\mathbf{u}, h}^{n}\right)\right|+\left|\frac{1}{2}\left(D_{\tau} \rho_{h}^{n} \mathbf{e}_{\mathbf{u}, h}^{n}, \mathbf{e}_{\mathbf{u}, h}^{n}\right)\right|+\left|B\left(\left(\mathbf{e}_{\mathbf{u}, h}^{n}, e_{p, h}^{n}\right),\left(\mathbf{e}_{\mathbf{u}, h}^{n}, e_{p, h}^{n}\right)\right)\right|
\end{aligned}
$$




$$
\begin{aligned}
\leq & \left(\epsilon+c h \tau^{\frac{1}{2}}+c h^{2}+\left(\tau+h^{2}\right)^{\frac{1}{4}}\right)\left(\left\|\nabla \mathbf{e}_{\mathbf{u}, h}^{n}\right\|_{L^{2}}^{2}+\left\|\nabla \mathbf{e}_{\mathbf{u}, h}^{n-1}\right\|_{L^{2}}^{2}\right) \\
& +c \epsilon^{-1} h^{2}\left(\tau+h^{2}\right)+c \epsilon^{-1}\left(\left\|D_{\tau} \mathbf{u}_{\tau}^{n}\right\|_{H^{2}}^{2}+\left\|D_{\tau} p_{\tau}^{n}\right\|_{H^{1}}^{2}\right) h^{4} \\
& +\left(c h^{-1}\left\|\nabla \mathbf{e}_{\mathbf{u}, h}^{n-1}\right\|_{L^{2}}+c \epsilon^{-1}\right)\left(\left\|\mathbf{e}_{\mathbf{u}, h}^{n}\right\|_{L^{2}}^{2}+\left\|e_{\rho, h}^{n}\right\|_{L^{2}}^{2}+\left\|\mathbf{e}_{\mathbf{u}, h}^{n-1}\right\|_{L^{2}}^{2}+\left\|e_{\rho, h}^{n-1}\right\|_{L^{2}}^{2}\right) .
\end{aligned}
$$

By summing up the last inequality times $\tau$ for $n=1, \ldots, k$, and choosing sufficiently small $\epsilon, h$ and $\tau$, we have (the first term on the right-hand side of (5.77) is absorbed by the left-hand side, except a starting term involving $\left.\tau\left\|\nabla \mathbf{e}_{\mathbf{u}, h}^{0}\right\|_{L^{2}}^{2}\right)$

$$
\begin{aligned}
\frac{1}{2}\left\|\sqrt{\rho_{h}^{k}} \mathbf{e}_{\mathbf{u}, h}^{k}\right\|_{L^{2}}^{2} & +\sum_{n=0}^{k} \tau\left\|\nabla \mathbf{e}_{\mathbf{u}, h}^{n}\right\|_{L^{2}}^{2} \leq \frac{1}{2}\left\|\sqrt{\rho_{h}^{0}} \mathbf{e}_{\mathbf{u}, h}^{0}\right\|_{L^{2}}^{2}+c \tau\left\|\nabla \mathbf{e}_{\mathbf{u}, h}^{0}\right\|_{L^{2}}^{2}+c h^{2}\left(\tau+h^{2}\right) \\
& +\tau \sum_{n=1}^{k}\left(c h^{-1}\left\|\nabla \mathbf{e}_{\mathbf{u}, h}^{n-1}\right\|_{L^{2}}+c\right)\left(\left\|\mathbf{e}_{\mathbf{u}, h}^{n}\right\|_{L^{2}}^{2}+\left\|e_{\rho, h}^{n}\right\|_{L^{2}}^{2}+\left\|\mathbf{e}_{\mathbf{u}, h}^{n-1}\right\|_{L^{2}}^{2}+\left\|e_{\rho, h}^{n-1}\right\|_{L^{2}}^{2}\right)
\end{aligned}
$$

for $k=1, \ldots, m$, where we have used (3.10) to estimate the third term on the right-hand side of (5.77), i.e.,

$$
\tau \sum_{n=1}^{k} c\left(\left\|D_{\tau} \mathbf{u}_{\tau}^{n}\right\|_{H^{2}}^{2}+\left\|D_{\tau} p_{\tau}^{n}\right\|_{H^{1}}^{2}\right) h^{4} \leq c h^{4} .
$$

Since $\mathbf{e}_{\mathbf{u}, h}^{0}=\mathbf{R}_{h}\left(\mathbf{u}^{0}, p^{0}\right)-\Pi_{h} \mathbf{u}^{0}$, the estimates (5.6), (5.7) and (5.14) imply

$$
\begin{aligned}
\frac{1}{2}\left\|\sqrt{\rho_{h}^{0}} \mathbf{e}_{\mathbf{u}, h}^{0}\right\|_{L^{2}}^{2} & \leq c h^{4}\left(\left\|u^{0}\right\|_{H^{2}}^{2}+\left\|p^{0}\right\|_{H^{1}}^{2}\right), \\
\tau\left\|\nabla \mathbf{e}_{\mathbf{u}, h}^{0}\right\|_{L^{2}}^{2} & \leq c \tau h^{2}\left(\left\|u^{0}\right\|_{H^{2}}^{2}+\left\|p^{0}\right\|_{H^{1}}^{2}\right) .
\end{aligned}
$$

Substituting the last two inequalities into (5.78) and considering $\epsilon(5.42)+(5.78)$, we have

$$
\begin{aligned}
\epsilon\left\|e_{\rho, h}^{k}\right\|_{L^{2}}^{2} & +\frac{1}{2}\left\|\sqrt{\rho_{h}^{k}} \mathbf{e}_{\mathbf{u}, h}^{k}\right\|_{L^{2}}^{2}+\frac{1}{2} \sum_{n=0}^{k} \tau\left\|\nabla \mathbf{e}_{\mathbf{u}, h}^{n}\right\|_{L^{2}}^{2} \leq \epsilon \sum_{n=1}^{k} \tau\left\|\nabla \mathbf{e}_{\mathbf{u}, h}^{n-1}\right\|_{L^{2}}^{2}+c h^{2}\left(\tau+h^{2}\right) \\
& +\tau \sum_{n=1}^{k}\left(c h^{-1}\left\|\nabla \mathbf{e}_{\mathbf{u}, h}^{n-1}\right\|_{L^{2}}+c\right)\left(\left\|\mathbf{e}_{\mathbf{u}, h}^{n}\right\|_{L^{2}}^{2}+\left\|e_{\rho, h}^{n}\right\|_{L^{2}}^{2}+\left\|\mathbf{e}_{\mathbf{u}, h}^{n-1}\right\|_{L^{2}}^{2}+\left\|e_{\rho, h}^{n-1}\right\|_{L^{2}}^{2}\right), \quad k=1, \ldots, m .
\end{aligned}
$$

Again, by choosing a sufficiently small parameter $\epsilon$ and using (5.46), the first term on the right-hand side above can be absorbed by the left-hand side. Then we obtain

$$
\begin{aligned}
\left\|e_{\rho, h}^{k}\right\|_{L^{2}}^{2} & +\left\|\mathbf{e}_{\mathbf{u}, h}^{k}\right\|_{L^{2}}^{2}+\sum_{n=0}^{k} \tau\left\|\nabla \mathbf{e}_{\mathbf{u}, h}^{n}\right\|_{L^{2}}^{2} \leq c h^{2}\left(\tau+h^{2}\right) \\
& +\tau \sum_{n=1}^{k}\left(c h^{-1}\left\|\nabla \mathbf{e}_{\mathbf{u}, h}^{n-1}\right\|_{L^{2}}+c\right)\left(\left\|\mathbf{e}_{\mathbf{u}, h}^{n}\right\|_{L^{2}}^{2}+\left\|e_{\rho, h}^{n}\right\|_{L^{2}}^{2}+\left\|\mathbf{e}_{\mathbf{u}, h}^{n-1}\right\|_{L^{2}}^{2}+\left\|e_{\rho, h}^{n-1}\right\|_{L^{2}}^{2}\right)
\end{aligned}
$$

for $k=1, \ldots, m$. The fourth inequality of (5.29) implies $\tau \sum_{n=1}^{m}\left(c h^{-1}\left\|\nabla \mathbf{e}_{\mathbf{u}, h}^{n-1}\right\|_{L^{2}}+c\right) \leq c$. Substituting this inequality into (5.79) and applying Gronwall's inequality, we obtain

$$
\max _{1 \leq n \leq m}\left(\left\|e_{\rho, h}^{n}\right\|_{L^{2}}^{2}+\left\|\mathbf{e}_{\mathbf{u}, h}^{n}\right\|_{L^{2}}^{2}\right)+\sum_{n=0}^{m} \tau\left\|\nabla \mathbf{e}_{\mathbf{u}, h}^{n}\right\|_{L^{2}}^{2} \leq c h^{2}\left(\tau+h^{2}\right) .
$$

For sufficiently small mesh size $h$ and $\tau$, the inequality above implies 


$$
\begin{aligned}
\left\|\mathbf{e}_{\mathbf{u}, h}^{m}\right\|_{L^{2}} & \leq h\left(\tau+h^{2}\right)^{\frac{1}{4}}, \\
\left\|\mathbf{e}_{\mathbf{u}, h}^{m}\right\|_{L^{\infty}} & \leq c h^{-1}\left\|\mathbf{e}_{\mathbf{u}, h}^{m}\right\|_{L^{2}} \leq c \sqrt{\tau+h^{2}} \leq 1, \\
\sum_{n=0}^{m} \tau\left\|\nabla \mathbf{e}_{\mathbf{u}, h}^{n}\right\|_{L^{2}}^{2} & \leq c h^{2}\left(\tau+h^{2}\right) \leq h^{2} \sqrt{\tau+h^{2}} .
\end{aligned}
$$

This, together with the induction assumption (5.29), proves the second, third and fourth inequalities of (5.30). The first inequality of (5.30) has already been proved in (5.45). The mathematical induction is closed. Consequently, the estimates (5.30) and (5.80) hold for $m=N$ (with the same constants). These estimates imply

$$
\max _{0 \leq n \leq N}\left(\left\|\rho_{h}^{n}\right\|_{L^{\infty}}+\left\|\mathbf{u}_{h}^{n}\right\|_{L^{\infty}}\right) \leq c
$$

and

$$
\max _{1 \leq n \leq N}\left(\left\|e_{\rho, h}^{n}\right\|_{L^{2}}^{2}+\left\|\mathbf{e}_{\mathbf{u}, h}^{n}\right\|_{L^{2}}^{2}\right)+\sum_{n=0}^{N} \tau\left\|\nabla \mathbf{e}_{\mathbf{u}, h}^{n}\right\|_{L^{2}}^{2} \leq c h^{2}\left(\tau+h^{2}\right) .
$$

This proves (5.22) in view of the first inequality of (5.30) and the derivation of (5.32).

\subsection{Improving the estimate of $D_{\tau} e_{\rho, h}^{n}$}

By using (5.82), we re-estimate $\left\|D_{\tau} e_{\rho, h}^{n}\right\|_{L^{2}}$ by taking $\varphi_{h}=D_{\tau} e_{\rho, h}^{n}$ in (5.34). Since

$$
\left(D_{\tau}\left(\rho_{\tau}^{n}-P_{h} \rho_{\tau}^{n}\right), D_{\tau} e_{\rho, h}^{n}\right)=\left(D_{\tau} \rho_{\tau}^{n}-P_{h} D_{\tau} \rho_{\tau}^{n}, D_{\tau} e_{\rho, h}^{n}\right)=0
$$

we obtain

$$
\begin{aligned}
\left\|D_{\tau} e_{\rho, h}^{n}\right\|_{L^{2}} \leq & \left\|\mathbf{u}_{h}^{n-1} \cdot \nabla\left(\rho_{\tau}^{n}-P_{h} \rho_{\tau}^{n}\right)\right\|_{L^{2}}+\frac{1}{2}\left\|\nabla \cdot \mathbf{u}_{h}^{n-1}\left(\rho_{\tau}^{n}-P_{h} \rho_{\tau}^{n}\right)\right\|_{L^{2}} \\
& +\left\|\mathbf{u}_{h}^{n-1} \cdot \nabla e_{\rho, h}^{n}\right\|_{L^{2}}+\frac{1}{2}\left\|\nabla \cdot \mathbf{u}_{h}^{n-1} e_{\rho, h}^{n}\right\|_{L^{2}} \\
& +\left\|\mathbf{e}_{\mathbf{u}, h}^{n-1} \cdot \nabla \rho_{\tau}^{n}\right\|_{L^{2}}+\frac{1}{2}\left\|\nabla \cdot \mathbf{e}_{\mathbf{u}, h}^{n-1} \rho_{\tau}^{n}\right\|_{L^{2}} \\
& +\left\|\left(\mathbf{u}_{\tau}^{n-1}-\mathbf{R}_{h}\left(\mathbf{u}_{\tau}^{n-1}, p_{\tau}^{n-1}\right)\right) \cdot \nabla \rho_{\tau}^{n}\right\|_{L^{2}} \\
& +\frac{1}{2}\left\|\rho_{\tau}^{n} \nabla \cdot\left(\mathbf{u}_{\tau}^{n-1}-\mathbf{R}_{h}\left(\mathbf{u}_{\tau}^{n-1}, p_{\tau}^{n-1}\right)\right)\right\|_{L^{2}} \\
= & : \sum_{k=1}^{5} J_{k}^{*}
\end{aligned}
$$

where

$$
\begin{array}{rlrl}
J_{1}^{*} & \leq\left\|\mathbf{u}_{h}^{n-1}\right\|_{L^{\infty}} c h\left\|\rho_{\tau}^{n}\right\|_{H^{2}}+c\left\|\nabla \cdot \mathbf{u}_{h}^{n-1}\right\|_{L^{\infty}}\left\|\rho_{\tau}^{n}-P_{h} \rho_{\tau}^{n}\right\|_{L^{2}} & & \\
& \leq\left\|\mathbf{u}_{h}^{n-1}\right\|_{L^{\infty}} c h\left\|\rho_{\tau}^{n}\right\|_{H^{2}}+c h^{-1}\left\|\mathbf{u}_{h}^{n-1}\right\|_{L^{\infty}} c h^{2}\left\|\rho_{\tau}^{n}\right\|_{H^{2}} & & \text { (inverse inequality) } \\
& \leq c h, \quad\left(\text { use }(3.9) \text { to estimate }\left\|\rho_{\tau}^{n}\right\|_{H^{2}},\right. \text { and (5.81) } & \text { to estimate } \left.\left\|\mathbf{u}_{h}^{n-1}\right\|_{L^{\infty}}\right) \\
J_{2}^{*} & \leq\left\|\mathbf{u}_{h}^{n-1}\right\|_{L^{\infty}}\left\|\nabla e_{\rho, h}^{n}\right\|_{L^{2}}+\left\|\nabla \cdot \mathbf{u}_{h}^{n-1}\right\|_{L^{\infty}}\left\|e_{\rho, h}^{n}\right\|_{L^{2}} & \\
& \leq\left\|\mathbf{u}_{h}^{n-1}\right\|_{L^{\infty}} c h^{-1}\left\|e_{\rho, h}^{n}\right\|_{L^{2}}+c h^{-1}\left\|\mathbf{u}_{h}^{n-1}\right\|_{L^{\infty}}\left\|e_{\rho, h}^{n}\right\|_{L^{2}} & & \text { (inverse inequality) } \\
& \leq\left\|\mathbf{u}_{h}^{n-1}\right\|_{L^{\infty}} c h^{-1} c h\left(\tau^{\frac{1}{2}}+h\right)+c h^{-1}\left\|\mathbf{u}_{h}^{n-1}\right\|_{L^{\infty}} c h\left(\tau^{\frac{1}{2}}+h\right) & & \text { (use (5.82) to estimate } \left.\left\|e_{\rho}^{n}\right\|_{L^{2}}\right) \\
& \leq c\left(\tau^{\frac{1}{2}}+h\right), & & \text { (use (5.81) to estimate } \left.\left\|\mathbf{u}_{h}^{n-1}\right\|_{L^{\infty}}\right)
\end{array}
$$




$$
\begin{aligned}
& J_{3}^{*} \leq\left\|\mathbf{e}_{\mathbf{u}, h}^{n-1}\right\|_{L^{2}}\left\|\nabla \rho_{\tau}^{n}\right\|_{L^{\infty}}+\left\|\nabla \cdot \mathbf{e}_{\mathbf{u}, h}^{n-1}\right\|_{L^{2}}\left\|\rho_{\tau}^{n}\right\|_{L^{\infty}} \\
& \leq\left\|\mathbf{e}_{\mathbf{u}, h}^{n-1}\right\|_{L^{2}} \mid\left\|\nabla \rho_{\tau}^{n}\right\|_{L^{\infty}}+c h^{-1}\left\|\mathbf{e}_{\mathbf{u}, h}^{n-1}\right\|_{L^{2}}\left\|\rho_{\tau}^{n}\right\|_{L^{\infty}} \quad \text { (inverse inequality) } \\
& \leq \operatorname{ch}\left(\tau^{\frac{1}{2}}+h\right)\left\|\nabla \rho_{\tau}^{n}\right\|_{L^{\infty}}+c h^{-1} \operatorname{ch}\left(\tau^{\frac{1}{2}}+h\right)\left\|\rho_{\tau}^{n}\right\|_{L^{\infty}} \quad \text { (use (5.82) to estimate }\left\|\mathbf{e}_{\mathbf{u}, h}^{n-1}\right\|_{L^{2}} \text { ) } \\
& \left.\leq c\left(\tau^{\frac{1}{2}}+h\right), \quad \text { (use (3.9) to estimate }\left\|\nabla \rho_{\tau}^{n}\right\|_{L^{\infty}} \text { and }\left\|\rho_{\tau}^{n}\right\|_{L^{\infty}}\right) \\
& J_{4}^{*} \leq c h^{2}\left(\left\|\mathbf{u}_{\tau}^{n-1}\right\|_{H^{2}}+\left\|p_{\tau}^{n-1}\right\|_{H^{1}}\right)\left\|\nabla \rho_{\tau}^{n}\right\|_{L^{\infty}} \leq c h^{2}, \quad \text { (use (3.9)) } \\
& J_{5}^{*} \leq c\left\|\rho_{\tau}^{n}\right\|_{L^{\infty}}\left\|\nabla \cdot\left(\mathbf{u}_{\tau}^{n-1}-\mathbf{R}_{h}\left(\mathbf{u}_{\tau}^{n-1}, p_{\tau}^{n-1}\right)\right)\right\|_{L^{2}} \\
& \leq c\left\|\rho_{\tau}^{n}\right\|_{L^{\infty}} h\left(\left\|\mathbf{u}_{\tau}^{n-1}\right\|_{H^{2}}+\left\|p_{\tau}^{n-1}\right\|_{H^{1}}\right) \\
& \left.\leq \text { ch. } \quad \text { (use (3.9) to estimate }\left\|\rho_{\tau}^{n}\right\|_{L^{\infty}},\left\|\mathbf{u}_{\tau}^{n-1}\right\|_{H^{2}} \text { and }\left\|p_{\tau}^{n-1}\right\|_{H^{1}}\right)
\end{aligned}
$$

Substituting (5.84)-(5.88) into (5.83) yields

$$
\max _{1 \leq n \leq N}\left\|D_{\tau} e_{\rho, h}^{n}\right\|_{L^{2}} \leq c\left(\tau^{\frac{1}{2}}+h\right) .
$$

which improves the estimate (5.53) obtained in Section 5.5.

\subsection{Estimates of $e_{p, h}^{n}$}

We estimate $\left\|D_{\tau} \mathbf{e}_{\mathbf{u}, h}^{n}\right\|_{L^{2}}$ by taking $\mathbf{v}_{h}=D_{\tau} \mathbf{e}_{\mathbf{u}, h}^{n}$ and $q_{h}=0$ in (5.56). We obtain

$$
\begin{aligned}
\left(\rho_{h}^{n-1} D_{\tau} \mathbf{e}_{\mathbf{u}, h}^{n}, D_{\tau} \mathbf{e}_{\mathbf{u}, h}^{n}\right)+B\left(\left(\mathbf{e}_{\mathbf{u}, h}^{n}, e_{p, h}^{n}\right),\left(D_{\tau} \mathbf{e}_{\mathbf{u}, h}^{n}, 0\right)\right) & =\left(-\frac{1}{2} D_{\tau} \rho_{h}^{n} \mathbf{e}_{\mathbf{u}, h}^{n}, D_{\tau} \mathbf{e}_{\mathbf{u}, h}^{n}\right)+\sum_{j=1}^{10} I_{j}^{*}\left(D_{\tau} \mathbf{e}_{\mathbf{u}, h}^{n}\right) \\
& =\left(-\frac{1}{2} D_{\tau} \rho_{\tau}^{n} \mathbf{e}_{\mathbf{u}, h}^{n}, D_{\tau} \mathbf{e}_{\mathbf{u}, h}^{n}\right)+\sum_{j=1}^{10}\left(K_{j}, D_{\tau} \mathbf{e}_{\mathbf{u}, h}^{n}\right) .
\end{aligned}
$$

where $\left(K_{j}, \mathbf{v}_{h}\right)=I_{j}^{*}\left(\mathbf{v}_{h}\right)$ for $j \neq 3$ and

$$
\left(K_{3}, \mathbf{v}_{h}\right):=\left(\frac{1}{2} D_{\tau}\left(\rho_{\tau}^{n}-\rho_{h}^{n}\right) \mathbf{e}_{\mathbf{u}, h}^{n}, \mathbf{v}_{h}\right)+I_{3}^{*}\left(\mathbf{v}_{h}\right)=\left(-\frac{1}{2}\left(D_{\tau} \rho_{\tau}^{n}\left(\mathbf{u}_{\tau}^{n}-\mathbf{R}_{h}\left(\mathbf{u}_{\tau}^{n}, p_{\tau}^{n}\right)\right)\right), \mathbf{v}_{h}\right) .
$$

Thus

$$
\begin{aligned}
\left\|\frac{1}{2} D_{\tau} \rho_{\tau}^{n} \mathbf{e}_{\mathbf{u}, h}^{n}\right\|_{L^{2}} & \leq \frac{1}{2}\left\|D_{\tau} \rho_{\tau}^{n}\right\|_{L^{\infty}}\left\|\mathbf{e}_{\mathbf{u}, h}^{n}\right\|_{L^{2}} \\
& \leq \frac{1}{2}\left(\left\|D_{\tau} e_{\rho}^{n}\right\|_{L^{\infty}}+\left\|D_{\tau} \rho^{n}\right\|_{L^{\infty}}\right)\left\|\mathbf{e}_{\mathbf{u}, h}^{n}\right\|_{L^{2}} \\
& \leq c\left(\left\|D_{\tau} e_{\rho}^{n}\right\|_{H^{2}}+\left\|D_{\tau} \rho^{n}\right\|_{H^{2}}\right)\left\|\mathbf{e}_{\mathbf{u}, h}^{n}\right\|_{L^{2}} \\
& \leq \operatorname{ch}\left(\tau^{\frac{1}{2}}+h\right), \quad(\text { use }(3.7) \text { and }(5.82)) \\
\left\|K_{1}\right\|_{L^{2}} & =\left\|\rho_{h}^{n-1} D_{\tau}\left(\mathbf{u}_{\tau}^{n}-\mathbf{R}_{h}\left(\mathbf{u}_{\tau}^{n}, p_{\tau}^{n}\right)\right)\right\|_{L^{2}} \\
& \leq\left\|\rho_{h}^{n-1}\right\|_{L^{\infty}}\left\|D_{\tau}\left(\mathbf{u}_{\tau}^{n}-\mathbf{R}_{h}\left(\mathbf{u}_{\tau}^{n}, p_{\tau}^{n}\right)\right)\right\|_{L^{2}} \\
& =\left\|\rho_{h}^{n-1}\right\|_{L^{\infty}}\left\|D_{\tau} \mathbf{u}_{\tau}^{n}-\mathbf{R}_{h}\left(D_{\tau} \mathbf{u}_{\tau}^{n}, D_{\tau} p_{\tau}^{n}\right)\right\|_{L^{2}} \\
& \leq\left\|\rho_{h}^{n-1}\right\|_{L^{\infty}} c h^{2}\left(\left\|D_{\tau} \mathbf{u}_{\tau}^{n}\right\|_{H^{2}}+\left\|D_{\tau} p_{\tau}^{n}\right\|_{H^{1}}\right) \\
& \leq c h^{2}\left(\left\|D_{\tau} \mathbf{u}_{\tau}^{n}\right\|_{H^{2}}+\left\|D_{\tau} p_{\tau}^{n}\right\|_{H^{1}}\right), \quad\left(\text { use }(5.46) \text { to control }\left\|\rho_{h}^{n-1}\right\|_{L^{\infty}}\right) \\
\left\|K_{2}\right\|_{L^{2}} & =\left\|\left(\rho_{\tau}^{n-1}-P_{h} \rho_{\tau}^{n-1}\right) D_{\tau} \mathbf{u}_{\tau}^{n}+e_{\rho, h}^{n-1} D_{\tau} \mathbf{u}_{\tau}^{n}\right\|_{L^{2}} \\
& \leq\left(\left\|\rho_{\tau}^{n-1}-P_{h} \rho_{\tau}^{n-1}\right\|_{L^{2}}+\left\|e_{\rho, h}^{n-1}\right\|_{L^{2}}\right)\left\|D_{\tau} \mathbf{u}_{\tau}^{n}\right\|_{L^{\infty}}
\end{aligned}
$$




$$
\begin{aligned}
& \leq\left(\operatorname{ch}^{2}\left\|\rho_{\tau}^{n-1}\right\|_{H^{2}}+\left\|e_{\rho, h}^{n-1}\right\|_{L^{2}}\right)\left\|D_{\tau} \mathbf{u}_{\tau}^{n}\right\|_{L^{\infty}} \\
& \leq c\left(h^{2}+\left\|e_{\rho, h}^{n-1}\right\|_{L^{2}}\right) \quad\left(\text { use (3.9) to control }\left\|\rho_{\tau}^{n-1}\right\|_{H^{2}} \text { and }\left\|D_{\tau} \mathbf{u}_{\tau}^{n}\right\|_{L^{\infty}}\right) \\
& \left.\leq \operatorname{ch}\left(\tau^{\frac{1}{2}}+h\right), \quad \quad \text { (use }(5.82) \text { to estimate }\left\|e_{\rho, h}^{n-1}\right\|_{L^{2}}\right) \\
& \left\|K_{3}\right\|_{L^{2}}=\left\|\frac{1}{2} D_{\tau} \rho_{\tau}^{n}\left(\mathbf{u}_{\tau}^{n}-\mathbf{R}_{h}\left(\mathbf{u}_{\tau}^{n}, p_{\tau}^{n}\right)\right)\right\|_{L^{2}} \\
& \leq c\left\|D_{\tau} \rho_{\tau}^{n}\right\|_{L^{\infty}}\left\|\mathbf{u}_{\tau}^{n}-\mathbf{R}_{h}\left(\mathbf{u}_{\tau}^{n}, p_{\tau}^{n}\right)\right\|_{L^{2}} \\
& \leq c\left(\left\|D_{\tau} e_{\rho}^{n}\right\|_{L^{\infty}}+\left\|D_{\tau} \rho^{n}\right\|_{L^{\infty}}\right)\left\|\mathbf{u}_{\tau}^{n}-\mathbf{R}_{h}\left(\mathbf{u}_{\tau}^{n}, p_{\tau}^{n}\right)\right\|_{L^{2}} \\
& \leq c\left(\left\|D_{\tau} e_{\rho}^{n}\right\|_{H^{2}}+\left\|D_{\tau} \rho^{n}\right\|_{H^{2}}\right) h^{2}\left(\left\|\mathbf{u}_{\tau}^{n}\right\|_{H^{2}}+\left\|p_{\tau}^{n}\right\|_{H^{1}}\right) \\
& \leq c h^{2}, \quad \text { (use (3.7) to control }\left\|D_{\tau} e_{\rho}^{n}\right\|_{H^{2}} \text { ) } \\
& \text { (use (3.9) to control }\left\|\mathbf{u}_{\tau}^{n}\right\|_{H^{2}}+\left\|p_{\tau}^{n}\right\|_{H^{1}} \text { ) } \\
& \left.\left\|K_{4}\right\|_{L^{2}}=\| D_{\tau} \rho_{\tau}^{n}-P_{h} D_{\tau} \rho_{\tau}^{n}\right) \mathbf{u}_{h}^{n}+D_{\tau} e_{\rho, h}^{n} \mathbf{u}_{h}^{n} \|_{L^{2}} \\
& \leq\left(\left\|D_{\tau} \rho_{\tau}^{n}-P_{h} D_{\tau} \rho_{\tau}^{n}\right\|_{L^{2}}+\left\|D_{\tau} e_{\rho, h}^{n}\right\|_{L^{2}}\right)\left\|\mathbf{u}_{h}^{n}\right\|_{L^{\infty}} \\
& \leq\left(\operatorname{ch}^{2}\left\|D_{\tau} \rho_{\tau}^{n}\right\|_{H^{2}}+\left\|D_{\tau} e_{\rho, h}^{n}\right\|_{L^{2}}\right)\left\|\mathbf{u}_{h}^{n}\right\|_{L^{\infty}} \\
& \leq c h^{2}+c\left\|D_{\tau} e_{\rho, h}^{n}\right\|_{L^{2}} \quad \text { (use (3.7) to estimate }\left\|D_{\tau} \rho_{\tau}^{n}\right\|_{H^{2}} \text { ) } \\
& \text { (use (5.81) to estimate }\left\|\mathbf{u}_{h}^{n}\right\|_{L^{\infty}} \text { ) } \\
& \leq c\left(\tau^{\frac{1}{2}}+h\right), \quad \quad(\text { use }(5.89)) \\
& \left\|K_{5}\right\|_{L^{2}}=\left\|\rho_{h}^{n} \mathbf{u}_{h}^{n-1} \nabla \mathbf{e}_{\mathbf{u}, h}^{n}+\rho_{h}^{n} \mathbf{u}_{h}^{n-1} \nabla\left(\mathbf{u}_{\tau}^{n}-\mathbf{R}_{h}\left(\mathbf{u}_{\tau}^{n}, p_{\tau}^{n}\right)\right)\right\|_{L^{2}} \\
& \leq\left\|\rho_{h}^{n}\right\|_{L^{\infty}}\left\|\mathbf{u}_{h}^{n-1}\right\|_{L^{\infty}}\left(\left\|\nabla \mathbf{e}_{\mathbf{u}, h}^{n}\right\|_{L^{2}}+\left\|\nabla\left(\mathbf{u}_{\tau}^{n}-\mathbf{R}_{h}\left(\mathbf{u}_{\tau}^{n}, p_{\tau}^{n}\right)\right)\right\|_{L^{2}}\right) \\
& \leq\left\|\rho_{h}^{n}\right\|_{L^{\infty}}\left\|\mathbf{u}_{h}^{n-1}\right\|_{L^{\infty}}\left(\left\|\nabla \mathbf{e}_{\mathbf{u}, h}^{n}\right\|_{L^{2}}+c h \tau^{\frac{1}{2}}+c h^{2}\right) \quad \text { (use (5.19)) } \\
& \leq\left\|\rho_{h}^{n}\right\|_{L^{\infty}}\left\|\mathbf{u}_{h}^{n-1}\right\|_{L^{\infty}}\left(c h^{-1}\left\|\mathbf{e}_{\mathbf{u}, h}^{n}\right\|_{L^{2}}+c h \tau^{\frac{1}{2}}+c h^{2}\right) \quad \text { (use inverse inequality) } \\
& \leq c\left(\tau^{\frac{1}{2}}+h\right), \quad \text { (use (5.82) for }\left\|\mathbf{e}_{\mathbf{u}, h}^{n}\right\|_{L^{2}} \text {, and (5.81) for }\left\|\rho_{h}^{n}\right\|_{L^{\infty}} \text { and }\left\|\mathbf{u}_{h}^{n-1}\right\|_{L^{\infty}} \text { ) } \\
& \left\|K_{6}\right\|_{L^{2}}=\left\|\rho_{h}^{n}\left(\mathbf{u}_{\tau}^{n-1}-\mathbf{R}_{h}\left(\mathbf{u}_{\tau}^{n-1}, p_{\tau}^{n-1}\right)\right) \cdot \nabla \mathbf{u}_{\tau}^{n}+\rho_{h}^{n} \mathbf{e}_{\mathbf{u}, h}^{n-1} \cdot \nabla \mathbf{u}_{\tau}^{n}\right\|_{L^{2}} \\
& \leq\left\|\rho_{h}^{n}\right\|_{L^{\infty}}\left(\left\|\mathbf{u}_{\tau}^{n-1}-\mathbf{R}_{h}\left(\mathbf{u}_{\tau}^{n-1}, p_{\tau}^{n-1}\right)\right\|_{L^{2}}+\left\|\mathbf{e}_{\mathbf{u}, h}^{n-1}\right\|_{L^{2}}\right)\left\|\nabla \mathbf{u}_{\tau}^{n}\right\|_{L^{\infty}} \\
& \leq\left\|\rho_{h}^{n}\right\|_{L^{\infty}}\left(\operatorname{ch}^{2}\left(\left\|\mathbf{u}_{\tau}^{n-1}\right\|_{H^{2}}+\left\|p_{\tau}^{n-1}\right\|_{H^{1}}\right)+\left\|\mathbf{e}_{\mathbf{u}, h}^{n-1}\right\|_{L^{2}}\right)\left\|\nabla \mathbf{u}_{\tau}^{n}\right\|_{L^{\infty}} \\
& \leq c\left(h^{2}+\left\|\mathbf{e}_{\mathbf{u}, h}^{n-1}\right\|_{L^{2}}\right) \quad\left(\text { use (5.81) to estimate }\left\|\rho_{h}^{n}\right\|_{L^{\infty}}\right) \\
& \text { (use (3.9) to estimate }\left\|\nabla \mathbf{u}_{\tau}^{n}\right\|_{L^{\infty}} \text { ) } \\
& \leq \operatorname{ch}\left(\tau^{\frac{1}{2}}+h\right), \quad \quad(\text { use }(5.82)) \\
& \left\|K_{7}\right\|_{L^{2}}=\left\|\left(\rho_{\tau}^{n}-P_{h} \rho_{\tau}^{n}\right) \mathbf{u}_{\tau}^{n-1} \cdot \nabla \mathbf{u}_{\tau}^{n}+e_{\rho, h}^{n} \mathbf{u}_{\tau}^{n-1} \cdot \nabla \mathbf{u}_{\tau}^{n}\right\|_{L^{2}} \\
& \leq\left(\left\|\rho_{\tau}^{n}-P_{h} \rho_{\tau}^{n}\right\|_{L^{2}}+\left\|e_{\rho, h}^{n}\right\|_{L^{2}}\right)\left\|\mathbf{u}_{\tau}^{n-1}\right\|_{L^{\infty}}\left\|\nabla \mathbf{u}_{\tau}^{n}\right\|_{L^{\infty}} \\
& \leq\left(c h^{2}\left\|\rho_{\tau}^{n}\right\|_{H^{2}}+\left\|e_{\rho, h}^{n}\right\|_{L^{2}}\right)\left\|\mathbf{u}_{\tau}^{n-1}\right\|_{L^{\infty}}\left\|\nabla \mathbf{u}_{\tau}^{n}\right\|_{L^{\infty}} \\
& \left.\leq c\left(h^{2}+\left\|e_{\rho, h}^{n}\right\|_{L^{2}}\right) \quad \text { (use (3.9) to estimate }\left\|\rho_{\tau}^{n}\right\|_{H^{2}},\left\|\mathbf{u}_{\tau}^{n-1}\right\|_{L^{\infty}} \text { and }\left\|\nabla \mathbf{u}_{\tau}^{n}\right\|_{L^{\infty}}\right) \\
& \leq \operatorname{ch}\left(\tau^{\frac{1}{2}}+h\right), \quad(\text { use }(5.82))
\end{aligned}
$$




$$
\begin{aligned}
& \left\|K_{8}\right\|_{L^{2}}=\frac{1}{2}\left\|\nabla \cdot\left(\rho_{h}^{n} \mathbf{u}_{h}^{n-1} \otimes \mathbf{e}_{\mathbf{u}, h}^{n}\right)+\nabla \cdot\left(\rho_{h}^{n} \mathbf{u}_{h}^{n-1} \otimes\left(\mathbf{u}_{\tau}^{n}-\mathbf{R}_{h}\left(\mathbf{u}_{\tau}^{n}, p_{\tau}^{n}\right)\right)\right)\right\|_{L^{2}} \\
& \leq\left\|\rho_{h}^{n} \mathbf{u}_{h}^{n-1}\right\|_{L^{\infty}}\left(\left\|\nabla \mathbf{e}_{\mathbf{u}, h}^{n}\right\|_{L^{2}}+\left\|\nabla\left(\mathbf{u}_{\tau}^{n}-\mathbf{R}_{h}\left(\mathbf{u}_{\tau}^{n}, p_{\tau}^{n}\right)\right)\right\|_{L^{2}}\right) \\
& +\left\|\nabla\left(\rho_{h}^{n} \mathbf{u}_{h}^{n-1}\right)\right\|_{L^{\infty}}\left(\left\|\mathbf{e}_{\mathbf{u}, h}^{n}\right\|_{L^{2}}+\left\|\mathbf{u}_{\tau}^{n}-\mathbf{R}_{h}\left(\mathbf{u}_{\tau}^{n}, p_{\tau}^{n}\right)\right\|_{L^{2}}\right) \\
& \leq\left\|\rho_{h}^{n} \mathbf{u}_{h}^{n-1}\right\|_{L^{\infty}}\left(c h^{-1}\left\|\mathbf{e}_{\mathbf{u}, h}^{n}\right\|_{L^{2}}+\operatorname{ch}\left(\tau^{\frac{1}{2}}+h\right)\right) \\
& \text { (use inverse inequality and (5.19)) } \\
& \left\|\nabla \cdot\left(\rho_{h}^{n} \mathbf{u}_{h}^{n-1}\right)\right\|_{L^{\infty}}\left(\operatorname{ch}\left(\tau^{\frac{1}{2}}+h\right)+c h^{2}\left(\tau^{\frac{1}{2}}+h\right)\right) \quad \text { (use (5.80) and (5.18)) } \\
& \leq\left\|\rho_{h}^{n} \mathbf{u}_{h}^{n-1}\right\|_{L^{\infty}}\left(\tau^{\frac{1}{2}}+h+\operatorname{ch}\left(\tau^{\frac{1}{2}}+h\right)\right) \\
& \text { (use (5.80)) } \\
& +\left\|\rho_{h}^{n} \mathbf{u}_{h}^{n-1}\right\|_{L^{\infty}}\left(c\left(\tau^{\frac{1}{2}}+h\right)+\operatorname{ch}\left(\tau^{\frac{1}{2}}+h\right)\right) \quad \text { (inverse inequality) } \\
& \leq c\left(\tau^{\frac{1}{2}}+h\right), \quad\left(\text { use }(5.81) \text { to estimate }\left\|\rho_{h}^{n}\right\|_{L^{\infty}} \text { and }\left\|\mathbf{u}_{h}^{n-1}\right\|_{L^{\infty}}\right) \\
& \left\|K_{9}\right\|_{L^{2}}=\frac{1}{2}\left\|\nabla \cdot\left(\rho_{h}^{n}\left(\mathbf{u}_{\tau}^{n-1}-\mathbf{R}_{h}\left(\mathbf{u}_{\tau}^{n-1}, p_{\tau}^{n-1}\right)\right) \otimes \mathbf{u}_{\tau}^{n}\right)+\nabla \cdot\left(\rho_{h}^{n} \mathbf{e}_{\mathbf{u}, h}^{n-1} \otimes \mathbf{u}_{\tau}^{n}\right)\right\|_{L^{2}} \\
& \leq\left\|\nabla\left(\rho_{h}^{n}\left(\mathbf{u}_{\tau}^{n-1}-\mathbf{R}_{h}\left(\mathbf{u}_{\tau}^{n-1}, p_{\tau}^{n-1}\right)\right)\right)\right\|_{L^{2}}\left\|\mathbf{u}_{\tau}^{n}\right\|_{L^{\infty}}+\left\|\nabla\left(\rho_{h}^{n} \mathbf{e}_{\mathbf{u}, h}^{n-1}\right)\right\|_{L^{2}}\left\|\mathbf{u}_{\tau}^{n}\right\|_{L^{\infty}} \\
& +\left\|\rho_{h}^{n}\left(\mathbf{u}_{\tau}^{n-1}-\mathbf{R}_{h}\left(\mathbf{u}_{\tau}^{n-1}, p_{\tau}^{n-1}\right)\right)\right\|_{L^{2}}\left\|\nabla \mathbf{u}_{\tau}^{n}\right\|_{L^{\infty}}+\left\|\rho_{h}^{n} \mathbf{e}_{\mathbf{u}, h}^{n-1}\right\|_{L^{2}}\left\|\nabla \mathbf{u}_{\tau}^{n}\right\|_{L^{\infty}} \\
& \leq\left\|\rho_{h}^{n}\right\|_{L^{\infty}}\left\|\nabla\left(\mathbf{u}_{\tau}^{n-1}-\mathbf{R}_{h}\left(\mathbf{u}_{\tau}^{n-1}, p_{\tau}^{n-1}\right)\right)\right\|_{L^{2}}\left\|\mathbf{u}_{\tau}^{n}\right\|_{L^{\infty}} \\
& +\left\|\nabla \rho_{h}^{n}\right\|_{L^{\infty}}\left\|\mathbf{u}_{\tau}^{n-1}-\mathbf{R}_{h}\left(\mathbf{u}_{\tau}^{n-1}, p_{\tau}^{n-1}\right)\right\|_{L^{2}}\left\|\mathbf{u}_{\tau}^{n}\right\|_{L^{\infty}} \\
& +\left\|\rho_{h}^{n}\right\|_{L^{\infty}}\left\|\nabla \mathbf{e}_{\mathbf{u}, h}^{n-1}\right\|_{L^{2}}\left\|\mathbf{u}_{\tau}^{n}\right\|_{L^{\infty}}+\left\|\nabla \rho_{h}^{n}\right\|_{L^{\infty}}\left\|\mathbf{e}_{\mathbf{u}, h}^{n-1}\right\|_{L^{2}}\left\|\mathbf{u}_{\tau}^{n}\right\|_{L^{\infty}} \\
& +\left\|\rho_{h}^{n}\left(\mathbf{u}_{\tau}^{n-1}-\mathbf{R}_{h}\left(\mathbf{u}_{\tau}^{n-1}, p_{\tau}^{n-1}\right)\right)\right\|_{L^{2}}\left\|\nabla \mathbf{u}_{\tau}^{n}\right\|_{L^{\infty}}+\left\|\rho_{h}^{n} \mathbf{e}_{\mathbf{u}, h}^{n-1}\right\|_{L^{2}}\left\|\nabla \mathbf{u}_{\tau}^{n}\right\|_{L^{\infty}} \\
& \leq c\left\|\rho_{h}^{n}\right\|_{L^{\infty}}\left(\operatorname{ch} \tau^{\frac{1}{2}}+c h^{2}\right)\left\|\mathbf{u}_{\tau}^{n}\right\|_{L^{\infty}} \\
& +c\left\|\nabla \rho_{h}^{n}\right\|_{L^{\infty}}\left(c h^{2} \tau^{\frac{1}{2}}+c h^{3}\right)\left\|\mathbf{u}_{\tau}^{n}\right\|_{L^{\infty}} \\
& +c\left\|\rho_{h}^{n}\right\|_{L^{\infty}}\left(\tau^{\frac{1}{2}}+h\right)\left\|\mathbf{u}_{\tau}^{n}\right\|_{L^{\infty}}+c\left\|\nabla \rho_{h}^{n}\right\|_{L^{\infty}} h\left(\tau^{\frac{1}{2}}+h\right)\left\|\mathbf{u}_{\tau}^{n}\right\|_{L^{\infty}} \quad \text { (use (5.82)) } \\
& +c\left\|\rho_{h}^{n}\right\|_{L^{\infty}}\left(c h^{2} \tau^{\frac{1}{2}}+c h^{3}\right)\left\|\nabla \mathbf{u}_{\tau}^{n}\right\|_{L^{\infty}}+c\left\|\rho_{h}^{n}\right\|_{L^{\infty}} h\left(\tau^{\frac{1}{2}}+h\right)\left\|\nabla \mathbf{u}_{\tau}^{n}\right\|_{L^{\infty}}
\end{aligned}
$$$$
\text { (use (5.18) and (5.82)) }
$$$$
\left.\leq c\left(\tau^{\frac{1}{2}}+h\right), \quad \quad \text { (use (3.9) to estimate }\left\|\mathbf{u}_{\tau}^{n}\right\|_{L^{\infty}} \text { and }\left\|\nabla \mathbf{u}_{\tau}^{n}\right\|_{L^{\infty}}\right)
$$
(use inverse inequality to estimate $\left\|\nabla \rho_{h}^{n}\right\|_{L^{\infty}}$ ) (use (5.81) to estimate $\left\|\rho_{h}^{n}\right\|_{L^{\infty}}$ ),

$$
\begin{aligned}
\left\|K_{10}\right\|_{L^{2}}= & \left\|\nabla \cdot\left(\left(\rho_{\tau}^{n}-P_{h} \rho_{\tau}^{n}\right) \mathbf{u}_{\tau}^{n-1} \otimes \mathbf{u}_{\tau}^{n}\right)+\nabla \cdot\left(e_{\rho, h}^{n} \mathbf{u}_{\tau}^{n-1} \otimes \mathbf{u}_{\tau}^{n}\right)\right\|_{L^{2}} \\
\leq & \left\|\nabla\left(\rho_{\tau}^{n}-P_{h} \rho_{\tau}^{n}\right)\right\|_{L^{2}}\left\|\mathbf{u}_{\tau}^{n-1}\right\|_{L^{\infty}}\left\|\mathbf{u}_{\tau}^{n}\right\|_{L^{\infty}}+\left\|\nabla e_{\rho, h}^{n}\right\|_{L^{2}}\left\|\mathbf{u}_{\tau}^{n-1}\right\|_{L^{\infty}}\left\|\mathbf{u}_{\tau}^{n}\right\|_{L^{\infty}} \\
& +\left\|\rho_{\tau}^{n}-P_{h} \rho_{\tau}^{n}\right\|_{L^{2}}\left\|\nabla \mathbf{u}_{\tau}^{n-1}\right\|_{L^{\infty}}\left\|\mathbf{u}_{\tau}^{n}\right\|_{L^{\infty}}+\left\|e_{\rho, h}^{n}\right\|_{L^{2}}\left\|\nabla \mathbf{u}_{\tau}^{n-1}\right\|_{L^{\infty}}\left\|\mathbf{u}_{\tau}^{n}\right\|_{L^{\infty}} \\
& +\left\|\rho_{\tau}^{n}-P_{h} \rho_{\tau}^{n}\right\|_{L^{2}}\left\|\mathbf{u}_{\tau}^{n-1}\right\|_{L^{\infty}}\left\|\nabla \mathbf{u}_{\tau}^{n}\right\|_{L^{\infty}}+\left\|e_{\rho, h}^{n}\right\|_{L^{2}}\left\|\mathbf{u}_{\tau}^{n-1}\right\|_{L^{\infty}}\left\|\nabla \mathbf{u}_{\tau}^{n}\right\|_{L^{\infty}} \\
\leq & c\left(h \tau+h^{2}\right)+c\left(\tau^{\frac{1}{2}}+h\right)
\end{aligned}
$$

(use (5.16) to estimate $\left\|\rho_{\tau}^{n}-P_{h} \rho_{\tau}^{n}\right\|_{L^{2}}$ and (5.82) to estimate $\left\|e_{\rho, h}^{n}\right\|_{L^{2}}$ ) (use (3.9) to estimate $\left\|\mathbf{u}_{\tau}^{n}\right\|_{L^{\infty}}$ and $\left\|\nabla \mathbf{u}_{\tau}^{n}\right\|_{L^{\infty}}$ )

$$
\leq c\left(\tau^{\frac{1}{2}}+h\right)
$$


Since

$$
\begin{aligned}
B\left(\left(\mathbf{e}_{\mathbf{u}, h}^{n}, e_{p, h}^{n}\right),\left(D_{\tau} \mathbf{e}_{\mathbf{u}, h}^{n}, 0\right)\right) & =\left(\nabla \mathbf{e}_{\mathbf{u}, h}^{n}, \nabla D_{\tau} \mathbf{e}_{\mathbf{u}, h}^{n}\right)-\left(e_{p, h}^{n}, \nabla \cdot D_{\tau} \mathbf{e}_{\mathbf{u}, h}^{n}\right) \\
& =\left(\nabla \mathbf{e}_{\mathbf{u}, h}^{n}, \nabla D_{\tau} \mathbf{e}_{\mathbf{u}, h}^{n}\right) \\
& \geq \frac{1}{2} D_{\tau}\left\|\nabla \mathbf{e}_{\mathbf{u}, h}^{n}\right\|_{L^{2}}^{2},
\end{aligned}
$$

substituting (5.92)-(5.102) into (5.90) yields

$$
\begin{aligned}
& \left(\rho_{h}^{n-1} D_{\tau} \mathbf{e}_{\mathbf{u}, h}^{n}, D_{\tau} \mathbf{e}_{\mathbf{u}, h}^{n}\right)+\frac{1}{2} D_{\tau}\left\|\nabla \mathbf{e}_{\mathbf{u}, h}^{n}\right\|_{L^{2}}^{2} \\
& \quad \leq \epsilon\left\|D_{\tau} \mathbf{e}_{\mathbf{u}, h}^{n}\right\|_{L^{2}}^{2}+\sum_{j=0}^{10} c \epsilon^{-1}\left\|K_{j}\right\|_{L^{2}}^{2} \\
& \quad \leq \epsilon\left\|D_{\tau} \mathbf{e}_{\mathbf{u}, h}^{n}\right\|_{L^{2}}^{2}+c \epsilon^{-1}\left(\tau+h^{2}\right)+c \epsilon^{-1}\left(\left\|D_{\tau} \mathbf{u}_{\tau}^{n}\right\|_{H^{2}}^{2}+\left\|D_{\tau} p_{\tau}^{n}\right\|_{H^{1}}^{2}\right) h^{4},
\end{aligned}
$$

which further implies (by choosing a sufficient small $\epsilon$ and using (5.32), and summing up the inequalities for $n=1, \ldots, N)$

$$
\begin{aligned}
& \tau \sum_{n=1}^{N}\left\|D_{\tau} \mathbf{e}_{\mathbf{u}, h}^{n}\right\|_{L^{2}}^{2}+\max _{1 \leq n \leq N}\left\|\nabla \mathbf{e}_{\mathbf{u}, h}^{n}\right\|_{L^{2}}^{2} \\
& \quad \leq c\left\|\nabla \mathbf{e}_{\mathbf{u}, h}^{0}\right\|_{L^{2}}^{2}+c\left(\tau+h^{2}\right)+c \tau \sum_{n=1}^{N}\left(\left\|D_{\tau} \mathbf{u}_{\tau}^{n}\right\|_{H^{2}}^{2}+\left\|D_{\tau} p_{\tau}^{n}\right\|_{H^{1}}^{2}\right) h^{4} \\
& \quad \leq c\left(\tau+h^{2}\right) . \quad(\text { use }(5.27) \text { and }(3.10))
\end{aligned}
$$

Finally, we estimate $\left(e_{p, h}^{n}, \nabla \cdot \mathbf{v}_{h}\right)$ by taking $q_{h}=0$ in (5.56). By using the $K_{j}$ defined in (5.90), we have

$$
\begin{aligned}
\left|\left(e_{p, h}^{n}, \nabla \cdot \mathbf{v}_{h}\right)\right| \\
\quad=\left|\left(\rho_{h}^{n-1} D_{\tau} \mathbf{e}_{\mathbf{u}, h}^{n}, \mathbf{v}_{h}\right)+\left(\nabla \mathbf{e}_{\mathbf{u}, h}^{n}, \nabla \mathbf{v}_{h}\right)+\left(\frac{1}{2} D_{\tau} \rho_{\tau}^{n} \mathbf{e}_{\mathbf{u}, h}^{n}, \mathbf{v}_{h}\right)-\sum_{j=1}^{10}\left(K_{j}, \mathbf{v}_{h}\right)\right| \\
\quad \leq\left(\left\|\rho_{h}^{n-1} D_{\tau} \mathbf{e}_{\mathbf{u}, h}^{n}\right\|_{L^{2}}+\left\|\nabla \mathbf{e}_{\mathbf{u}, h}^{n}\right\|_{L^{2}}+\left\|\frac{1}{2} D_{\tau} \rho_{\tau}^{n} \mathbf{e}_{\mathbf{u}, h}^{n}\right\|_{L^{2}}+\sum_{j=1}^{10}\left\|K_{j}\right\|_{L^{2}}\right)\left\|\mathbf{v}_{h}\right\|_{H^{1}} \\
\quad \leq\left(c\left\|D_{\tau} \mathbf{e}_{\mathbf{u}, h}^{n}\right\|_{L^{2}}+\left\|\nabla \mathbf{e}_{\mathbf{u}, h}^{n}\right\|_{L^{2}}+\left\|\frac{1}{2} D_{\tau} \rho_{\tau}^{n} \mathbf{e}_{\mathbf{u}, h}^{n}\right\|_{L^{2}}+\sum_{j=1}^{10}\left\|K_{j}\right\|_{L^{2}}\right)\left\|\nabla \mathbf{v}_{h}\right\|_{L^{2}} \\
\quad \leq\left(c\left\|D_{\tau} \mathbf{e}_{\mathbf{u}, h}^{n}\right\|_{L^{2}}+\left\|\nabla \mathbf{e}_{\mathbf{u}, h}^{n}\right\|_{L^{2}}+c\left(\tau^{\frac{1}{2}}+h\right)+c h^{2}\left(\left\|D_{\tau} \mathbf{u}_{\tau}^{n}\right\|_{H^{2}}+\left\|D_{\tau} p_{\tau}^{n}\right\|_{H^{1}}\right)\right)\left\|\nabla \mathbf{v}_{h}\right\|_{L^{2}},
\end{aligned}
$$

where we have used (5.92)-(5.102) in the last inequality. The last inequality, together with the inf-sup condition (2.9), implies

$$
\left\|e_{p, h}^{n}\right\|_{L^{2}} \leq c\left(\left\|D_{\tau} \mathbf{e}_{\mathbf{u}, h}^{n}\right\|_{L^{2}}+\left\|\nabla \mathbf{e}_{\mathbf{u}, h}^{n}\right\|_{L^{2}}\right)+c\left(\tau^{\frac{1}{2}}+h\right)+c h^{2}\left(\left\|D_{\tau} \mathbf{u}_{\tau}^{n}\right\|_{H^{2}}+\left\|D_{\tau} p_{\tau}^{n}\right\|_{H^{1}}\right) .
$$

By summing up the inequality above for $n=1, \ldots, N$, and using (5.104) and (3.10), we have

$$
\sum_{n=1}^{N} \tau\left\|e_{p, h}^{n}\right\|_{L^{2}}^{2} \leq c\left(\tau+h^{2}\right) .
$$

To summarize, (5.22) has been proved at the end of Section 5.6, and (5.82) and (5.105) imply (5.23). The proof of Proposition 3.2 is completed. 
TABLE 1. Rates of convergence and error for different mesh size $h$ with $\tau=h^{2}$.

\begin{tabular}{lllllll}
\hline \hline$h$ & $\frac{\left\|\rho^{N}-\rho_{h}^{N}\right\|_{L^{2}}}{\left\|\rho^{N}\right\|_{L^{2}}}$ & Order & $\frac{\left\|u^{N}-u_{h}^{N}\right\|_{L^{2}}}{\left\|u^{N}\right\|_{L^{2}}}$ & Order & $\frac{\left\|p^{N}-p_{h}^{N}\right\|_{L^{2}}}{\left\|p^{N}\right\|_{L^{2}}}$ & Order \\
\hline $1 / 8$ & $4.870 \mathrm{E}-04$ & - & $9.748 \mathrm{E}-03$ & - & $3.72 \mathrm{E}-03$ & - \\
$1 / 16$ & $1.216 \mathrm{E}-04$ & 2.00 & $2.505 \mathrm{E}-03$ & 1.96 & $9.30 \mathrm{E}-04$ & 2.00 \\
$1 / 32$ & $3.039 \mathrm{E}-05$ & 2.00 & $6.285 \mathrm{E}-04$ & 1.99 & $2.33 \mathrm{E}-04$ & 1.99 \\
$1 / 64$ & $7.595 \mathrm{E}-06$ & 2.00 & $1.598 \mathrm{E}-04$ & 1.97 & $5.94 \mathrm{E}-05$ & 1.97 \\
\hline
\end{tabular}

TABLE 2. Rates of convergence and error in time.

\begin{tabular}{lllllll}
\hline \hline$\tau$ & $\frac{\left\|\rho^{N}-\rho_{h}^{N}\right\|_{L^{2}}}{\left\|\rho^{N}\right\|_{L^{2}}}$ & Order & $\frac{\left\|u^{N}-u_{h}^{N}\right\|_{L^{2}}}{\left\|u^{N}\right\|_{L^{2}}}$ & Order & $\frac{\left\|p^{N}-p_{h}^{N}\right\|_{L^{2}}}{\left\|p^{N}\right\|_{L^{2}}}$ & Order \\
\hline 0.1 & $5.931 \mathrm{E}-03$ & - & $2.940 \mathrm{E}-02$ & - & $3.436 \mathrm{E}-02$ & - \\
$2^{-1} \times 0.1$ & $2.983 \mathrm{E}-03$ & 0.99 & $1.477 \mathrm{E}-02$ & 0.99 & $1.750 \mathrm{E}-02$ & 0.97 \\
$2^{-2} \times 0.1$ & $1.496 \mathrm{E}-03$ & 0.99 & $7.403 \mathrm{E}-03$ & 0.99 & $8.834 \mathrm{E}-03$ & 0.98 \\
$2^{-3} \times 0.1$ & $7.492 \mathrm{E}-04$ & 0.99 & $3.707 \mathrm{E}-03$ & 0.99 & $4.438 \mathrm{E}-03$ & 0.99 \\
$2^{-4} \times 0.1$ & $3.747 \mathrm{E}-04$ & 0.99 & $1.855 \mathrm{E}-03$ & 0.99 & $2.225 \mathrm{E}-03$ & 0.99 \\
$2^{-5} \times 0.1$ & $1.872 \mathrm{E}-04$ & 1.00 & $9.273 \mathrm{E}-04$ & 0.99 & $1.114 \mathrm{E}-03$ & 0.99 \\
\hline
\end{tabular}

\section{Numerical RESUlts}

In this section, we present numerical tests to support the theoretical result proved in Theorem 2.2. To this end, we solve the equations

$$
\begin{aligned}
\partial_{t} \rho+\nabla \cdot(\rho \mathbf{u}) & =f, \\
\rho \partial_{t} \mathbf{u}+\rho \mathbf{u} \cdot \nabla \mathbf{u}+\nabla p-\mu \Delta \mathbf{u} & =\mathbf{g}, \\
\nabla \cdot \mathbf{u} & =0,
\end{aligned}
$$

with $\mu=0.001$, in the unit square $\Omega=[0,1] \times[0,1]$ by the proposed method up to time $T=0.5$, where $f$ and $\mathrm{g}$ are determined by substituting the following exact solution into the equations (6.1)-(6.3):

$$
\begin{aligned}
& \rho(x, y, t)=2+x(x-1) \cos (\sin (t))+y(y-1) \sin (\sin (t)) \\
& u(x, y, t)=\left(\begin{array}{c}
t^{3} y^{2}(y-1) \\
t^{2} x^{2}(x-1)
\end{array}\right) \\
& p(x, y, t)=t x+y-(t+1) / 2 .
\end{aligned}
$$

To illustrate the spatial order of convergence, we present the errors of the numerical solutions in Table 1 for different mesh size $h$ with $\tau=h^{2}$, where we see that the numerical solutions has second-order convergence. To illustrate the temporal order of convergence, the computations are done for different step sizes $\tau$, with a fixed sufficiently small mesh size $h$ such that the error from the spatial discretization is negligible in observing the temporal order of convergence. The numerical results are presented in Table 2, where we see that the temporal order of convergence is also consistent with the error estimate presented in Theorem 2.2. 


\section{Appendix A. Existence And uniqueness of solutions for (3.1)-(3.4)}

We assume that $\rho_{\tau}^{n} \in H^{2}(\Omega) \cap L^{\infty}(\Omega)$ and $\mathbf{u}_{\tau}^{n} \in \mathbf{W}^{1, \infty}(\Omega) \cap \mathbf{H}^{2}(\Omega) \cap \mathbf{H}_{0}^{1}(\Omega)$ are given for $n=0, \ldots, m-1$, such that $\nabla \cdot \mathbf{u}_{\tau}^{n}=0$ and (4.18), (4.19) hold. Under this assumption, we prove the existence and uniqueness of the solution $\left(\rho_{\tau}^{m}, \mathbf{u}_{\tau}^{m}, p_{\tau}^{m}\right)$.

\section{Part I: Well-posedness of (3.1)}

For the given $\rho_{\tau}^{m-1} \in H^{2}(\Omega) \cap L^{\infty}(\Omega)$ and $\mathbf{u}_{\tau}^{m-1} \in \mathbf{W}^{1, \infty}(\Omega) \cap \mathbf{H}^{2}(\Omega) \cap \mathbf{H}_{0}^{1}(\Omega)$, with $\nabla \cdot \mathbf{u}_{\tau}^{m-1}=0$, we can extend $\rho_{\tau}^{m-1}$ to $\widetilde{\rho}_{\tau}^{m-1} \in H^{1}\left(\mathbb{R}^{2}\right)$ such that $\widetilde{\rho}_{\tau}^{m-1}=\rho_{\tau}^{m-1}$ in $\Omega$ and the following estimates hold (cf. [50], Chap. VI, Thm. 5, pp. 181):

$$
\begin{aligned}
\left\|\widetilde{\rho}_{\tau}^{m-1}\right\|_{L^{2}\left(\mathbb{R}^{2}\right)} & \leq c\left\|\rho_{\tau}^{m-1}\right\|_{L^{2}(\Omega)}, \\
\left\|\widetilde{\rho}_{\tau}^{m-1}\right\|_{H^{1}\left(\mathbb{R}^{2}\right)} & \leq c\left\|\rho_{\tau}^{m-1}\right\|_{H^{1}(\Omega)} .
\end{aligned}
$$

The truncated function

$$
\bar{\rho}_{\tau}^{m-1}(x)=\min \left(\max \left(\min _{y \in \bar{\Omega}} \rho_{\tau}^{m-1}(y), \widetilde{\rho}_{\tau}^{m-1}(x)\right), \max _{y \in \bar{\Omega}} \rho_{\tau}^{m-1}(y)\right), \quad x \in \mathbb{R}^{2},
$$

satisfies $\bar{\rho}_{\tau}^{m-1} \in H^{1}\left(\mathbb{R}^{2}\right)$ and

$$
\min _{y \in \bar{\Omega}} \rho_{\tau}^{m-1}(y) \leq \bar{\rho}_{\tau}^{m-1}(x) \leq \max _{y \in \bar{\Omega}} \rho_{\tau}^{m-1}(y), \quad \forall x \in \mathbb{R}^{2} .
$$

Similarly, the function

$$
\widetilde{\mathbf{u}}_{\tau}^{m-1}(x):= \begin{cases}\mathbf{u}_{\tau}^{m-1}(x) & x \in \Omega \\ 0 & x \in \mathbb{R}^{2} \backslash \Omega,\end{cases}
$$

extends $\mathbf{u}_{\tau}^{m-1} \in \mathbf{W}^{1, \infty}(\Omega) \cap \mathbf{H}_{0}^{1}(\Omega)$ to $\widetilde{\mathbf{u}}_{\tau}^{m-1} \in \mathbf{W}^{1, \infty}\left(\mathbb{R}^{2}\right)$.

With the extensions above, we define the bilinear form

$$
a(\phi, \varphi):=\int_{\mathbb{R}^{2}}\left(\tau^{-1} \phi+\widetilde{\mathbf{u}}_{\tau}^{m-1} \cdot \nabla \phi\right) \varphi \mathrm{d} x+\int_{\mathbb{R}^{2}} \epsilon \nabla \phi \cdot \nabla \varphi \mathrm{d} x, \quad \forall \phi, \varphi \in H^{1}\left(\mathbb{R}^{2}\right),
$$

which is coercive on $H^{1}\left(\mathbb{R}^{2}\right)$ for any fixed $\epsilon \in(0,1)$, i.e.,

$$
a(\phi, \phi)=\tau^{-1}\|\phi\|_{L^{2}\left(\mathbb{R}^{2}\right)}^{2}+\epsilon\|\nabla \phi\|_{L^{2}\left(\mathbb{R}^{2}\right)}^{2} \geq c \epsilon\|\phi\|_{H^{1}\left(\mathbb{R}^{2}\right)}^{2} .
$$

Consequently, the Lax-Milgram lemma implies that the equation

$$
a\left(\rho_{\tau, \epsilon}^{m}, \varphi\right)=\int_{\mathbb{R}^{2}} \tau^{-1} \bar{\rho}_{\tau}^{m-1} \varphi \mathrm{d} x, \quad \forall \varphi \in H^{1}\left(\mathbb{R}^{2}\right),
$$

has a unique solution $\rho_{\tau, \epsilon}^{m} \in H^{1}\left(\mathbb{R}^{2}\right)$. In other words, the second-order elliptic equation

$$
\tau^{-1} \rho_{\tau, \epsilon}^{m}+\widetilde{\mathbf{u}}_{\tau}^{m-1} \cdot \nabla \rho_{\tau, \epsilon}^{m}-\epsilon \Delta \rho_{\tau, \epsilon}^{m}=\tau^{-1} \bar{\rho}_{\tau}^{m-1} \quad \text { in } \quad \mathbb{R}^{2}
$$

has a unique weak solution $\rho_{\tau, \epsilon}^{m} \in H^{1}\left(\mathbb{R}^{2}\right)$. The equation above also implies

$$
\epsilon \Delta \rho_{\tau, \epsilon}^{m}=\tau^{-1} \rho_{\tau, \epsilon}^{m}+\widetilde{\mathbf{u}}_{\tau}^{m-1} \cdot \nabla \rho_{\tau, \epsilon}^{m}-\tau^{-1} \bar{\rho}_{\tau}^{m-1} \in L^{2}\left(\mathbb{R}^{2}\right) .
$$

Then the elliptic regularity (cf. [22], Thm. 3.2.1.2) further implies $\rho_{\tau, \epsilon}^{m} \in H^{2}\left(\mathbb{R}^{2}\right)$. 
Integrating (A.7) against the test function $\varphi=\max \left(0, \rho_{\tau, \epsilon}^{m}-\max _{y \in \bar{\Omega}} \bar{\rho}_{\tau}^{m-1}(y)\right)$ yields

$$
\tau^{-1}\|\varphi\|_{L^{2}\left(\mathbb{R}^{2}\right)}^{2}+\epsilon\|\nabla \varphi\|_{L^{2}\left(\mathbb{R}^{2}\right)}^{2}=0,
$$

which implies $\varphi=0$. In other words, $\rho_{\tau, \epsilon}^{m}-\max _{y \in \bar{\Omega}} \bar{\rho}_{\tau}^{m-1}(y) \leq 0$. Similarly, Integrating (A.7) against the test function $\varphi=\min \left(0, \rho_{\tau, \epsilon}^{m}-\min _{y \in \bar{\Omega}} \bar{\rho}_{\tau}^{m-1}(y)\right)$ yields $\rho_{\tau, \epsilon}^{m}-\min _{y \in \bar{\Omega}} \bar{\rho}_{\tau}^{m-1}(y) \geq 0$. This proves that the weak solution of (A.7) obeys the maximum principle:

$$
\min _{y \in \mathbb{R}^{2}} \bar{\rho}_{\tau}^{m-1}(y) \leq \rho_{\tau, \epsilon}^{m}(x) \leq \max _{y \in \mathbb{R}^{2}} \bar{\rho}_{\tau}^{m-1}(y), \quad \forall x \in \mathbb{R}^{2} .
$$

Integrating (A.7) against $\rho_{\tau, \epsilon}^{n}$ yields

$$
\left\|\rho_{\tau, \epsilon}^{m}\right\|_{L^{2}\left(\mathbb{R}^{2}\right)} \leq\left\|\bar{\rho}_{\tau}^{m-1}\right\|_{L^{2}\left(\mathbb{R}^{2}\right)} \leq c\left\|\bar{\rho}_{\tau}^{m-1}\right\|_{L^{2}(\Omega)},
$$

where the last inequality is due to (A.1). By differentiating (A.7) with respect to $x_{j}$, we see that

$$
\begin{array}{r}
\tau^{-1} \partial_{x_{j}} \rho_{\tau, \epsilon}^{m}+\widetilde{\mathbf{u}}_{\tau}^{m-1} \cdot \nabla \partial_{x_{j}} \rho_{\tau, \epsilon}^{m}-\epsilon \Delta \partial_{x_{j}} \rho_{\tau, \epsilon}^{m} \\
=-\partial_{x_{j}} \widetilde{\mathbf{u}}_{\tau}^{m-1} \cdot \nabla \rho_{\tau, \epsilon}^{m}+\tau^{-1} \partial_{x_{j}} \bar{\rho}_{\tau}^{m-1} .
\end{array}
$$

Integrating (A.11) against $\partial_{x_{j}} \rho_{\tau, \epsilon}^{m}$ yields

$$
\begin{aligned}
\sum_{j=1}^{2} \tau^{-1}\left\|\partial_{x_{j}} \rho_{\tau, \epsilon}^{m}\right\|_{L^{2}\left(\mathbb{R}^{2}\right)}^{2} & \leq \sum_{j=1}^{2}\left\|\partial_{x_{j}} \widetilde{\mathbf{u}}_{\tau}^{m-1}\right\|_{L^{\infty}\left(\mathbb{R}^{2}\right)}^{2}\left\|\nabla \rho_{\tau, \epsilon}^{m}\right\|_{L^{2}\left(\mathbb{R}^{2}\right)}^{2}+\sum_{j=1}^{2} \tau^{-1}\left\|\partial_{x_{j}} \rho_{\tau}^{m-1}\right\|_{L^{2}\left(\mathbb{R}^{2}\right)}^{2} \\
& \leq \sum_{j=1}^{2}\left\|\partial_{x_{j}} \mathbf{u}_{\tau}^{m-1}\right\|_{L^{\infty}(\Omega)}^{2}\left\|\nabla \rho_{\tau, \epsilon}^{m}\right\|_{L^{2}\left(\mathbb{R}^{2}\right)}^{2}+c \sum_{j=1}^{2} \tau^{-1}\left\|\partial_{x_{j}} \bar{\rho}_{\tau}^{m-1}\right\|_{L^{2}(\Omega)}^{2}
\end{aligned}
$$

where the last inequality is due to (A.2) and (A.3). For sufficiently small $\tau$, i.e.,

$$
\tau \leq \frac{1}{2\left\|\nabla \mathbf{u}_{\tau}^{m-1}\right\|_{L^{\infty}(\Omega)}^{2}},
$$

the first term on the right-hand side of (A.12) can be absorbed by the left-hand side. Consequently, we obtain

$$
\left\|\nabla \rho_{\tau, \epsilon}^{m}\right\|_{L^{2}(\Omega)}^{2} \leq c\left\|\nabla \rho_{\tau}^{m-1}\right\|_{L^{2}(\Omega)}^{2} .
$$

Since the estimates (A.10) and (A.13) are independent of $\epsilon$, there exists a sequence $\epsilon_{i} \rightarrow 0$ such that $\rho_{\tau, \epsilon_{i}}^{m}$ converges strongly in $L^{2}(B)$ and weakly in $H^{1}(B)$, for any bounded domain $B \subset \mathbb{R}^{2}$. The limit function $\rho_{\tau}^{m}=\lim _{\epsilon_{i} \rightarrow 0} \rho_{\tau, \epsilon_{i}}^{m}$ would satisfy

$$
\int_{\mathbb{R}^{2}}\left(\tau^{-1} \rho_{\tau}^{m}+\widetilde{\mathbf{u}}_{\tau}^{m-1} \cdot \nabla \rho_{\tau}^{m}\right) \varphi \mathrm{d} x=\int_{\mathbb{R}^{2}} \tau^{-1} \rho_{\tau}^{m-1} \varphi \mathrm{d} x, \quad \forall \varphi \in C_{0}^{\infty}\left(\mathbb{R}^{2}\right) .
$$

Since both $\tau^{-1} \rho_{\tau}^{m}+\widetilde{\mathbf{u}}_{\tau}^{m-1} \cdot \nabla \rho_{\tau}^{m}$ and $\tau^{-1} \rho_{\tau}^{m-1}$ are in $L^{2}(\Omega)$ and $C_{0}^{\infty}(\Omega) \subset C_{0}^{\infty}\left(\mathbb{R}^{2}\right)$ is dense in $L^{2}(\Omega)$, it follows that

$$
\int_{\Omega}\left(\tau^{-1} \rho_{\tau}^{m}+\mathbf{u}_{\tau}^{m-1} \cdot \nabla \rho_{\tau}^{m}\right) \varphi \mathrm{d} x=\int_{\Omega} \tau^{-1} \rho_{\tau}^{m-1} \varphi \mathrm{d} x, \quad \forall \varphi \in L^{2}(\Omega)
$$


This proves the existence of a strong solution $\rho_{\tau}^{m} \in H^{1}(\Omega) \cap L^{\infty}(\Omega)$ of (3.1), obeying the maximum principle (in view of (A.4) and (A.9)):

$$
\min _{y \in \bar{\Omega}} \rho_{\tau}^{m-1}(y) \leq \rho_{\tau}^{m}(x) \leq \max _{y \in \bar{\Omega}} \rho_{\tau}^{m-1}(y), \quad \forall x \in \Omega .
$$

If there exist two such solutions $\rho_{\tau}^{m}, \widetilde{\rho}_{\tau}^{m} \in H^{1}(\Omega)$ for the equation (3.1), then we have

$$
\tau^{-1}\left(\rho_{\tau}^{m}-\widetilde{\rho}_{\tau}^{m}\right)+\mathbf{u}_{\tau}^{m-1} \cdot \nabla\left(\rho_{\tau}^{m}-\widetilde{\rho}_{\tau}^{m}\right)=0 .
$$

Integrating the above equation against $\rho_{\tau}^{m}-\widetilde{\rho}_{\tau}^{m}$ and using the divergence-free property of $\mathbf{u}_{\tau}^{m-1}$, we immediately obtain

$$
\tau^{-1}\left\|\rho_{\tau}^{m}-\widetilde{\rho}_{\tau}^{m}\right\|_{L^{2}}^{2}=0
$$

which implies $\rho_{\tau}^{m}=\widetilde{\rho}_{\tau}^{m}$. The uniqueness is proved.

\section{Part II: Well-posedness of (3.2) and (3.3)}

For the given $\rho_{\tau}^{m-1}, \rho_{\tau}^{m} \in H^{2}(\Omega) \cap L^{\infty}(\Omega)$ and $\mathbf{u}_{\tau}^{m-1} \in \mathbf{W}^{1, \infty}(\Omega) \cap \mathbf{H}^{2}(\Omega) \cap \mathbf{H}_{0}^{1}(\Omega)$, with $\rho_{\tau}^{m-1} \geq 0$ and $\rho_{\tau}^{m} \geq 0$ (proved in Part I), we define the bilinear form

$$
\begin{aligned}
b(\mathbf{w}, \mathbf{v}):= & \frac{1}{2} \int_{\Omega}\left(\tau^{-1}\left(\rho_{\tau}^{m}-\rho_{\tau}^{m-1}\right)+\mathbf{u}_{\tau}^{m-1} \cdot \nabla \rho_{\tau}^{m}\right) \mathbf{w} \cdot \mathbf{v} \mathrm{d} x \\
& +\int_{\Omega}\left(\tau^{-1} \rho_{\tau}^{m-1} \mathbf{w}+\rho_{\tau}^{n} \mathbf{u}_{\tau}^{m-1} \cdot \nabla \mathbf{w}\right) \cdot \mathbf{v} \mathrm{d} x \\
& +\mu \int_{\Omega} \nabla \mathbf{w} \cdot \nabla \mathbf{v} \mathrm{d} x, \quad \forall \mathbf{w}, \mathbf{v} \in \stackrel{\mathbf{H}}{\mathrm{div}}^{1}(\Omega)
\end{aligned}
$$

where $\stackrel{\circ}{\mathbf{H}}_{\text {div }}^{1}(\Omega)=\left\{\mathbf{v} \in \mathbf{H}_{0}^{1}(\Omega): \nabla \cdot \mathbf{v}=0\right\}$. It is easy to see that the bilinear form $b(\cdot, \cdot)$ is coercive on $\stackrel{\circ}{\mathbf{H}}_{\text {div }}^{1}(\Omega)$, i.e.,

$$
b(\mathbf{w}, \mathbf{w})=\frac{1}{2} \int_{\Omega} \tau^{-1}\left(\rho_{\tau}^{m}+\rho_{\tau}^{m-1}\right)|\mathbf{w}|^{2} \mathrm{~d} x+\mu \int_{\Omega}|\nabla \mathbf{w}|^{2} \mathrm{~d} x \geq c\|\mathbf{w}\|_{H^{1}}^{2} .
$$

Consequently, the Lax-Milgram lemma implies that the equation

$$
b\left(\mathbf{u}_{\tau}^{m}, \mathbf{v}\right):=\int_{\Omega} \tau^{-1} \rho_{\tau}^{m-1} \mathbf{u}_{\tau}^{m-1} \cdot \mathbf{v} \mathrm{d} x, \quad \forall \mathbf{v} \in \stackrel{\circ}{\mathbf{H}}_{\mathrm{div}}^{1}(\Omega),
$$

has a unique solution $\mathbf{u}_{\tau}^{m} \in \stackrel{\circ}{\mathrm{div}}_{\mathrm{div}}^{1}(\Omega)$, satisfying

$$
\begin{gathered}
\int_{\Omega}\left(\tau^{-1} \rho_{\tau}^{m-1} \mathbf{u}_{\tau}^{m}+\rho_{\tau}^{m} \mathbf{u}_{\tau}^{m-1} \cdot \nabla \mathbf{u}_{\tau}^{m}\right) \cdot \mathbf{v} \mathrm{d} x+\mu \int_{\Omega} \nabla \mathbf{u}_{\tau}^{m} \cdot \nabla \mathbf{v} \mathrm{d} x \\
=\int_{\Omega} \tau^{-1} \rho_{\tau}^{m-1} \mathbf{u}_{\tau}^{m-1} \cdot \mathbf{v} \mathrm{d} x, \quad \forall \mathbf{v} \in \stackrel{\mathrm{H}}{\mathrm{div}}^{1}(\Omega)
\end{gathered}
$$

By using the notation of Section 4.1, the last equation implies, for $\mathbf{v} \in \mathbf{C}_{0}^{\infty}(\Omega)$,

$$
\begin{aligned}
-\left(\mu \Delta \mathbf{u}_{\tau}^{m}, \mathbf{v}\right) & =-\left(\mu \mathbf{u}_{\tau}^{m}, \Delta \mathbf{v}\right) \\
& =-\left(\mu \mathbf{u}_{\tau}^{m}, \mathbf{P}_{\mathrm{div}} \Delta \mathbf{v}\right) \\
& =-\left(\mu \mathbf{u}_{\tau}^{m}, \Delta \mathbf{P}_{\operatorname{div}} \mathbf{v}\right) \\
& =\left(\mu \nabla \mathbf{u}_{\tau}^{m}, \nabla \mathbf{P}_{\operatorname{div}} \mathbf{v}\right)
\end{aligned}
$$




$$
\begin{aligned}
& =\int_{\Omega} \tau^{-1} \rho_{\tau}^{m-1} \mathbf{u}_{\tau}^{m-1} \cdot \mathbf{P}_{\mathrm{div}} \mathbf{v} \mathrm{d} x-\int_{\Omega}\left(\tau^{-1} \rho_{\tau}^{m-1} \mathbf{u}_{\tau}^{m}+\rho_{\tau}^{m} \mathbf{u}_{\tau}^{m-1} \cdot \nabla \mathbf{u}_{\tau}^{m}\right) \cdot \mathbf{P}_{\operatorname{div}} \mathbf{v} \mathrm{d} x \\
& =\int_{\Omega} \mathbf{P}_{\operatorname{div}}\left(\tau^{-1} \rho_{\tau}^{m-1} \mathbf{u}_{\tau}^{m-1}-\tau^{-1} \rho_{\tau}^{m-1} \mathbf{u}_{\tau}^{m}-\rho_{\tau}^{m} \mathbf{u}_{\tau}^{m-1} \cdot \nabla \mathbf{u}_{\tau}^{m}\right) \mathbf{v} \mathrm{d} x,
\end{aligned}
$$

where the last equality is due to the self-adjointness of the projection operator $\mathbf{P}_{\text {div }}$. This last equality implies he following equations:

$$
\begin{aligned}
-\mu \Delta \mathbf{u}_{\tau}^{m}+\nabla \phi & =\tau^{-1} \rho_{\tau}^{m-1} \mathbf{u}_{\tau}^{m-1}-\tau^{-1} \rho_{\tau}^{m-1} \mathbf{u}_{\tau}^{m}-\rho_{\tau}^{m} \mathbf{u}_{\tau}^{m-1} \cdot \nabla \mathbf{u}_{\tau}^{m} \in \mathbf{L}^{2}(\Omega), \\
\nabla \cdot \mathbf{u}_{\tau}^{m} & =0 .
\end{aligned}
$$

The standard $H^{2}$ estimate for the Stokes equations (cf. [29]) implies $\mathbf{u}_{\tau}^{m} \in \mathbf{H}^{2}(\Omega) \cap \mathbf{H}_{0}^{1}(\Omega)$, and (A.22) implies

$$
\mathbf{P}_{\text {div }}\left(\rho_{\tau}^{m-1} D_{\tau} \mathbf{u}_{\tau}^{m}+\rho_{\tau}^{m} \mathbf{u}_{\tau}^{m-1} \cdot \nabla \mathbf{u}_{\tau}^{m}-\mu \Delta \mathbf{u}_{\tau}^{m}\right)=0 .
$$

By the Helmoholtz-Weyl decomposition (4.4), there exists a unique $p_{\tau}^{m} \in H^{1}(\Omega)$ such that $\int_{\Omega} p_{\tau}^{m} \mathrm{~d} x=0$ and

$$
\rho_{\tau}^{m-1} D_{\tau} \mathbf{u}_{\tau}^{m}+\rho_{\tau}^{m} \mathbf{u}_{\tau}^{m-1} \cdot \nabla \mathbf{u}_{\tau}^{m}-\mu \Delta \mathbf{u}_{\tau}^{m}=-\nabla p_{\tau}^{m} .
$$

This proves the existence and uniqueness of the solution

$$
\left(\mathbf{u}_{\tau}^{m}, p_{\tau}^{m}\right) \in\left(\mathbf{H}^{2}(\Omega) \cap \mathbf{H}_{0}^{1}(\Omega)\right) \times\left(H^{1}(\Omega) \cap L_{0}^{2}(\Omega)\right)
$$

for (3.2) and (3.3) with $n=m$.

Acknowledgements. The work of Wentao Cai was partially supported by NSFC (project no. 11901142) and The Hong Kong Polytechnic University (project no. 1-ZE6L). The work of Buyang Li was partially supported by a Hong Kong RGC grant (project no. 15301818). The work of Ying Li was partially supported by the National key R\&D program of China (project no. 2017YFB0202103).

\section{REFERENCES}

[1] Y. Achdou and J.-L. Guermond, Convergence analysis of a finite element projection/Lagrange-Galerkin method for the incompressible Navier-Stokes equations. SIAM J. Numer. Anal. 37 (2000) 799-826.

[2] R. Adams and J. Fournier, Sobolev Spaces. In: Pure and Applied Mathematics. Elsevier Science (2003).

[3] G. Akrivis and B. Li, Maximum norm analysis of implicit-explicit backward difference formulas for nonlinear parabolic equations. IMA J. Numer. Anal. 38 (2018) 75-101.

[4] G. Akrivis, B. Li and C. Lubich, Combining maximal regularity and energy estimates for time discretizations of quasilinear parabolic equations. Math. Comput. 86 (2017) 1527-1552.

[5] A.S. Almgren, J.B. Bell, P. Colella, L.H. Howell and M.L. Welcome, A conservative adaptive projection method for the variable density incompressible Navier-Stokes equations. J. Comput. Phys. 142 (1998) 1-46.

[6] A. Ashyralyev, S. Piskarev and L. Weis, On well-posedness of difference schemes for abstract parabolic equations in $L_{p}([0, T] ; E)$ spaces. Numer. Funct. Anal. Optim. 23 (2002) 669-693.

[7] J.B. Bell and D.L. Marcus, A second-order projection method for variable-density flows. J. Comput. Phys. 101 (1992) 334-348.

[8] D. Boffi, Stability of higher order triangular Hood-Taylor methods for the stationary stokes equations. Math. Models Methods Appl. Sci. 04 (1994) 223-235.

[9] S.C. Brenner and R. Scott, The Mathematical Theory of Finite Element Methods, 3rd ed. In: Texts in Applied Mathematics. Springer-Verlag New York (2008).

[10] S. Brenner, J. Cui, Z. Nan and L. Sung, Hodge decomposition for divergence-free vector fields and two-dimensional Maxwell's equations. Math. Comput. 81 (2012) 643-659.

[11] C. Calgaro, E. Creusé and T. Goudon, An hybrid finite volume-finite element method for variable density incompressible flows. J. Comput. Phys. 227 (2008) 4671-4696.

[12] A.J. Chorin, Numerical solution of the Navier-Stokes equations. Math. Comput. 22 (1968) 745-762.

[13] M. Crouzeix and V. Thomée, The stability in $L_{p}$ and $W_{p}^{1}$ of the $L_{2}$-projection onto finite element function spaces. Math. Comput. 48 (1987) 521-532.

[14] R. Danchin, Density-dependent incompressible fluids in bounded domains. J. Math. Fluid Mech. 8 (2006) 333-381. 
[15] D.A. Di Pietro and A. Ern, Mathematical Aspects of Discontinuous Galerkin Methods. In: Vol. 69 of Mathématiques et Applications. Springer Berlin Heidelberg (2012).

[16] G.P. Galdi, An Introduction to the Mathematical Theory of the Navier-Stokes Equations. In: Springer Monographs in Mathematics. Springer-Verlag New York (2011).

[17] H. Gao, B. Li and W. Sun, Stability and convergence of fully discrete Galerkin FEMs for the nonlinear thermistor equations in a nonconvex polygon. Numer. Math. 136 (2017) 383-409.

[18] M. Geissert, Discrete maximal $L^{p}$ regularity for finite element operators. SIAM J. Numer. Anal. 44 (2006) 677-698.

[19] J. Geng and Z. Shen, The Neumann problem and Helmholtz decomposition in convex domains. J. Funct. Anal. 259 (2010) 2147-2164.

[20] V. Girault and P.A. Raviart, Finite Element Methods for Navier-Stokes Equations: Theory and Algorithms. In: Springer Series in Computational Mathematics. Springer Berlin Heidelberg (1986).

[21] V. Girault, R. Nochetto and R. Scott, Maximum-norm stability of the finite element Stokes projection. J. Math. Pures Appl. 84 (2005) 279-330.

[22] P. Grisvard, Elliptic Problems in Nonsmooth Domains. Society for Industrial and Applied Mathematics (2011).

[23] J.L. Guermond and L. Quartapelle, A projection FEM for variable density incompressible flows. J. Comput. Phys. 165 (2000) $167-188$.

[24] J.L. Guermond and A. Salgado, A fractional step method based on a pressure Poisson equation for incompressible flows with variable density. C. R. Math. 346 (2008) 913-918.

[25] J.L. Guermond and A. Salgado, A splitting method for incompressible flows with variable density based on a pressure Poisson equation. J. Comput. Phys. 228 (2009) 2834-2846.

[26] J.L. Guermond and A. Salgado, Error analysis of a fractional time-stepping technique for incompressible flows with variable density. SIAM J. Numer. Anal. 49 (2011) 917-940.

[27] J. Guermond, P. Minev and J. Shen, An overview of projection methods for incompressible flows. Comput. Methods Appl. Mech. Eng. 195 (2006) 6011-6045.

[28] J.G. Heywood and R. Rannacher, Finite-element approximation of the nonstationary Navier-Stokes problem part IV: error analysis for second-order time discretization. SIAM J. Numer. Anal. 27 (1990) 353-384.

[29] R.B. Kellogg and J.E. Osborn, A regularity for the Stokes problem in a convex polygon. J. Funct. Anal. 21 (1976) $397-431$.

[30] B. Kovács, B. Li and C. Lubich, A-stable time discretizations preserve maximal parabolic regularity. SIAM J. Numer. Anal. 54 (2016) 3600-3624.

[31] P.C. Kunstmann, B. Li and C. Lubich, Runge-Kutta time discretization of nonlinear parabolic equations studied via discrete maximal parabolic regularity. Found. Comput. Math. 18 (2018) 1109-1130.

[32] O. Ladyzhenskaya and V. Solonnikov, Unique solvability of an initial- and boundary-value problem for viscous incompressible inhomogeneous fluids. J. Sov. Math. 9 (1978) 697-749.

[33] B. Li, Maximum-norm stability and maximal $L^{p}$ regularity of FEMs for parabolic equations with Lipschitz continuous coefficients. Numer. Math. 131 (2015) 489-516.

[34] B. Li, Analyticity, maximal regularity and maximum-norm stability of semi-discrete finite element solutions of parabolic equations in nonconvex polyhedra. Math. Comput. 88 (2019) 1-44.

[35] B. Li and W. Sun, Unconditional convergence and optimal error estimates of a Galerkin-mixed FEM for incompressible miscible flow in porous media. SIAM J. Numer. Anal. 51 (2013) 1959-1977.

[36] B. Li and W. Sun, Linearized FE approximations to a nonlinear gradient flow. SIAM J. Numer. Anal. 52 (2014) $2623-2646$.

[37] B. Li and W. Sun, Regularity of the diffusion-dispersion tensor and error analysis of FEMs for a porous media flow. SIAM J. Numer. Anal. 53 (2015) 1418-1437.

[38] B. Li and W. Sun, Maximal $L^{p}$ analysis of finite element solutions for parabolic equations with nonsmooth coefficients in convex polyhedra. Math. Comput. 86 (2017) 1071-1102.

[39] B. Li and Z. Zhang, Mathematical and numerical analysis of time-dependent Ginzburg-Landau equations in nonconvex polygons based on Hodge decomposition. Math. Comput. 86 (2017) 1579-1608.

[40] Y. Li, L. Mei, J. Ge and F. Shi, A new fractional time-stepping method for variable density incompressible flows. J. Comput. Phys. 242 (2013) 124-137.

[41] Y. Li, J. Li, L. Mei and Y. Li, Mixed stabilized finite element methods based on backward difference/Adams-Bashforth scheme for the time-dependent variable density incompressible flows. Comput. Math. App. 70 (2015) 2575-2588.

[42] C. Liu and N.J. Walkington, Convergence of numerical approximations of the incompressible Navier-Stokes equations with variable density and viscosity. SIAM J. Numer. Anal. 45 (2007) 1287-1304.

[43] A. Lunardi, Analytic Semigroups and Optimal Regularity in Parabolic Problems. In: Vol. 16 of Progress in Nonlinear Differential Equations and their Applications. Birkhäuser Verlag (1995).

[44] E. Ortega-Torres, P. Braz e Silva and M. Rojas-Medar, Analysis of an iterative method for variable density incompressible fluids. Ann. Univ. Ferrara 55 (2009) 129.

[45] A. Prohl, Projection and Quasi-Compressibility Methods for Solving the Incompressible Navier-Stokes Equations. In: Advances in Numerical Mathematics. Vieweg+Teubner Verlag (1997).

[46] J.-H. Pyo and J. Shen, Gauge-Uzawa methods for incompressible flows with variable density. J. Comput. Phys. 221 (2007) 181-197.

[47] R. Rannacher, On Chorin's Projection Method for the Incompressible Navier-Stokes Equations. Springer Berlin Heidelberg, Berlin, Heidelberg (1992) 167-183. 
[48] J. Shen, On error estimates of projection methods for Navier-Stokes equations: first-order schemes. SIAM J. Numer. Anal. 29 (1992) 57-77.

[49] J. Shen, On error estimates of the projection methods for the Navier-Stokes equations: second-order schemes. Math. Comput. 65 (1996) 1039-1065.

[50] E.M. Stein, Singular Integrals and Differentiability Properties of Functions. In: Monographs in Harmonic Analysis. Princeton University Press (1970).

[51] L. Tartar, An Introduction to Sobolev Spaces and Interpolation Spaces. In: Vol. 3 of Lecture Notes of the Unione Matematica Italiana. Springer-Verlag Berlin Heidelberg (2007). 